\title{
GAMMA-RAY ANGULAR CORRELATION AND POLARIZATION MEASUREMENTS IN THE REACTION ${ }^{35} \mathrm{Cl}(\mathrm{p}, \gamma)^{36} \mathrm{Ar}$
}

\author{
F. C. ERNÉ \\ Fysisch Laboratorium der Rijksuniversiteit, Utrecht
}

Received 10 March 1966

\begin{tabular}{|c|c|c|c|c|c|}
\hline$E_{\mathrm{x}}(\mathrm{MeV})$ & $J \pi$ & $E_{\mathbf{x}}(\mathrm{MeV})$ & $J^{n}$ & $E_{\mathrm{x}}(\mathrm{MeV})$ & $J^{\pi}$ \\
\hline 1.98 & $2^{+}$ & 5.19 & $5^{-}$ & 9.57 & 4 \\
\hline 4.19 & $3^{-}$ & 5.85 & (1) & 9.59 & $2^{+}$ \\
\hline 4.43 & $4^{+}$ & 5.91 & $\left(4^{-}\right)$ & 9.61 & (0) \\
\hline 4.45 & $2^{(+)}$ & 7.18 & $3^{(+)}$ & 10.22 & $4^{(-)}$ \\
\hline 4.97 & $2^{-}\left(3^{-}\right)$ & 9.22 & $1^{(+)}$ & 10.30 & $4^{+}$ \\
\hline 4.99 & $\left(2^{+}\right)$ & 9.34 & 3 & 11.03 & $3^{+}$ \\
\hline
\end{tabular}

The 4.34 and $5.21 \mathrm{MeV}$ levels are suggested to have $J^{\pi}=0^{+}$.

In addition a number of multipole mixing ratios for proton capture and gamma-ray emission have been determined. Isospin selection rules appear to work effectively in this nucleus. This results in the emission of $\mathrm{E} 2$ and $\mathrm{E} 3$ radiation in several cases. The main configurations of several levels in ${ }^{36} \mathrm{Ar}$ and of some levels in ${ }^{35} \mathrm{Cl}$, excited by proton emission from the capture state, are discussed.

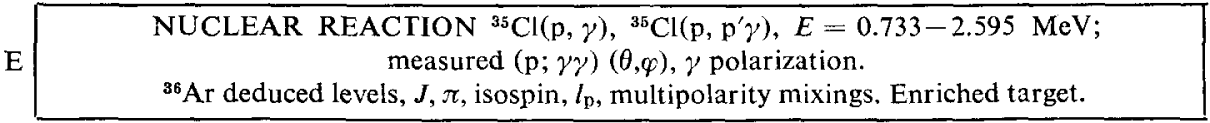

\section{Introduction}

The nucleus ${ }^{36} \mathrm{Ar}$ has more or less escaped the interest of nuclear spectroscopists up to now. Only recently has the level scheme been established ${ }^{\mathbf{1}}$ ) from the reaction ${ }^{39} \mathrm{~K}(\mathrm{p}, \alpha){ }^{36} \mathrm{Ar}$, but for none of the levels below $9 \mathrm{MeV}$ are the spins known with certainty. For some of the higher levels, corresponding to resonances in the reaction ${ }^{32} \mathrm{~S}(\alpha, \gamma){ }^{36} \mathrm{Ar}$, the spin and parity have been determined $\left.{ }^{2}\right)$. Apparently, the nucleus ${ }^{36} \mathrm{Ar}$ holds no key position in simple shell-model considerations, being in the middle of a shell, and not in a deformed region. However, since recent calculations, covering the entire $2 \mathrm{~s}_{\frac{1}{2}}$ and $1 \mathrm{~d}_{\frac{3}{2}}$ shells ${ }^{3}$ ), also predicted properties of a number of ${ }^{36} \mathrm{Ar}$ levels, an experimental verification was desirable. 
The determination of spins and parities of levels in ${ }^{36} \mathrm{Ar}$ is the aim of the present investigation. Double and triple angular correlation measurements of the reaction ${ }^{35} \mathrm{Cl}(\mathrm{p}, \gamma){ }^{36} \mathrm{Ar}$ provide a good means to achieve this. The reaction shows many moderately strong single resonances, of which the decay is known sufficiently well from a previous investigation ${ }^{4}$ ). Many of these resonances show a decay mainly to a small number of lower levels, thus facilitating the investigation of levels in close doublets or triplets.

A number of continuous parameters is required in the description of angular correlation measurements in a reaction as ${ }^{35} \mathrm{Cl}(\mathrm{p}, \gamma){ }^{36} \mathrm{Ar}$. The formation of a resonance proceeds through two possible channel spins, and in quite a few cases orbital momentum mixtures are found ${ }^{5-7}$ ). Furthermore, the radiation multipolarity mixtures in self-conjugate nuclei vary from pure dipole to almost pure quadrupole due to isospin selection rules; octupole admixtures even occur. These factors make the analysis quite involved, but, on the other hand, demonstrate an abundance of obtainable information. The use of appropriate methods of analysis ${ }^{8,9}$ ) and fast computers can lead to definite conclusions concerning spins and multipole mixtures, see refs. ${ }^{5,6,10,11}$ ).

In the present investigation, measurements have been performed at eight resonances, yielding unique spin values of six bound levels and probable values of four others. Additional polarization measurements at three resonances yielded unambiguous parity determinations of five bound levels.

In $(p, \gamma)$ investigations of even nuclei, performed up to now $5,6,10,11)$, mostly gamma cascades were investigated and successfully analysed in which the second gamma transition proceeded to the $0^{+}$ground state. In these cases it is relatively easy to obtain definite conclusions about the spin combinations, as the last transition in the cascade consists of radiation of pure multipolarity, usually higher than dipole. In the present investigation, bound states with high spins are also observed. These levels show at best a weak ground-state transition, decaying rather through two or more step cascades. A large number of double and triple correlations of the types $(\mathrm{p}, \gamma),(\mathrm{p}, \mathrm{x} \gamma),(\mathrm{p}, \gamma \gamma),(\mathrm{p}, \mathrm{x} \gamma \gamma)$ and $(\mathrm{p}, \gamma \mathrm{x} \gamma)$ can be measured in these cases ${ }^{\dagger}$.

The experiments on multiple cascades, however, are beset by extra difficulties. Coincident summing of pulses from several gamma rays in one crystal obscures crossover transitions. The occurrence of several gamma rays with almost equal energies necessitates the analysis of coincidence spectra, instead of the simpler scaler techniques. Furthermore, the multipolarity of the gamma rays usually is mainly dipole. Dipole radiation gives little information on the spins involved. Triple correlation measurements have to be performed with a rather high accuracy.

Special problems also arise in the analysis of multistep cascades, if one wishes to adhere to a rigorous $\chi^{2}$ method to reject solutions, as has been done in the present work. A customary and obvious procedure of analysis is to determine the resonance spin first by triple correlation measurements of cascades through levels with known spins and to derive the spin of an unknown bound level by additional measurements through

+ Here the symbol $x$ denotes unobserved radiation. 
that level. Whenever possible, this procedure has been applied. In a few cases, however, the resonance spin could not be determined so easily, and a simultaneous analysis of all measurements at one resonance was necessary. Existing methods of analysis were adapted for this procedure, and are described in subsect. 2.3.

The theoretical description of the angular correlation process has been evaluated by various authors and may be found in the refs. ${ }^{8,9,12,13}$ ). In subsect. 2.2 a survey will be given of the formulae and tabulations used. A few reformulations and a formula convenient for the description of coincident summing in double correlations will be introduced.

The analysis and the results of the measurements will be described in detail in subsects. 2.3, 2.4 and 3, whereas in sect. 4 the properties of some bound states in ${ }^{36} \mathrm{Ar}$ and ${ }^{35} \mathrm{Cl}$ will be discussed in connection with recent shell-model calculations.

A summary of the main results of this investigation has been published earlier ${ }^{42}$ ).

\section{Double and Triple Correlations}

\subsection{EXPERIMENTAL PROCEDURE}

The apparatus used to investigate $(\mathrm{p}, \gamma)$ reactions has been described previously by Van Rinsvelt and Smith ${ }^{14}$ ). Protons from the Utrecht 3 MV Van de Graaff accelerator pass through a $90^{\circ}$ analysing magnet and a liquid air trap before hitting the water-cooled target. The target consists of $\mathrm{BaCl}_{2}$ enriched to $99.5 \%{ }^{35} \mathrm{Cl}$, evaporated onto a tantalum backing.

For angular correlation measurements targets are used of about $5 \mathrm{keV}$ thickness; targets of less than $1 \mathrm{keV}$ thickness are used to check whether resonances are single.

The gamma rays are detected in cylindrical Nal scintillation assemblies, $10 \mathrm{~cm}$ thick and $10 \mathrm{~cm}$ diam, surrounded by iron shielding. The crystals are mounted on a turntable on which both counters can move in the horizontal plane and one detector can be placed in a vertical position above the target. The spectra are measured with a 400-channel RIDL analyser.

All angular distribution and correlation measurements are performed with the front of the detectors at $10 \mathrm{~cm}$ distance from the target. This distance gives rise to solid angle attenuation factors $Q_{2}$ and $Q_{4}$ in the angular correlations (see subsect. 2.2), with $Q_{2}$ ranging from 0.89 to 0.92 and $Q_{4}$ from 0.72 to 0.76 , dependent on the channel setting and the gamma-ray energy ${ }^{15}$ ).

One detector, placed at a fixed angle $\theta_{1}=\frac{1}{2} \pi$ (see fig. 1) with respect to the proton beam in a horizontal or vertical position $\left(\phi=\pi\right.$ or $\left.\frac{1}{2} \pi\right)$, serves as a radiation monitor, while the second detector is rotated in the horizontal plane. In this way the angular correlations from single resonances remain symmetric with respect to the plane perpendicular to the beam direction and can be analysed as functions of even Legendre polynomials. The radiation in the movable detector is counted only at the forward angles $\theta_{2}=0^{\circ}, 35^{\circ}, 55^{\circ}$ and $90^{\circ}$ with respect to the proton beam direction. In triple correlation measurements coincidences are measured between the two detectors 
(resolving time $\tau=1 \mu \mathrm{s}$ ). These coincidences are usually recorded in the form of coincidence spectra from which, after analysis, the intensities of several lines can be obtained. Four angles are chosen because the 400 analyser channels can be divided into four sections. Angular distributions and triple correlations can thus be measured without too many read-out problems.

Each angular distribution or correlation measurement consists of several runs in the sequence $\theta_{2}=0^{\circ}, 35^{\circ}, 55^{\circ}, 90^{\circ}, 90^{\circ}, 55^{\circ}, 35^{\circ}$ and $0^{\circ}$. At each angle the measurement lasts about $15 \mathrm{~min}$. The coincidence yield for each angle for each run varies typically between 10 and 100 counts and the number of runs between 10 and 20 .

The eccentricity of the target position is measured with the isotropic $7.89 \mathrm{MeV}$ line from the ${ }^{30} \mathrm{Si}(\mathrm{p}, \gamma){ }^{31} \mathrm{P}$ resonance at $E_{\mathrm{p}}=622 \mathrm{keV}$ and more directly with the isotropic $1.22 \mathrm{MeV}$ line from the ${ }^{35} \mathrm{Cl}\left(\mathrm{p}, \mathrm{p}^{\prime} \gamma\right){ }^{35} \mathrm{Cl}$ reaction (mostly at the strong $2841 \mathrm{keV}$

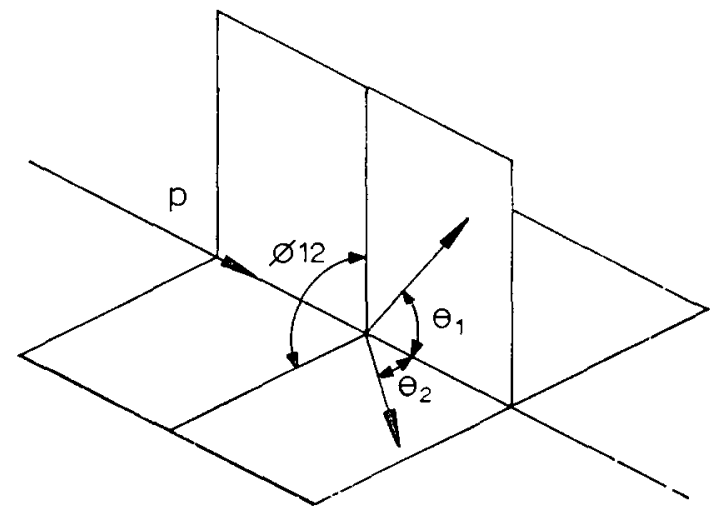

Fig. 1. Definition of the angles for the description of triple correlations.

resonance). The triple correlation measurements of two gamma rays in a cascade, $\gamma_{1}$ and $\gamma_{2}$, are performed in four geometries:

geometry
I: $\theta_{1}=$ variable, $\quad \theta_{2}=\frac{1}{2} \pi$,
$\phi_{12}=\pi$
II: $\theta_{1}=\frac{1}{2} \pi$,
$\theta_{2}=$ variable,
$\phi_{12}=\pi$
$\mathrm{V}: \theta_{1}=$ variable,
$\theta_{2}=\frac{1}{2} \pi$,
$\phi_{12}=\frac{1}{2} \pi$
VI: $\theta_{1}=\frac{1}{2} \pi$,
$\theta_{2}=$ variable,
$\phi_{12}=\frac{1}{2} \pi$

for the meaning of the symbols denoting the angles, see fig. 1 .

In addition angular distributions (double correlations) AD1 and AD2 are measured:

AD1: $\theta_{1}=$ variable, $\quad \gamma_{2}=$ unobserved

AD2: $\gamma_{1}=$ unobserved, $\quad \theta_{2}=$ variable.

These four geometries, together with the two angular distributions, contain most of the information, obtainable from a triple correlation measurement, if radiation parameters of low order are involved in the reaction. To demonstrate this point, the in- 
formation can be conveniently expressed in coefficients $I_{k K M}$ of the series

$$
W\left(\Omega_{\mathrm{p}}, \Omega_{1}, \Omega_{2}\right)=\sum_{k K M} I_{k K M} P_{k K M}\left(\Omega_{\mathrm{p}}, \Omega_{1}, \Omega_{2}\right)
$$

as pointed out by Ferguson ${ }^{13}$ ). The symbols $\Omega_{\mathrm{p}}=\theta_{\mathrm{p}} \phi_{\mathrm{p}}, \Omega_{1}=\theta_{1} \phi_{1}$ and $\Omega_{2}=\theta_{2} \phi_{2}$ denote the directions of the three radiations. The $P_{k K M}$ functions, defined by Biedenharn ${ }^{16}$ ), form an orthonormal set in the space $\Omega_{\mathrm{p}}, \Omega_{1}, \Omega_{2}$. The relation to $X_{K M}^{N}$ functions is given in subsect. 2.2, eq. (25). The indices $k, K$ and $M$ refer to the orders of the radiation parameters of the incoming protons, and of the first and second gamma ray, respectively. They only take even values if the spins and parities of the relevant levels are sharp. If the indices are limited to $k \leqq 8, K \leqq 4$ and $M \leqq 4$, the series contains 18 terms, the $I_{000}$ term excluded. In numerous cases, however, the series terminates before the full 18 terms. In table 1 the maximum number of non-zero terms

TABLE 1

\begin{tabular}{lcc}
$\begin{array}{c}\text { Maximum values of } \\
k K M\end{array}$ & $\begin{array}{c}\text { Number of } \\
\text { relevant } \\
I_{k K M}\end{array}$ & $\begin{array}{c}\text { Number of independent combi- } \\
\text { nations of } I_{k K M} \text { that can be meas- } \\
\text { ured from the geometries I, II, } \\
\text { V, VI and the two angular dis- } \\
\text { tributions }\end{array}$ \\
\hline 844 & 18 & 10 \\
644 & 17 & 10 \\
444 & 14 & 10 \\
442 or 424 & 8 & 7 \\
244 & 8 & 4 \\
422 & 5 & 5 \\
242 or 224 & 5 & 4 \\
222 & 4 & 2 \\
440,404 or 044 & 2 & 1 \\
220,202 or 022 & 1 & \\
\hline
\end{tabular}

is displayed as a function of the maximum non-zero radiation parameters. In the last column is given the number of independent linear combinations of $I_{k K M}$ terms that are measurable from the four geometries and the two angular distributions taken together. As one sees, the information from the geometries is not symmetric with respect to the three radiations. Measurements in another geometry, $\theta_{1}, \theta_{2}$ variable, $\theta_{1}+\theta_{2}$ $=\frac{1}{2} \pi, \phi_{12}=\pi$, would give additional information, with maintenance of front-back symmetry. A third detector would be needed, however, to act as radiation monitor. One more geometry, $\theta_{1}=\frac{1}{2} \pi, \theta_{2}=\frac{1}{2} \pi, \phi_{12}=$ variable, only gives additional information if terms with $K=4$ and $M=4$ occur.

It is common practice to gather so much information on a case that the parameters, describing the angular correlation, are overdetermined. In many cases, such an overdetermination leads, though not necessarily, to discrimination between different possible spin combinations. One readily sees from table 1 that angular correlations described by four variables (i.e. radiation mixture amplitudes and population para- 
meters) tend to be overdetermined only if radiation parameters of sufficiently high orders play a significant role in the angular correlation. Only then a sufficient number of independent combinations of $I_{k K M}$ can be measured. However, the geometries employed still may not give the maximum amount of information (cf., the number of relevant $I_{k K M}$ ), but do overdetermine the four variables. The $I_{k K M}$, which are zero within the rather small accuracy common in triple correlation experiments, seldom aid the discrimination between different spin combinations. In this respect multiple cascades offer more possibilities as one can often measure triple correlations between the proton direction and any two gamma rays of such a cascade, and thus obtain overdetermination of continuous parameters even in cases where only radiation parameters of low order play a significant role.

\subsection{THEORETICAL FORMULATION}

The theory of $(p, \gamma \gamma)$ angular correlations is well known. In this paper the formulation and phase definitions given by Devons and Goldfarb ${ }^{12}$ ) are used. Applications to specific problems have been evaluated, using the same formulation by Litherland and Ferguson ${ }^{8}$ ) and by Ferguson ${ }^{13}$ ). Some of their results will be quoted in the following as far as they are required for the methods of analysis used in this paper.

In addition, the existing formalism will be extended with respect to the following points: (i) a reformulation is introduced in the description of triple correlations, which avoids $9-j$ symbols; (ii) it will be shown that triple correlations with one intermediate radiation unobserved can be described in a number of cases with the existent tabulations; (iii) a simple description of angular distributions of coincident sum pulses will be presented.

The triple angular correlation between two gamma rays and the incoming protons can be generally described by a development into a series of $X_{K M}^{N}$ functions:

$$
W\left(\theta_{1}, \theta_{2}, \phi_{12}\right)=\left[e_{1} e_{2} /(4 \pi)^{2}\right] \sum_{K M N} a_{K M}^{N} Q_{K} Q_{M} X_{K M}^{N}\left(\theta_{1}, \theta_{2}, \phi_{12}\right) .
$$

The $X_{K M}^{N}$ functions are defined as

$$
\begin{aligned}
X_{K M}^{N}\left(\theta_{1}, \theta_{2}, \phi_{12}\right)=\left\{\frac{(2 K+1)(K-N) !(2 M+1)(M-N) !}{(K+N) !(M+N) !}\right\}^{\frac{1}{2}} & \\
& \quad \times P_{K}^{N}\left(\cos \theta_{1}\right) P_{M}^{N}\left(\cos \theta_{2}\right) \cos \left(N \phi_{12}\right) .
\end{aligned}
$$

with $K, M$ and $N \geqq 0$. In the present investigation the reactions proceed through levels with sharp spins and parities, so that $K$ and $M$ are even. The definition of the $X_{K M}^{N}$ functions implies that $X_{O 0}^{0}\left(\theta_{1}, \theta_{2}, \phi_{12}\right)=1$. The angles $\theta_{1}, \theta_{2}$ and $\phi_{12}$ are defined in fig. 1; they denote the angular positions of the detector axes. The angles $\theta_{1}$ and $\theta_{2}$ represent angles with respect to a reference axis, which is usually taken to be the proton-beam direction, whereas $\phi_{12}$ denotes the relative azimuthal angle of the two detectors. The $P_{K}^{N}(\cos \theta)$ are associated Legendre polynomials, as defined in ref. ${ }^{17}$ ). The factors $Q_{K}$ and $Q_{M}$ represent attenuation factors, accounting for the 
finite solid angle of the cylindrical detectors used. The symbols $e_{1}$ and $e_{2}$ denote the effective solid angles in sr of the detectors for $\gamma_{1}$ and $\gamma_{2}$. The coefficients $a_{K M}^{N}$ contain all nuclear information needed and are customarily so defined that the total emitted radiation intensity is normalized to unity.

If the measurements are performed in a geometry $G$, only one of the three angles is varied at a time. In this investigation either $\theta_{1}$ or $\theta_{2}$ was varied for each geometry (see subsect. 2.1). Then the $X_{K M}^{N}$ functions are in fact functions of only one variable, i.e., the angle $\theta\left(\theta_{1}\right.$ or $\theta_{2}$ depending on the geometry) with respect to the incoming proton-beam direction, so that development in Legendre polynomials $P_{r}(\cos \theta)$ is possible:

$$
X_{K M}^{N G}\left(\theta_{1}, \theta_{2}, \phi_{12}\right)=X_{K M}^{N G}(\theta)=\sum_{r} \alpha_{r K M N}^{\mathrm{G}} P_{r}(\cos \theta)
$$

In the geometries used in the present investigation, one has the relations $\theta_{1}=\frac{1}{2} \pi$ or $\theta_{2}=\frac{1}{2} \pi$, so that $N$ and $r$ take even values only. Furthermore one has the inequalities $N \leqq K$ and $N \leqq M$, while $r \leqq K$ if $\theta=\theta_{1}$ and $r \leqq M$ if $\theta=\theta_{2}$. An angular correlation measurement in a geometry $\mathrm{G}$ can thus be described by

$$
W^{\mathrm{G}}(\theta)=\sum_{r} B_{r}^{\mathrm{G}} P_{r}(\cos \theta)
$$

where the coefficients $B_{r}^{G}$ can be found from eqs. (2) and (3);

$$
B_{r}^{\mathrm{G}}=\left[e_{1} e_{2} /(4 \pi)^{2}\right] \sum_{K M N} a_{K M}^{N} Q_{K} Q_{M} \alpha_{r K M N}^{\mathrm{G}} .
$$

The expansion coefficients $\alpha_{r K M N}^{\mathrm{G}}$ have been tabulated by Ferguson and Rutledge ${ }^{18}$ ) for $K, M \leqq 4$ and by Heyligers ${ }^{5}$ ) for $K, M \leqq 6$, for a few standard geometries.

Angular correlation measurements are usually normalized for each geometry:

$$
W^{\mathrm{G}}(\theta)=1+A_{2}^{\mathrm{G}} P_{2}(\cos \theta)+A_{4}^{\mathrm{G}} P_{4}(\cos \theta)+\ldots
$$

where $A_{r}^{\mathrm{G}}=B_{r}^{\mathrm{G}} / B_{0}^{\mathrm{G}}$. In the analysis these experimental values of $A_{r}^{\mathrm{G}}$ are to be compared with the expressions resulting from eq. (4).

The angular distributions (double correlations) are described by

$$
\begin{aligned}
& W_{1}\left(\theta_{1}\right)=\left(e_{1} / 4 \pi\right) \sum_{r} a_{r 0}^{0} Q_{r}(2 r+1)^{\frac{1}{2}} P_{r}\left(\cos \theta_{1}\right), \\
& W_{2}^{\prime}\left(\theta_{2}\right)=\left(e_{2} / 4 \pi\right) \sum_{r} a_{0 r}^{0} Q_{r}(2 r+1)^{\frac{1}{2}} P_{r}\left(\cos \theta_{2}\right) .
\end{aligned}
$$

The symbols $a_{K M}^{N}$, containing the information on the nuclear process, can be evaluated as a function of reduced transition matrix elements and of alignment parameters of the highest excited nuclear level under consideration (usually the resonance level).

The formulation will be presented for the most often occurring triple angular correlations in the occupation number and also in the statistical tensor representation for the description of the alignment of the resonance level (see refs. ${ }^{8,9}$ )). For the decay as indicated in fig. 2 , the angular correlation between $\gamma_{1}$ and $\gamma_{2}$ is described by 
the coefficients

$$
\begin{aligned}
& a_{K M}^{N}\left(\gamma_{1} \gamma_{2}\right)=\sum_{m} p(m) \sum_{L_{1} \leqq L_{1}^{\prime} 1} \sum_{L_{2} \leqq L^{\prime} 2} C_{K M}^{N}\left(J_{1} J_{2} J_{3} L_{1} L_{1}^{\prime} L_{2} L_{2}^{\prime} m\right) \\
& \times \frac{x_{1}^{L_{1}+L_{1}^{\prime}-2 L_{1 \mathrm{~min}}} x_{2}^{L_{2}+L_{2}^{\prime}-2 L_{2 \mathrm{~min}}}}{\left(1+x_{1}^{2}\right)\left(1+x_{2}^{2}\right)} .
\end{aligned}
$$

In the present investigation there was no indication that more than the lowest two multipoles were needed for the description of a gamma transition. The symbol $x$

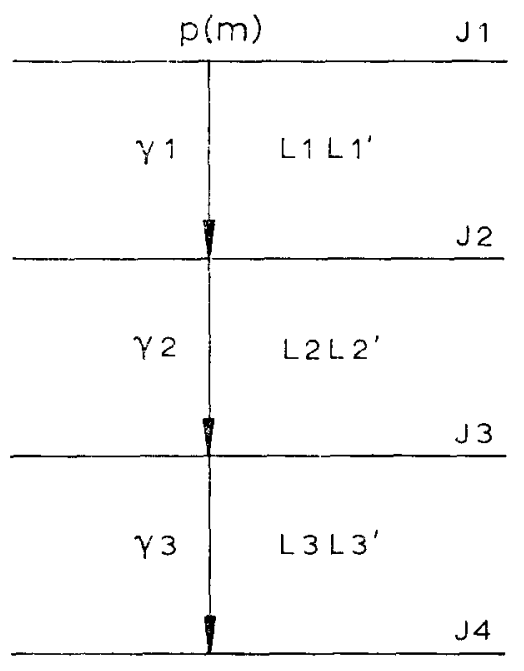

Fig. 2. Definition of spins and multipolarities in a triple cascade.

gives the ratio of the reduced transition matrix elements of the two lowest possible multipoles. In the notation of ref. ${ }^{12}$ )

$$
x_{1}=\frac{\left\langle J_{1}\left\|L_{1 \min }+1\right\| J_{2}\right\rangle}{\left\langle J_{1}\left\|L_{1 \min }\right\| J_{2}\right\rangle}, \quad x_{2}=\frac{\left\langle J_{2} \| L_{2 \min }+1|| J_{3}\right\rangle}{\left\langle J_{2}\left\|L_{2 \min }\right\| J_{3}\right\rangle} .
$$

As the reaction is symmetric with respect to reflection of the proton-beam direction, it is sufficient for a description of the alignment of the resonance level to introduce occupation numbers $p(m)$ for positive $m$-values only: $p(m)=\bar{p}\left(m_{1}\right)+\bar{p}\left(-m_{1}\right)$, where $\bar{p}\left(m_{1}\right)$ denotes the occupation probability of the magnetic sublevel $m_{1}$, and where $m=\left|m_{1}\right|$ and $-J_{1} \leqq m_{1} \leqq+J_{1}$. The dependence of $p(m)$ on the channel-spin mixture and the relevant orbital momenta has been treated concisely by Gove ${ }^{19}$ ) and also by Smith ${ }^{9}$ ).

The $C_{K M}^{N}$ coefficients have been defined by Smith ${ }^{9}$ ):

$$
\begin{aligned}
& C_{K M}^{N}\left(J_{1} J_{2} J_{3} L_{1} L_{1}^{\prime} L_{2} L_{2}^{\prime} m\right)=\left(2-\delta_{L_{1} L_{1}^{\prime}}\right)\left(2-\delta_{L_{2} L_{2}^{\prime}}\right) \\
& \quad \times(-1)^{L_{1}^{\prime}+L^{\prime}{ }_{2}-L_{2}+J_{3}-J_{2}+M+N}\left(2 J_{2}+1\right)\left\{\left(2 L_{1}+1\right)\left(2 L_{1}^{\prime}+1\right)\left(2 L_{2}+1\right)\left(2 L_{2}^{\prime}+1\right)\right\}^{\frac{1}{2}}
\end{aligned}
$$




$$
\begin{aligned}
& \times W\left(J_{2} J_{2} L_{2} L_{2}^{\prime} ; M J_{3}\right)\left\langle L_{1} 1 L_{1}^{\prime}-1 \mid K 0\right\rangle\left\langle L_{2} 1 L_{2}^{\prime}-1 \mid M 0\right\rangle \\
& \times\left[\sum_{k}\left(2-\delta_{N 0}\right)\left(2 J_{1}+1\right)(-1)^{J_{1}-m}\left\langle J_{1} m J_{1}-m \mid k 0\right\rangle\langle K N M-N \mid k 0\rangle\right. \\
& \left.\times\left(\begin{array}{lll}
J_{2} & J_{2} & M \\
L_{1} & L_{1}^{\prime} & K \\
J_{1} & J_{1} & k
\end{array}\right)\right]
\end{aligned}
$$

where the standard notation for Clebsch-Gordan coefficients, Racah-coefficients and 9-j symbols has been used. In the paper by Smith ${ }^{9}$ ) the $C_{K M}^{N}$ coefficients are tabulated for a large number of spin combinations.

In the computations for the present investigation a slightly different formula was used. In eq. (6) the summation over $k$ results from a description of the resonance level in terms of tensor parameters, giving rise to a $9-j$ symbol. This tensor expansion can be avoided, so that the part of eq. (6) in square brackets can be replaced by

$$
\begin{aligned}
& {\left[\sum_{k}\left(2-\delta_{N 0}\right)\left(2 J_{1}+1\right)(-1)^{J_{1}-m}\left\langle J_{1} m j_{1}-m \mid k 0\right\rangle\langle K N M-N \mid k 0\rangle\left(\begin{array}{ccc}
J_{2} & J_{2} & M \\
L_{1} & L_{1}^{\prime} & K \\
J_{1} & J_{1} & k
\end{array}\right)\right]} \\
& =\left[\sum_{N^{\prime}} \sum_{-J_{2} \leqq m_{2}, m^{\prime} \leqq}(-1)^{J_{2}-L_{1}^{\prime}-m}\{(2 K+1)(2 M+1)\}^{-\frac{1}{2}}\right. \\
& \quad \times\left\{\delta_{N N^{\prime}}+\left(1-\delta_{N 0}\right) \delta_{N-N^{\prime}}\right\}\left\langle J_{2} m_{2} L_{1} m-m_{2} \mid J_{1} m\right\rangle\left\langle J_{2} m_{2}^{\prime} L_{1}^{\prime} m-m_{2}^{\prime} \mid J_{1} m\right\rangle \\
& \left.\quad \times\left\langle J_{2} m_{2} J_{2}-m_{2}^{\prime} \mid M N^{\prime}\right\rangle\left\langle L_{1} m-m_{2} L_{1}^{\prime} m_{2}^{\prime}-m \mid K-N^{\prime}\right\rangle\right] .
\end{aligned}
$$

The summation over $N^{\prime}$ leads to only two terms $(N \neq 0)$ or one $(N=0)$. Each of these terms consists of a single summation since the Clebsch-Gordan coefficients in the right-hand side contains a delta function $\delta_{m_{2}-m_{2}}, N^{\prime}$. Now the right-hand side of eq. (7) allows for faster computer computation, as it contains no coefficients depending on more than four different symbols.

The angular correlation between $\gamma_{1}$ and $\gamma_{3}$ (see fig. 2) can be described in terms of the coefficients

$$
\begin{aligned}
a_{K M}^{N}\left(\gamma_{1} \gamma_{3}\right)=\sum_{m} p(m) \sum_{L_{1} \leqq L_{1}^{\prime}} \sum_{L_{2}} \sum_{L_{3} \leqq L_{3}^{\prime}} G_{K M}^{N}\left(J_{1} J_{2} J_{3} J_{4} L_{1} L_{1}^{\prime} L_{2} L_{3} L_{3}^{\prime} m\right) \\
\times \frac{x_{1}^{L_{1}+L_{1}^{\prime}-2 L_{1 \min }} x_{2}^{2\left(L_{2}-L_{2 \min }\right)} x_{3}^{L_{3}+L^{\prime}{ }_{3}-2 L_{3 \mathrm{~min}}}}{\left(1+x_{1}^{2}\right)\left(1+x_{2}^{2}\right)\left(1+x_{3}^{2}\right)},
\end{aligned}
$$

where

$$
\begin{aligned}
G_{K M}^{N} & \left(J_{1} J_{2} J_{3} J_{4} L_{1} L_{1}^{\prime} L_{2} L_{3} L_{3}^{\prime} m\right)=\left(2-\delta_{L_{1} L_{1}^{\prime}}\right)\left(2-\delta_{L_{3} L_{3}^{\prime}}\right) \\
& \times(-1)^{L_{1}^{\prime}+L_{3}^{\prime}-L_{3}+L_{2}-J_{2}-2 J_{3}+J_{4}+2 M+N}\left(2 J_{2}+1\right)\left(2 J_{3}+1\right) \\
& \times\left\{\left(2 L_{1}+1\right)\left(2 L_{1}^{\prime}+1\right)\left(2 L_{3}+1\right)\left(2 L_{3}^{\prime}+1\right)\right\}^{\frac{1}{2}} W\left(J_{2} J_{2} J_{3} J_{3} ; M L_{2}\right) \\
& \times W\left(J_{3} J_{3} L_{3} L_{3}^{\prime} ; M J_{4}\right)\left\langle L_{1} 1 L_{1}^{\prime}-1 \mid K 0\right\rangle\left\langle L_{3} 1 L_{3}^{\prime}-1 \mid M 0\right\rangle
\end{aligned}
$$




$$
\begin{aligned}
& \times\left[\sum_{k}\left(2-\delta_{N 0}\right)\left(2 J_{1}+1\right)(-1)^{J_{1}-m}\left\langle J_{1} m J_{1}-m \mid k 0\right\rangle\langle K N M-N \mid k 0\rangle\right. \\
& \left.\times\left(\begin{array}{lll}
J_{2} & J_{2} & M \\
L_{1} & L_{1}^{\prime} & K \\
J_{1} & J_{1} & k
\end{array}\right)\right] .
\end{aligned}
$$

Again the part in square brackets can be replaced as given in eq. (7). In cases where $J_{4}=0$, eq. (9) can be simplified to

$$
\begin{aligned}
& G_{K M}^{N}\left(J_{1} J_{2} J_{3} 0 L_{1} L_{1}^{\prime} L_{2} L_{3} L_{3} m\right)=\left(2-\delta_{L_{1} L_{1}}\right)(-1)^{L_{1}+L_{2}-J_{2}+M+N} \\
& \quad \times\left(2 J_{2}+1\right)\left(2 J_{3}+1\right)\left\{\left(2 L_{1}+1\right)\left(2 L_{1}^{\prime}+1\right)\right\}^{\frac{1}{2}} W\left(J_{2} J_{2} J_{3} J_{3} ; M L_{2}\right) \\
& \quad \times\left\langle L_{1} 1 L_{1}^{\prime}-1 \mid K 0\right\rangle\left\langle J_{3} 1 J_{3}-1 \mid M 0\right\rangle\left[\sum_{k}\left(2-\delta_{N 0}\right)\left(2 J_{1}+1\right)(-1)^{J_{1}-m}\right. \\
& \left.\quad \times\left\langle J_{1} m J_{1}-m \mid k 0\right\rangle\langle K N M-N \mid k 0\rangle\left(\begin{array}{ccc}
J_{2} & J_{2} & M \\
L_{1} & L_{1}^{\prime} & K \\
J_{1} & J_{1} & k
\end{array}\right)\right] .
\end{aligned}
$$

One readily observes that

$$
G_{K M}^{N}\left(J_{1} J_{2} J_{3} O L_{1} L_{1}^{\prime} L_{2} L_{3} L_{3} m\right)=C_{K M}^{N}\left(J_{1} J_{2} L_{2} L_{1} L_{1}^{\prime} J_{3} J_{3} m\right) .
$$

Consequently, in a number of cases the existing tables of $C_{K M}^{N}$ coefficients can be used to obtain the $G_{K M}^{N}$ coefficients numerically.

The angular correlation between $\gamma_{2}$ and $\gamma_{3}$ (see fig. 2) can be described in terms of the coefficients

$$
\begin{aligned}
a_{K M}^{N}\left(\gamma_{2} \gamma_{3}\right)=\sum_{m} p(m) \sum_{L_{1}} \sum_{L_{2} \leqq L^{\prime} 2} & \sum_{L_{3} \leqq L^{\prime}{ }_{3}} H_{K M}^{N}\left(J_{1} J_{2} J_{3} J_{4} L_{1} L_{2} L_{2}^{\prime} L_{3} L_{3}^{\prime} m\right) \\
& \times \frac{x_{1}^{2\left(L_{1}-L_{1} \mathrm{~min}\right)} x_{2}^{L^{\prime}{ }_{2}+L_{2}-2 L_{2} \mathrm{~min}} x_{3}^{L_{3}+L_{3}-2 L_{3 \mathrm{~min}}}}{\left(1+x_{1}^{2}\right)\left(1+x_{2}^{2}\right)\left(1+x_{3}^{2}\right)},
\end{aligned}
$$

where

$$
\begin{aligned}
& H_{K M}^{N}\left(J_{1} J_{2} J_{3} J_{4} L_{1} L_{2} L_{2}^{\prime} L_{3} L_{3}^{\prime} m\right)=\sum_{0 \leqq m_{2} \leqq J_{2}}\left\{\left\langle J_{2} m_{2} L_{1} m-m_{2} \mid J_{1} m\right\rangle^{2}\right. \\
& \left.\quad+\left(1-\delta_{m_{2} 0}\right)\left\langle J_{2}-m_{2} L_{1} m+m_{2} \mid J_{1} m\right\rangle^{2}\right\} C_{K M}^{N}\left(J_{2} J_{3} J_{4} L_{2} L_{2}^{\prime} L_{3} L_{3}^{\prime} m_{2}\right) .
\end{aligned}
$$

Analogous formulations for the angular correlations above can be obtained if the alignment of the resonance state is described by Fano's statistical tensors ${ }^{13}$ ), defined by

$$
\rho_{k 0}\left(J_{1} J_{1}\right)=\sum_{m}(-1)^{J_{1}-m}\left\langle J_{1} m J_{1}-m \mid k 0\right\rangle p(m) .
$$

It is sufficient to substitute this expression in eqs. (5) and (8). The expression for the 
angular correlation between $\gamma_{2}$ and $\gamma_{3}$ is most easily obtained by combining expressions (15), (16) and (22).

The inverse relation is

$$
p(m)=\sum_{k}(-1)^{J_{1}-m}\left\langle J_{1} m J_{1}-m \mid k 0\right\rangle\left(2-\delta_{m 0}\right) \rho_{k 0}\left(J_{1} J_{1}\right) .
$$

(The factor $\left(2-\delta_{m 0}\right)$ in eq. (14) arises from the definition of $p(m)$ for positive $m$ only.) The explicit expression of the angular correlation between $\gamma_{1}$ and $\gamma_{2}$ in the tensor formalism becomes

$$
\begin{aligned}
& W\left(\theta_{1}, \theta_{2}, \phi_{12}\right)=\left[e_{1} e_{2} /(4 \pi)^{2}\right] \sum_{k}\left[\rho_{k 0}\left(J_{1} J_{1}\right) / \rho_{00}\left(J_{1} J_{1}\right)\right] \\
& \times \sum_{L_{1} \leqq L^{\prime}{ }_{1}} \sum_{L_{2} \leqq L^{\prime} 2} \sum_{K M N} A_{K M}^{N}\left(J_{1} J_{2} J_{3} L_{1} L_{1}^{\prime} L_{2} L_{2}^{\prime} k\right) \\
& \times \frac{x_{1}^{L_{1}+L^{\prime} 1-2 L_{1 \mathrm{~min}}} x_{2}^{L_{2}+L_{2}-2 L_{2 \mathrm{~m} i \mathrm{n}}}}{\left(1+x_{1}^{2}\right)\left(1+x_{2}^{2}\right)}-X_{K M}^{N}\left(\theta_{1}, \theta_{2}, \phi_{12}\right) Q_{K} Q_{M},
\end{aligned}
$$

where

$$
\begin{aligned}
A_{K M}^{N}( & \left.J_{1} J_{2} J_{3} L_{1} L_{1}^{\prime} L_{2} L_{2}^{\prime} k\right)=\left(2-\delta_{L_{1} L_{1}^{\prime}}\right)\left(2-\delta_{L_{2} L_{2} 2}\right)\left(2-\delta_{N 0}\right) \\
& \times(-1)^{L_{1}^{\prime}+L_{2}^{\prime}-L_{2}+J_{3}-J_{2}+M+N}\left(2 J_{2}+1\right) \\
& \times\left\{\left(2 J_{1}+1\right)\left(2 L_{1}+1\right)\left(2 L_{1}^{\prime}+1\right)\left(2 L_{2}+1\right)\left(2 L_{2}^{\prime}+1\right)\right\}^{\frac{1}{2}}\langle K N M-N \mid k 0\rangle \\
& \times\left(\begin{array}{lll}
J_{2} & J_{2} & M \\
L_{1} & L_{1}^{\prime} & K \\
J_{1} & J_{1} & k
\end{array}\right) W\left(J_{2} J_{2} L_{2} L_{2}^{\prime} ; M J_{3}\right)\left\langle L_{1} 1 L_{1}^{\prime}-1 \mid K 0\right\rangle\left\langle L_{2} 1 L_{2}^{\prime}-1 \mid M 0\right\rangle .
\end{aligned}
$$

With this definition the total intensity of the emitted radiation is normalized to unity, as $A_{00}^{0}\left(J_{1} J_{2} J_{3} L_{1} L_{1} L_{2} L_{2} 0\right)=1$, which is also the case for the tabulation by Kaye, Read and Willmott ${ }^{20}$ ) for the $A_{K M}^{N}$ coefficients ${ }^{\dagger}$.

For the angular distribution of $\gamma_{1}$, formula (15) simplifies to

$$
W\left(\theta_{1}\right)=\sum_{k}\left[\rho_{k 0}\left(J_{1} J_{1}\right) / \rho_{00}\left(J_{1} J_{1}\right)\right] a_{k}\left(\gamma_{1}\right) P_{k}\left(\cos \theta_{1}\right)
$$

where

$$
a_{k}\left(\gamma_{1}\right)=\left(e_{1} / 4 \pi\right) \sum_{L_{1} \leqq L_{1}^{\prime}} A_{k 0}^{0}\left(J_{1} J_{2} J_{3} L_{1} L_{1}^{\prime} L_{2} L_{2} k\right) Q_{k}(2 k+1)^{\frac{1}{2}} \frac{x_{1}^{L_{1}+L^{\prime}{ }_{1}-2 L_{1 \mathrm{~min}}}}{\left(1+x_{1}^{2}\right)},
$$

whereas the angular distribution of $\gamma_{2}$ is described by

$$
W\left(\theta_{2}\right)=\sum_{k}\left[\rho_{k 0}\left(J_{1} J_{1}\right) i \rho_{00}\left(J_{1} J_{1}\right)\right] b_{k}\left(\gamma_{2}\right) P_{k}\left(\cos \theta_{2}\right)
$$

$\uparrow$ Due to a typographical error the expression for the angular correlation given by Kaye et al. ${ }^{20}$ ) does not contain the factor $\rho_{00}\left(J_{1} J_{1}\right)$ in the denominator. 
where

$$
\begin{aligned}
b_{k}\left(\gamma_{2}\right)=\left(e_{2} / 4 \pi\right) \sum_{L_{1}} \sum_{L_{2} \leqq L_{2}^{\prime}} A_{0 k}^{0}\left(J_{1} J_{2} J_{3} L_{1} L_{1} L_{2} L_{2}^{\prime} k\right) Q_{k}(2 k+1)^{\frac{1}{2}} & \\
& \times \frac{x_{1}^{2\left(L_{1}-L_{1 \mathrm{~min}}\right)} x_{2}^{L_{2}+L_{2}^{\prime}-2 L_{2 \mathrm{~min}}}}{\left(1+x_{1}^{2}\right)\left(1+x_{2}^{2}\right)} .
\end{aligned}
$$

The tensor formalism is especially suitable for a description of the angular correlations in steps, corresponding to the successive emission of radiation. After the emission and detection of the first gamma ray in the decay process (see fig. 2), the statistical tensors of the level with spin $J_{2}$ are described by

$$
\begin{aligned}
& \rho_{M N}\left(J_{2} J_{2}\right)=e_{1}(4 \pi)^{-\frac{1}{2}} \sum_{k K} \rho_{k 0}\left(J_{1} J_{1}\right) \sum_{L_{1} \leqq L_{1}^{\prime}}\left(2-\delta_{L^{\prime}{ }_{1} L_{1}}\right)(-1)^{L_{1}-1}\left(2 J_{1}+1\right) \\
& \quad \times\left\{\left(2 L_{1}+1\right)\left(2 L_{1}^{\prime}+1\right)(2 M+1)\right\}^{\frac{1}{2}}\langle K N M-N \mid k 0\rangle\left(\begin{array}{lll}
J_{2} & J_{2} & M \\
L_{1} & L_{1}^{\prime} & K \\
J_{1} & J_{1} & k
\end{array}\right) \\
& \quad \times\left\langle L_{1} 1 L_{1}^{\prime}-1 \mid K 0\right\rangle \frac{x_{1}^{L_{1}+L^{\prime}{ }_{1}-2 L_{1} \min }}{\left(1+x_{1}^{2}\right)} Q_{K} Y_{K}^{-N}\left(\theta_{1}, \phi_{1}\right) .
\end{aligned}
$$

The angular dependence of the intensity of the second gamma ray from the decay of the level with spin $J_{2}$ is given by

$$
\begin{aligned}
& W\left(\theta_{2}, \phi_{2}\right)=e_{2}(4 \pi)^{-\frac{1}{2}} \sum_{M} \sum_{-M \leqq N \leqq+M} \rho_{M N}\left(J_{2} J_{2}\right) \sum_{L_{2} \leqq L_{2}^{\prime}}\left(2-\delta_{L^{\prime}{ }_{2} L_{2}}\right) \\
& \quad \times(-1)^{L_{2}{ }_{2}-L_{2}+J_{3}-J_{2}+M-1}\left(2 J_{2}+1\right)\left\{\left(2 L_{2}+1\right)\left(2 L_{2}^{\prime}+1\right) /(2 M+1)\right\}^{\frac{1}{2}} \\
& \quad \times W\left(J_{2} J_{2} L_{2} L_{2}^{\prime} ; M J_{3}\right)\left\langle L_{2} 1 L_{2}^{\prime}-1 \mid M 0\right\rangle \frac{x_{2}^{L_{2}+L_{2}^{\prime}-2 L_{2 \mathrm{~min}}}}{\left(1+x_{2}^{2}\right)} Q_{M} Y_{M}^{N}\left(\theta_{2}, \phi_{2}\right)
\end{aligned}
$$

The explicit dependence on the angles $\theta_{2}$ and $\phi_{12}$ is obtained if one substitutes into eq. (20) the expression

$$
\begin{aligned}
Y_{M}^{N}\left(\theta_{2}, \phi_{2}\right)=(4 \pi)^{-\frac{1}{2}}(-1)^{\frac{1}{2}(N+|N|)}\{(2 M+1)(M-|N|) !(M & +|N|) !\}^{\frac{1}{2}} \\
& \times P_{M}^{|N|}\left(\cos \theta_{2}\right) \exp \left[i N \phi_{2}\right],
\end{aligned}
$$

(see ref. $\left.{ }^{13}\right)$ ), and a similar expression for $Y_{K}^{-N}\left(\theta_{1}, \phi_{1}\right)$ into eq. (19). Thus we find, writing $\rho_{M N}\left(J_{1} J_{2}\right)=\left|\rho_{M N}\left(J_{1} J_{2}\right)\right| \exp \left[-i N \phi_{1}\right]$, for the angular dependence

$$
\begin{aligned}
& W\left(\theta_{2}, \phi_{12}\right)=\left(e_{2} / 4 \pi\right) \sum_{M} \sum_{0 \leqq N \leqq M}\left|\rho_{M N}\left(J_{2} J_{2}\right)\right| \sum_{L_{2} \leqq L_{2}^{\prime}}\left(2-\delta_{L_{2}^{\prime} L_{2}}\right)\left(2-\delta_{N 0}\right) \\
& \quad \times(-1)^{L_{2}-L_{2}+J_{3}-J_{2}+M+N-1}\left(2 J_{2}+1\right)\left\{\left(2 L_{2}+1\right)\left(2 L_{2}^{\prime}+1\right)\right\}^{\frac{1}{2}} \\
& \quad \times W\left(J_{2} J_{2} L_{2} L_{2}^{\prime} ; M J_{3}\right)\left\langle L_{2} 1 L_{2}^{\prime}-1 \mid M 0\right\rangle Q_{M}\{(M-N) ! /(M+N) !\}^{\frac{1}{2}} \\
& \quad \times P_{M}^{N}\left(\cos \theta_{2}\right) \cos \left(N \phi_{12}\right),
\end{aligned}
$$

where

$$
\phi_{12}=\phi_{1}-\phi_{2}
$$


If the second emitted radiation is not observed the statistical tensor component $\rho_{M N}$ of the level with spin $J_{3}$ becomes

$$
\begin{aligned}
\rho_{M N}\left(J_{3} J_{3}\right)=\rho_{M N}\left(J_{2} J_{2}\right)\left(2 J_{2}+1\right) \sum_{L_{2}}( & -1)^{L_{2}+M-J_{2}-J_{3}} \\
& \times\left|\left\langle J_{3}|| L_{2} \| J_{2}\right\rangle\right|^{2} W\left(J_{2} J_{2} J_{3} J_{3} ; M L_{2}\right) .
\end{aligned}
$$

The statistical tensor components $\rho_{M N}\left(J_{3} J_{3}\right)$ are proportional to $\rho_{M N}\left(J_{2} J_{2}\right)$. The generalization to multistep decays is evident. This formulation can also be used if types of radiation other than gamma rays are emitted. In the case of non-observed proton emission, $L_{2}$ should be replaced by $j_{\mathrm{p}}$.

In the case of coincident summing of the pulses from two gamma rays in a detector, the angular distribution to be measured can be considered as a special geometry of a triple correlation. Substitution of $\theta_{1}=\theta_{2}=\theta$ and $\phi_{12}=0$ in eq. (15) then yields for the angular distribution

$$
W(\theta)_{\text {sum }}=\sum_{k}\left[\rho_{k 0}\left(J_{1} J_{1}\right) / \rho_{00}\left(J_{1} J_{1}\right)\right] a_{k \text { sum }} P_{k}(\cos \theta),
$$

where

$$
\begin{aligned}
a_{k \text { sum }} & =\left[e_{1} e_{2} /(4 \pi)^{2}\right] \sum_{L_{1} \leqq L^{\prime} 1} \sum_{L_{2} \leqq L^{\prime}} \sum_{K M} A_{K M}^{0}\left(J_{1} J_{2} J_{3} L_{1} L_{1}^{\prime} L_{2} L_{2}^{\prime} k\right) \\
& \times\{(2 K+1)(2 M+1)\}^{\frac{1}{2}} Q_{K} Q_{M} \frac{x_{1}^{L_{1}+L^{\prime}{ }^{\prime}-2 L_{1 \mathrm{~min}}} x_{2}^{L_{2}+L_{2}^{\prime}-2 L_{2 \mathrm{mln}}}}{\left(1+x_{1}^{2}\right)\left(1+x_{2}^{2}\right)} .
\end{aligned}
$$

The formulae for coincident summing of $\gamma_{1}$ and $\gamma_{3}$ and of $\gamma_{2}$ and $\gamma_{3}$ are similar.

Throughout this investigation $X_{K M}^{N}$ functions are used for the description of triple correlations. Alternatively, Biedenharn's more symmetric $P_{k K M}$ functions ${ }^{16}$ ) can be used. These are related to the $X_{K M}^{N}$ functions by

$$
\begin{aligned}
P_{k K M}\left(0, \Omega_{1}, \Omega_{2}\right)=i^{k+K-M} /\left\{(2 K+1)^{\frac{1}{2}}(2 M+1)\right\} & \\
& \times \sum_{N \geqq 0}\left(2-\delta_{N 0}\right)\langle k 0 K N| M N>X_{K M}^{N}\left(\theta_{1}, \theta_{2}, \phi_{12}\right),
\end{aligned}
$$

where $\Omega_{1}=\theta_{1}, \phi_{2}$ and $\Omega_{2}=\theta_{2}, \phi_{2}$ (see ref. $\left.{ }^{13}\right)$ ). The index $N$ can thus be avoided in most formulae. Tabulations using $P_{k K M}$ functions have been made by Ferguson ${ }^{13}$ ).

\subsection{METHOD OF ANALYSIS}

The analysis proceeds in two steps. In the first step, describing the $\gamma$-decay of the resonance level, the population probabilities $p(m)$ of the magnetic substates of the resonance level and the mixing ratios $x_{j}$ of the $\gamma$-transitions are treated as unknown parameters, to be determined by a least-squares analysis of the experimental data. This method is due to Litherland and Ferguson ${ }^{8}$ ). The necessary numerical coefficients have been partly tabulated by Smith ${ }^{9}$ ) and by Kaye, Read and Willmott ${ }^{20}$ ). For our analysis, however, we have computed the coefficients mostly with ALGOL programs, using the formulae mentioned in subsect. 2.2. In this way the errors made 
in copying a large number of coefficients were avoided. A useful summary of leastsquares techniques is given in ref. ${ }^{21}$ ).

The unknown spins are treated as discrete parameters. They are determined by performing the analysis for different spin values, and then taking the solution with the lowest value of $\chi^{2}$. A solution is considered unique if all other solutions give $\chi^{2}$ values above the $0.1 \%$ probability limit (see ref. ${ }^{21}$ )). The programs of analysis, described in this section, are especially suitable for a combined analysis of several steps in a multiple cascade and of several cascades per resonance.

The second step in the analysis treats the formation of the resonant state through proton capture. The observed population probabilities are interpreted as functions of the channel-spin mixing parameter $\tau$ and the orbital mixing parameters $\varepsilon_{1}$ and $\varepsilon_{2}$ in the competing $1^{+}$and $2^{+}$channels (following from the $\frac{3}{2}{ }^{+35} \mathrm{Cl}$ ground-state spin and parity.) The phase shifts ${ }^{19}$ ) between $s$ and $d, p$ and $f, d$ and $g$ waves, etc. have to be taken into account. The mixing parameter $\varepsilon_{i}$ is the amplitude ratio of the proton partial wave with high orbital momentum over that with the lowest orbital momentum in the channel $i$. The quantity $\tau$ is defined as the ratio of the total intensity in the $1^{+}$ channel over the total intensity in both channels together. In most cases the number of independent population parameters is smaller than the number of formation parameters $\left(\tau, \varepsilon_{1}, \varepsilon_{2}\right)$, so that the latter are underdetermined.

\subsubsection{Analysis of the $\gamma$-decay}

To begin with, the $A_{2}$ and $A_{4}$ coefficients for each geometry are computed with their statistical errors from the measurements at four angles per geometry. In the computation of the errors, the statistical fluctuations in the measured background and of the

TABLE 2

Spins $\left(J_{i}\right)$ and largest significant multipole orders $\left(l_{\mathrm{pmax}}, L_{1 \mathrm{max}}\right)$, for which minimum values can be deduced from the significance of the coefficients $A_{r} G$, (with $r=2,4, \ldots$ ), for the geometries $\mathrm{G}=\mathrm{I}, \mathrm{II}, \mathrm{V}, \mathrm{VI}$, Angular distribution 1 and Angular distribution 2. The minimum values are equal to $\frac{1}{2} r$

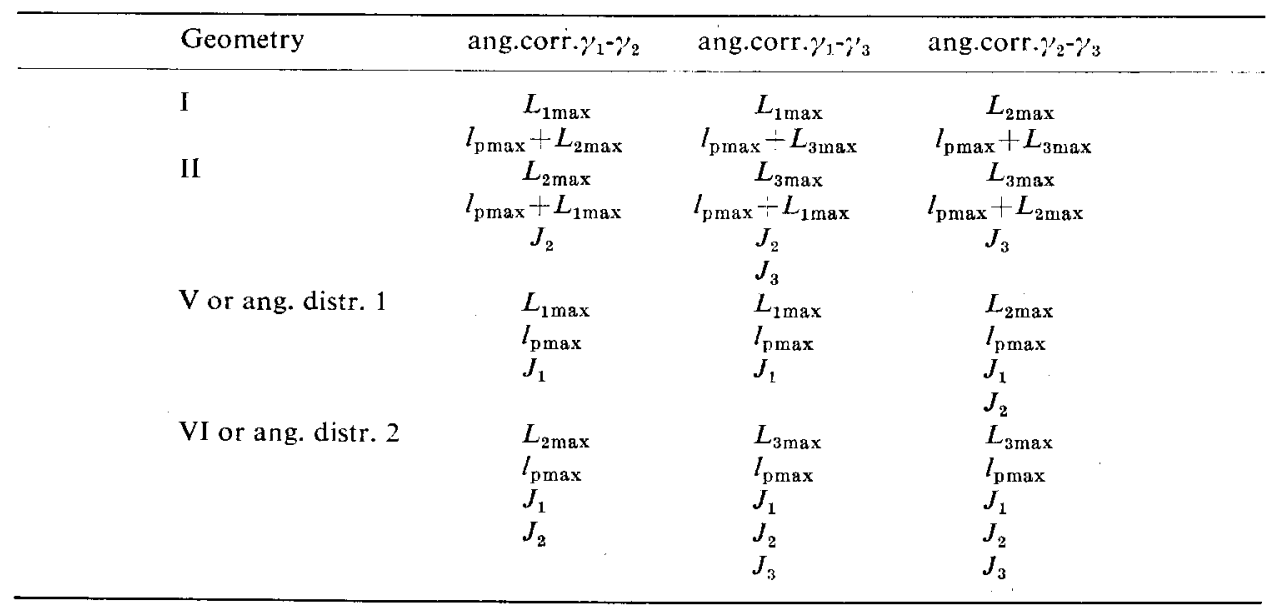


corrections are taken into account. From the values of the $A_{2}$ and $A_{4}$ coefficients some general conclusions can be drawn concerning the minimum values of spins and radiation multipole orders $L_{\max }$ which are still significant. In table 2 the conclusions are summarized for measurements in the geometries I, II, V and VI and of the angular distributions, for the angular correlations $\gamma_{1}-\gamma_{2}, \gamma_{1}-\gamma_{3}$ and $\gamma_{2}-\gamma_{3}$ in the cascade $J_{1}-J_{2}-J_{3}-J_{4}$ (see fig. 2). An $A_{2}$ or $A_{4}$ coefficient can be considered as significantly different from zero, if its absolute value is larger than three times the experimental error. This corresponds to about the $0.1 \%$ probability limit, as used in the $\chi^{2}$ method.

As a following step, one searches for the best values of the continuous parameters in a systematic trial and error analysis, using the $\chi^{2}$ method. The possible spin values are handled as discrete parameters. The occupation probabilities and radiation mixing ratios have no linear relation to the measured $A_{2}$ and $A_{4}$ coefficients, which makes the analysis complicated. In the literature, several methods have been mentioned for a least-squares analysis of a single cascade. Heyligers ${ }^{5}$ ) and also Broude and Gove ${ }^{10}$ ) have introduced non-linear, least-squares methods. These have the disadvantage that secondary minima in $\chi^{2}$ may remain unnoticed. Glaudemans and Endt ${ }^{6}$ ) have varied the radiation mixing ratios in finite steps and linearized the resulting least-squares equations by normalization of the measured geometries and angular distributions, so that the occupation probabilities $p(m)$ could be obtained as a straightforward solution, according to a method introduced by Smith ${ }^{9,22}$ ). Though this method is fast and satisfactory in the cases investigated, some drawbacks are evident; (i) the normalization of the geometries more or less arbitrarily eliminates possible secondary minima in $\chi^{2}$, and correlations between the normalized measurements are neglected; (ii) the solutions for the occupation probabilities frequently are outside the physically admissible region, and corrections for this are difficult to make; (iii) especially for the present investigation the method becomes too complicated when a least-squares solution is desired for measurements on several cascades at a resonance.

In the analysis employed in the present investigation, all parameters are varied in finite steps. For each combination of parameter values tried, a value of $\chi^{2}$ is computed according to

$$
\chi^{2}=\sum_{\mathrm{G}} \sum_{i, j} X_{i j}^{\mathrm{G}}\left(A_{i}^{\mathrm{G}}-A_{i \mathrm{tr}}^{\mathrm{G}}\right)\left(A_{j}^{\mathrm{G}}-A_{j \mathrm{tr}}^{\mathrm{G}}\right), \quad \text { with } i \text { and } j=2,4 .
$$

The $A_{i}^{\mathrm{G}}$ are the measured values of $A_{i}$ for geometry $\mathrm{G}$. The $X_{i j}^{\mathrm{G}}$ are weighting factors, equal to elements of the inverse error matrices for $A_{i}^{\mathrm{G}}$. The $A_{i \text { tr }}^{\mathrm{G}}$ are the trial values that follow from specific values of the parameters. For a good fit to the measurements, the normalized $\chi^{2}$, denoted by $\chi_{n}^{2} \equiv \chi^{2} /(N-M)$, should be of the order of unity. In this expression, $N$ is the number of $A_{2}$ and $A_{4}$ coefficients and $M$ the number of continuously variable parameters. Apparently, it is not relevant whether the equations for the $A_{2}$ and $A_{4}$ coefficients are linear in the parameters. The method, in the versions worked out up to now, with channel-spin values up to 2 , is about four times slower than Smith's method in the case of an analysis of a single cascade. 


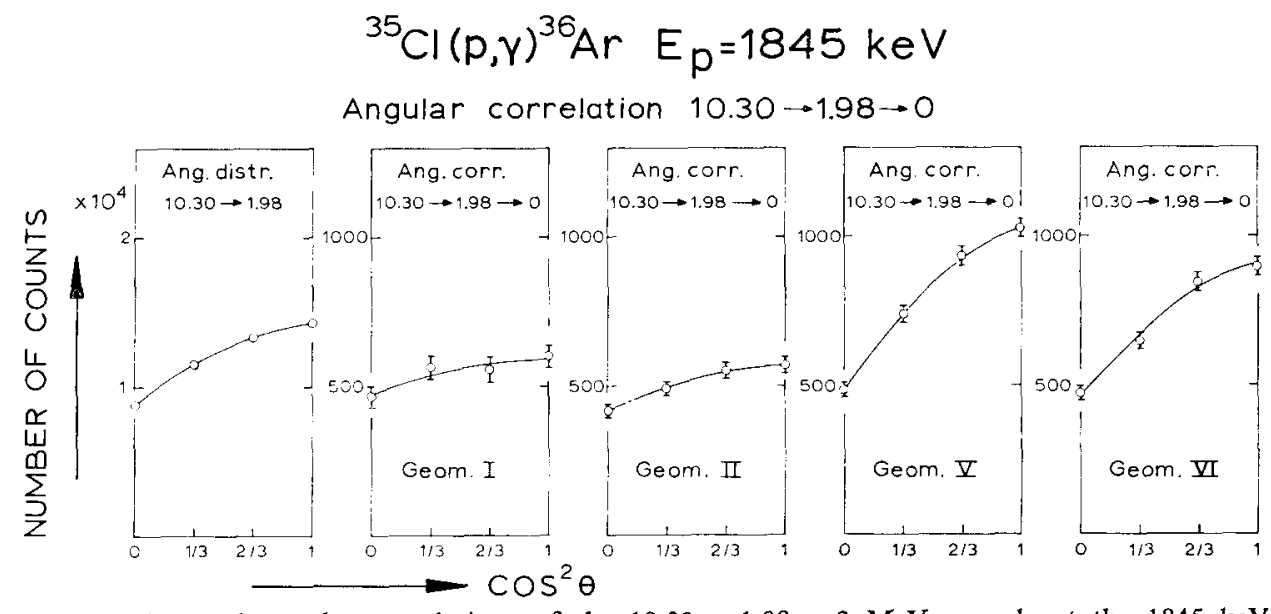

Fig. 3. Measured angular correlations of the $10.30 \rightarrow 1.98 \rightarrow 0 \mathrm{MeV}$ cascade at the $1845 \mathrm{keV}$ resonance. The solid lines give the best fits with the expansion $W(\theta)=B_{0}+B_{2} P_{2}(\cos \theta)+B_{4} P_{4}(\cos \theta)$

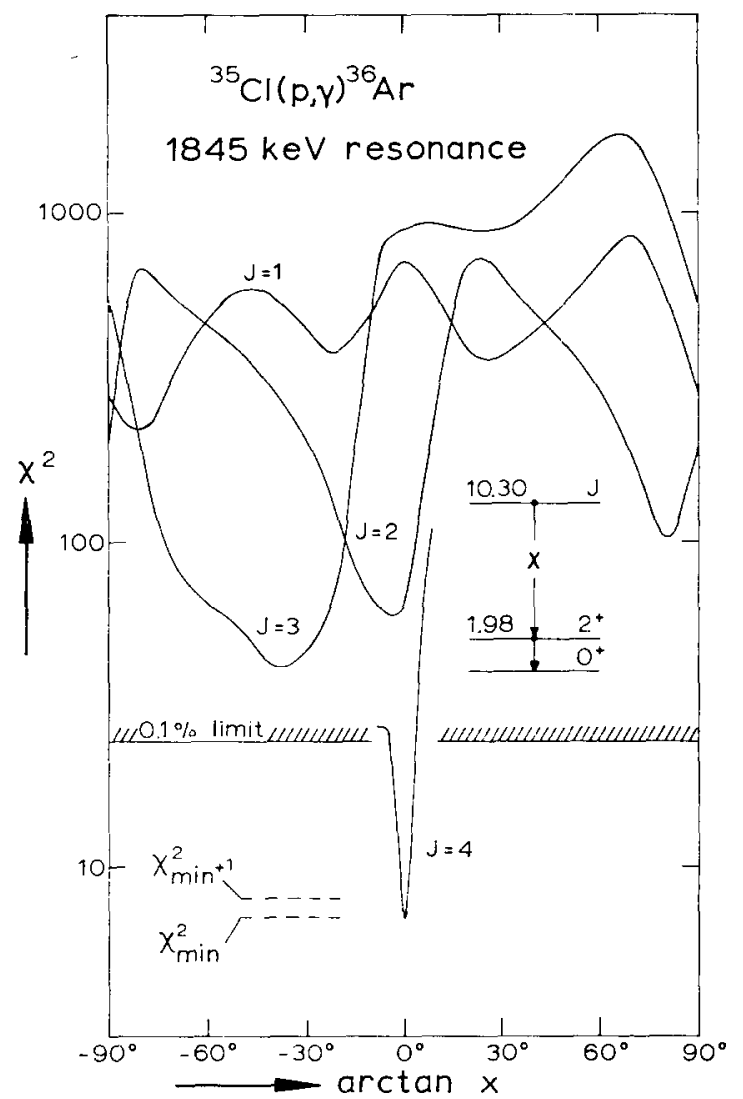

Fig. 4. Unnormalized values of $\chi^{2}$ from $\gamma$-ray double and triple correlation measurements of the $10.30 \rightarrow 1.98 \rightarrow 0 \mathrm{MeV}$ cascade at the $1845 \mathrm{keV}$ resonance, plotted against the radiation mixing ratio of the first transition with the resonance spin $J$ as a parameter. For the meaning of the $0.1 \%$ probability limit, see the text. 
The systematic search for the best values of the parameters is accomplished as follows. Dependent on the spin of the resonant state, one has occupation probabilities $p(0)+p(1)=1$ (for $J=1$ ) or $p(0)+p(1)+p(2)=1$ (for $J>1$ ), as the reaction proceeds via the channel spins $1^{+}$and $2^{+}$. In the ALGOL computer program the occupation probabilities are varied in steps of 0.05 and 0.1 , respectively. These steps were found to be small enough to determine a minimum. For each combination of the radiation-mixing ratio values $x_{j}$ one thus computes 21 and 66 values of $\chi^{2}$, respectively.

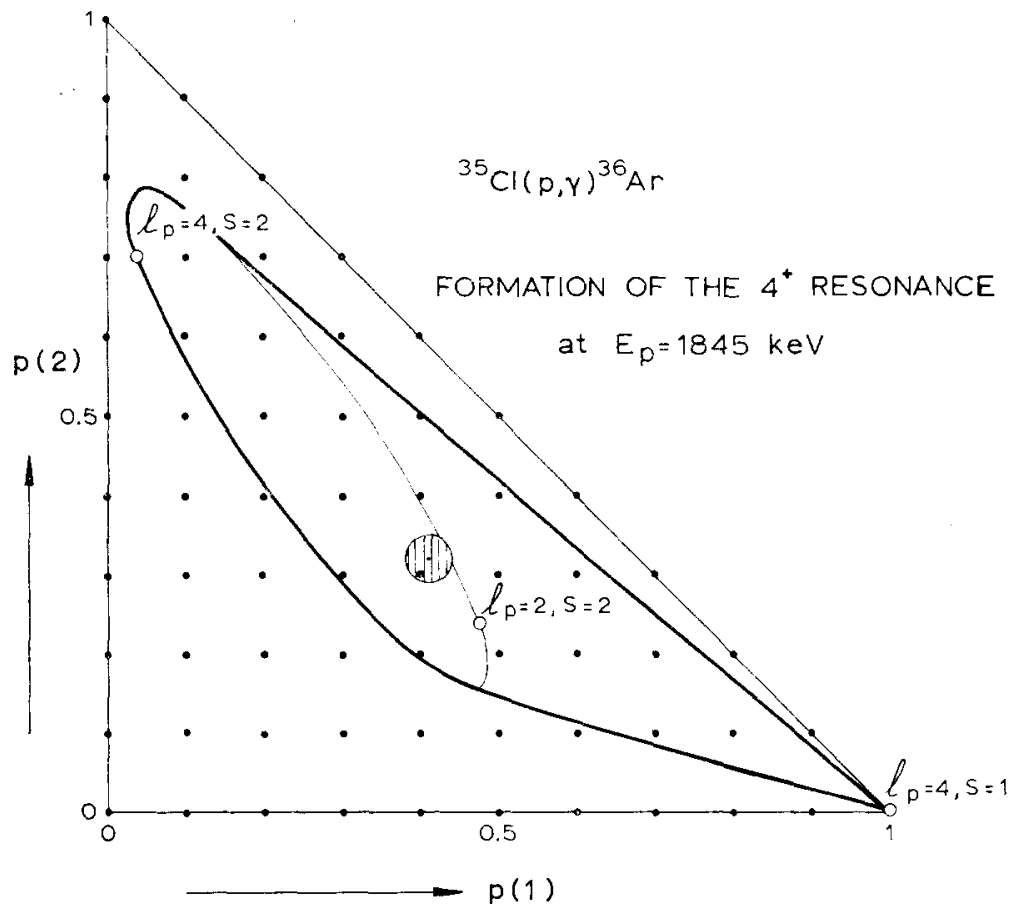

Fig. 5. Formation of the $4^{+}$resonance at $E_{\mathrm{p}}=1845 \mathrm{keV}$. The occupation probabilities $p(1)$ and $p(2)$ (with $p(0)=1-p(1)-p(2)$ ) and their errors, obtained from the least-squares fit to the angular correlation measurements, are indicated as a hatched region. The theoretically possible solutions for capture through channel spin $S=2$, with any combination of d-and g-capture, lie on the drawn ellipse; also the point for g-capture through channel spin $S=1$ is indicated. All solutions within the bold lines can be considered as physically meaningful. The pattern of 66 points where $\chi^{2}$ values have been calculated is indicated.

The values of the $x_{j}$ are varied in steps $\Delta\left(\operatorname{arctg} x_{j}\right)=10^{\circ}$ and in smaller steps, $2^{\circ}$, in the neighbourhood of a minimum of $\chi^{2}$. No minimum will be missed in this way.

In the following, one example will be presented in some detail. At the $1845 \mathrm{keV}$ resonance, the resonance spin $J$ has to be determined by measurements on a $J-2-0$ cascade. The results of the measurements performed in the geometries I, II, V, VI and of the angular distribution 1 are shown in fig. 3. The curves represent the best fits for the angular dependence $W(\theta) \propto 1+A_{2} P_{2}(\cos \theta)+A_{4} P_{4}(\cos \theta)$. The $A_{2}$ and 
TABLE

Legendre polynomial coefficients of measured

\begin{tabular}{|c|c|c|c|c|c|c|}
\hline \multirow{2}{*}{$\underset{(\mathrm{keV})}{E_{\mathrm{p}}}$} & \multirow{2}{*}{$\begin{array}{c}E_{\gamma} \\
(\mathrm{MeV})\end{array}$} & \multirow{2}{*}{$\begin{array}{l}\text { Levels in the decay } \\
(\mathrm{MeV})\end{array}$} & \multicolumn{2}{|c|}{ Angular distribution 1} & \multicolumn{2}{|c|}{ Angular distribution 2} \\
\hline & & & $A_{2}$ & $A_{4}$ & $A_{2}$ & $A_{4}$ \\
\hline 733 & $\begin{array}{l}9.22 \\
7.24-1.98 \\
4.77-4.45\end{array}$ & $\begin{array}{l}9.22 \rightarrow 0 \\
9.22 \rightarrow 1.98 \rightarrow 0 \\
9.22 \rightarrow 4.45 \rightarrow 0\end{array}$ & $\begin{array}{l}-0.04 \pm 0.02 \\
+0.02 \pm 0.02\end{array}$ & $\begin{array}{l}0.00 \pm 0.02 \\
0.00 \pm 0.00\end{array}$ & & \\
\hline 860 & $\begin{array}{l}7.36-1.98 \\
5.15-2.21 \\
5.15-1.98 \\
5.15-4.19 \\
4.37-0.78 \\
3.43 \\
1.00 \\
2.21 \\
1.98\end{array}$ & $\begin{array}{l}9.34 \rightarrow 1.98 \rightarrow 0 \\
9.34 \rightarrow 4.19 \rightarrow 1.98 \\
9.34 \rightarrow 4.19 \rightarrow 1.98 \rightarrow 0 \\
9.34 \rightarrow 4.19 \rightarrow 0 \\
9.34 \rightarrow 4.97 \rightarrow 4.19 \\
9.34 \rightarrow 5.91 \\
5.19 \rightarrow 4.19 \\
4.19 \rightarrow 1.98 \\
1.98 \rightarrow 0\end{array}$ & $\begin{array}{l}-0.29 \pm 0.07 \\
-0.14 \pm 0.06 \\
+0.36 \pm 0.14 \\
-0.14 \pm 0.02 \\
+0.22 \pm 0.02\end{array}$ & $\begin{array}{r}-0.01 \pm 0.07 \\
-0.04 \pm 0.06 \\
-0.04 \pm 0.14 \\
0.00 \pm 0.02 \\
+0.01 \pm 0.02\end{array}$ & $+0.07 \pm 0.04$ & $-0.01 \pm 0.04$ \\
\hline 1097 & $\begin{array}{l}5.38-2.21 \\
5.38-1.98 \\
4.38-1.00 \\
4.38-2.21 \\
0.76 \\
3.66 \\
3.33 \\
1.98 \\
2.21\end{array}$ & $\begin{array}{l}9.57 \rightarrow 4.19 \rightarrow 1.98 \\
9.57 \rightarrow 4.19 \rightarrow 1.98 \rightarrow 0 \\
9.57 \rightarrow 5.19 \rightarrow 4.19 \\
9.57 \rightarrow 5.19 \rightarrow 4.19 \rightarrow 1.98 \\
5.19 \rightarrow 4.43 \\
9.57 \rightarrow 5.91 \\
9.57 \rightarrow 6.24 \\
1.98 \rightarrow 0 \\
4.19 \rightarrow 1.98\end{array}$ & $\begin{array}{l}-0.29 \pm 0.04 \\
-0.15 \pm 0.03 \\
-0.07 \pm 0.04 \\
+0.47 \pm 0.10 \\
-0.11 \pm 0.07 \\
+0.26 \pm 0.03 \\
-0.25 \pm 0.03\end{array}$ & $\begin{array}{r}+0.03 \pm 0.04 \\
-0.03 \pm 0.03 \\
0.00 \pm 0.04 \\
-0.10 \pm 0.10 \\
-0.05 \pm 0.07 \\
-0.07 \pm 0.03 \\
-0.02 \pm 0.03\end{array}$ & $+0.27 \pm 0.03$ & $-0.05 \pm 0.03$ \\
\hline 1118 & $\begin{array}{l}9.59 \\
7.61-1.98\end{array}$ & $\begin{array}{l}9.59 \rightarrow 0 \\
9.59 \rightarrow 1.98 \rightarrow 0\end{array}$ & $-0.02 \pm 0.04$ & $0.00 \pm 0.05$ & & \\
\hline 1129 & $\begin{array}{l}3.76(+3.87) \\
3.76-5.85\end{array}$ & $\begin{array}{l}9.61 \rightarrow 5.85(+5.85 \rightarrow 1.98) \\
9.61 \rightarrow 5.85 \rightarrow 0\end{array}$ & $-0.01 \pm 0.03$ & & $\begin{array}{r}0.00 \pm 0.03 \\
+0.01 \pm 0.03\end{array}$ & $+0.01 \pm 0.03$ \\
\hline 1761 & $\begin{array}{l}5.79-(2.45 \\
\quad+1.98) \\
3.04-5.20 \\
2.73 \\
4.45 \\
2.45 \\
1.98\end{array}$ & $\begin{aligned} 10.22 & \rightarrow 4.43 \rightarrow 1.98 \rightarrow 0 \\
10.22 & \rightarrow 7.18 \rightarrow 1.98 \\
7.18 & \rightarrow 4.45 \\
4.45 & \rightarrow 0 \\
4.43 & \rightarrow 1.98 \\
1.98 & \rightarrow 0\end{aligned}$ & $\begin{array}{l}+0.37 \pm 0.02 \\
-0.20 \pm 0.08 \\
-0.34 \pm 0.17 \\
+0.26 \pm 0.13 \\
+0.29 \pm 0.04 \\
+0.30 \pm 0.03\end{array}$ & $\begin{array}{l}-0.01 \pm 0.02 \\
+0.05 \pm 0.09 \\
+0.01 \pm 0.18 \\
-0.09 \pm 0.14 \\
-0.06 \pm 0.04 \\
-0.06 \pm 0.03\end{array}$ & $-0.14 \pm 0.14$ & \\
\hline 1845 & $\begin{array}{l}8.32-1.98 \\
4.45\end{array}$ & $\begin{aligned} 10.32 & \rightarrow 1.98 \rightarrow 0 \\
4.45 & \rightarrow 0\end{aligned}$ & $\begin{array}{l}+0.37 \pm 0.02 \\
+0.31 \pm 0.15\end{array}$ & $-0.09 \pm 0.02$ & & \\
\hline 2595 & $\begin{array}{l}9.05-1.98 \\
6.60-2.45 \\
1.76\end{array}$ & $\begin{aligned} 11.03 & \rightarrow 1.98 \rightarrow 0 \\
11.03 \rightarrow 4.43 \rightarrow 1.98 & \\
1.76 & \rightarrow 0\left({ }^{35} \mathrm{Cl}\right)\end{aligned}$ & $\begin{array}{c}-0.13 \pm 0.02 \\
-0.08 \pm 0.02 \\
-0.118 \pm 0.012\end{array}$ & $\begin{array}{l}-0.02 \pm 0.02 \\
-0.02 \pm 0.02 \\
-0.323 \pm 0.013\end{array}$ & $+0.05 \pm 0.03$ & $+0.18 \pm 0.03$ \\
\hline
\end{tabular}

a) Actually this number relates to the sum of the geometries I and II.

$A_{4}$ coefficients are displayed in table 3. In fig. 4 , the values of $\chi^{2}$ are given for four values of $J$ as a function of the radiation mixing ratio $x$ of the $10.30 \rightarrow 1.98 \mathrm{MeV}$ transition. These values of $\chi^{2}$ are minimized with respect to the occupation probabilities, which simply means that for each trial value of $x$ the smallest of 21 or 66 values of $\chi^{2}$ is plotted. Only for $J=4$ a solution is found with $\chi^{2}$ below the $0.1 \%$ probability 
angular distributions and correlations

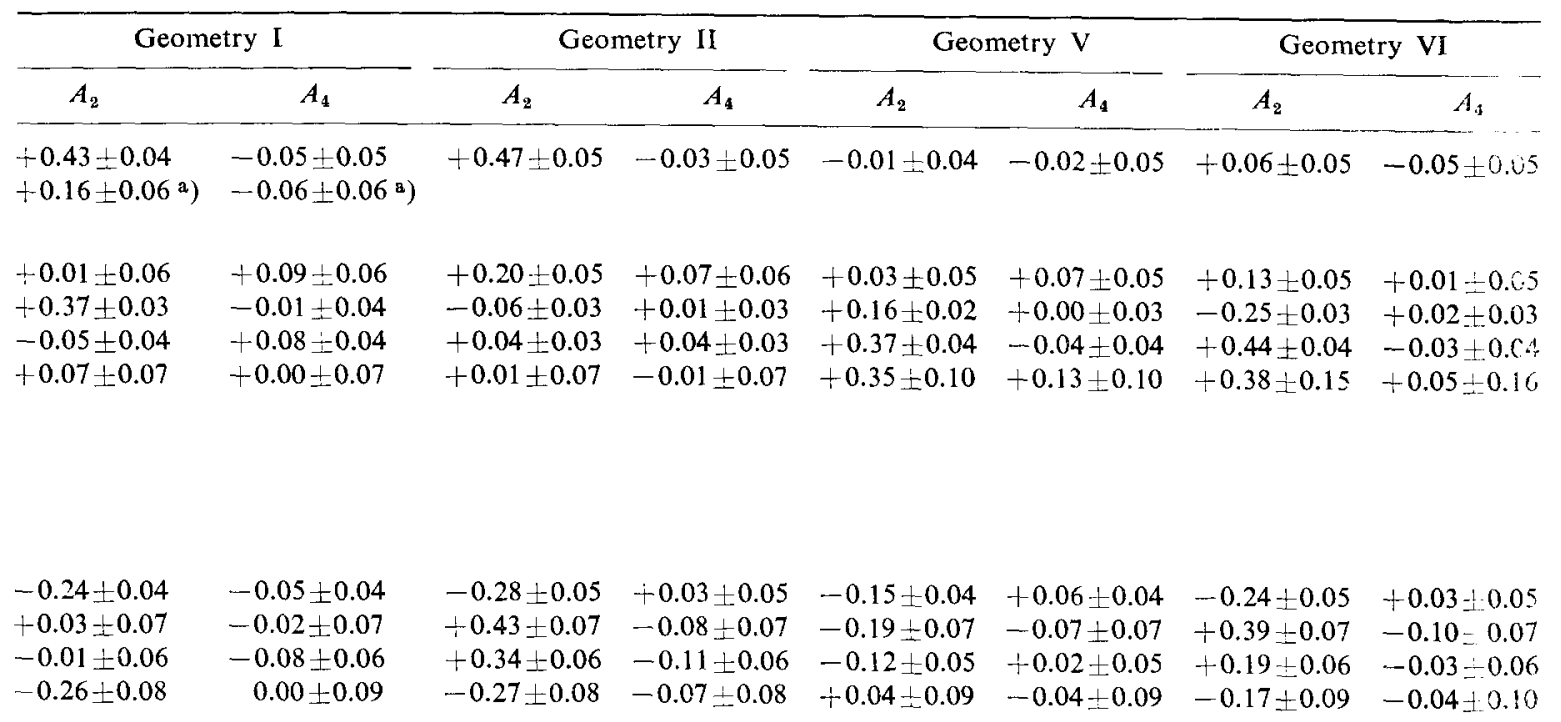

$$
\begin{aligned}
& \left.\left.+0.20 \pm 0.06^{a}\right) \quad-0.04 \pm 0.06^{a}\right) \\
& -0.28 \pm 0.05 \text { a) } \quad-0.03 \pm 0.05 a) \\
& +0.16 \pm 0.03+0.01 \pm 0.03 \quad+0.06 \pm 0.03 \quad-0.01 \pm 0.03+0.54 \pm 0.03 \quad+0.01 \pm 0.03 \quad+0.49 \pm 0.03 \quad-0.12 \pm 0.03 \\
& +0.18 \pm 0.07 \quad-0.05 \pm 0.07 \quad+0.23 \pm 0.05 \quad-0.06 \pm 0.05 \quad+0.56 \pm 0.04 \quad-0.12 \pm 0.04 \quad+0.55 \pm 0.04 \quad-0.02 \pm 0.04 \\
& -0.17 \pm 0.03+0.04 \pm 0.03+0.05 \pm 0.03+0.29 \pm 0.03-0.19 \pm 0.03+0.01 \pm 0.03+0.01+0.03+0.17 \pm 0.03 \\
& +0.04 \pm 0.05+0.02 \pm 0.05+0.20 \pm 0.04+0.11 \pm 0.04-0.07 \pm 0.04+0.04 \pm 0.04+0.06 \pm 0.04+0.14 \pm 0.04
\end{aligned}
$$

limit. The best fit is obtained for $x=0$. In fig. 5 a network of 66 points is indicated, being the coordinates of the occupation probabilities for which the $\chi^{2}$ values were computed. At the end of the program, the values of $\chi^{2}$ have been minimized with respect to the radiation mixing ratio $x$ in each of the 66 points.

For the computation of the best values and of the errors in the parameters $p(m)$ 
and the mixing ratio $x$, the assumption is made that $\chi^{2}$ is a quadratic function of these variables in the neighbourhood of the minimum. The error region corresponding to the statistical standard deviation can be found by intersection of the unnormalized $\chi^{2}$ function with the surface $\chi^{2}=\chi_{\min }^{2}+1$. It can be shown that the calculation of errors in this way is equivalent to methods where the least-squares equations are explicitly linearized and the errors in the parameters are found by propagation of errors ${ }^{22}$ ). In one dimension the error determination can simply be performed graphically. A problem of more dimensions can always be reduced to one of fewer dimensions by minimizing with respect to the remaining parameters. The best value and error of the radiation mixing ratio $x$ can thus be obtained from fitting a parabola in the region of the minimum of $\chi^{2}$; the values of $\chi_{\min }^{2}$ and $\chi_{\min }^{2}+1$ are indicated in fig. 4 . The best values and errors of the occupation probabilities $p(m)$ are found from the least-squares fit of a paraboloid to a number of the 66 values of $\chi^{2}$, calculated for the $p(m)$ values marked with dots in fig. 5. If the normalized $\chi^{2}$, i.e. $\chi_{\mathrm{n}}^{2}$, is larger than 1 , all errors are multiplied by $\chi_{\mathrm{n}}$. The error region of the occupation probabilities $p(m)$ is the shaded region in fig. 5 . The possible solutions for capture via channel spin 2 for any combination of $\mathrm{d}$ and $\mathrm{g}$ capture are also indicated. These lie on the indicated ellipse, determined by a phase relation between the $\mathrm{d}$ and $\mathrm{g}$ capture amplitudes (see ref. $\left.{ }^{9}\right)$ ). Capture via channel spin 1 and $l=4$, yields the point $p(1)=1$. All linear combinations of solutions on the ellipse and the point $p(1)=1$ can be considered as possible. Taking into account the smaller penetrability of the Coulomb barrier for high orbital momenta one obtains for the most probable solution $\varepsilon_{2}=+0.20 \pm 0.06$ and $\tau=0$, which corresponds to mainly d capture with $4 \%$ admixture of $g$ capture.

This result invalidates the statement of Nordhagen ${ }^{24}$ ) that a statistical tensor description of the alignment of the resonance level is to be preferred to the population probability description, as the latter is shorter in this case where three population parameters are needed instead of four statistical tensors. However, if higher channel spins are involved in a reaction, the tensor formalism may be preferred.

The method of analysis, presented so far, is also feasible if more than one cascade is analysed at a resonance. If two independent cascades are analysed, the $\chi^{2}$ patterns of both cascades, calculated for $p(m)$ values indicated with dots in fig. 5, can simply be added to obtain the total least-squares fit and the best values for the occupation probabilities $p(m)$. Also for multiple cascades the method can be used very well. In the case that angular correlations between different combinations of two transitions in a multiple cascade have the continuous parameters in common, all correlation measurements can be treated as so many geometries.

\subsubsection{Methods for partial analysis of angular correlations}

(a) Analysis of angular distributions. It is evident that the described methods of analysis can be lengthy. In a number of cases where angular distributions are measured, a partial analysis is possible. The intention thereof is not the elimination of spin values (though this may be possible occasionally), but elimination of large parameter 
regions. The angular distributions of $\gamma_{1}$ and $\gamma_{2}$, e.g. are described by eqs. (17) and (18) with $x_{1}, x_{2}, \rho_{20}\left(J_{1} J_{1}\right)$ and $\rho_{40}\left(J_{1} J_{1}\right)$ as parameters. If values for $x_{1}$ and $x_{2}$ are inserted, the least-squares equations for the $A_{2}$ and $A_{4}$ coefficients are linear in the statistical tensors and can be solved with a speed not easily obtained with more complicated programs. The values for $x_{1}$ and $x_{2}$ are varied in discrete steps in the program. Limiting values are simply imposed on the statistical tensors from the requirement that the population parameters of the level with spin $J_{1}$ are positive. The method is essentially a simplified version of Smith's programs ${ }^{9,22}$ ). Problems with three radiation mixtures can be worked out also in a reasonable time. The method was applied to the analysis of the $9.57 \rightarrow 5.19 \rightarrow 4.19 \rightarrow 1.98 \mathrm{MeV}$ cascade at the 1097 $\mathrm{keV}$ resonance.

(b) Extension to the analysis of triple correlations. The method described under (a) is especially suitable for the analysis of gamma transitions via high-energy bound states. These states are often excited only via one cascade. Low-energy bound states are often populated through several cascades. With a coincidence measurement on the first transition of a cascade the lower transitions of that cascade can be treated simply. We consider a three-step cascade as in fig. 2 . After proton capture and emission of $\gamma_{1}$ at the angles $\theta_{1}=\frac{1}{2} \pi, \phi_{1}=0$, the spin polarization of the nuclei in the state with spin $J_{2}$ can be described by statistical tensor components $\rho_{M N}\left(J_{2} J_{2}\right)$, with $-M \leqq N$ $\leqq+M$ and $N$ even, see eq. (19). The angular correlations of all radiation $\gamma_{i}\left(\gamma_{2}, \gamma_{3}\right.$, etc.) emitted in coincidence with $\gamma_{1}$, can then be described by a series of the form

$$
\begin{aligned}
W\left(\theta_{i}, \phi_{i}\right)=\sum_{M} \sum_{N \geqq 0, \text { even }}\left[\left|\rho_{M N}\left(J_{2} J_{2}\right)\right| / \rho_{00}\left(J_{2} J_{2}\right)\right] S_{M}^{i}(-1)^{N} \\
\times[(M-N) ! /(M+N) !]^{\frac{1}{2}} P_{M}^{N}\left(\cos \theta_{i}\right) \cos \left(N \phi_{i}\right),
\end{aligned}
$$

as follows from eqs. (21) and (22).

The factor $S_{M}^{2}$ for the case of observation of $\gamma_{2}$ follows from a comparison of eq. (26) with eq. (21). In the case of observation of subsequent radiation, one should renumber the indices in eq. (21) and insert expressions as given in eq. (22) for the intermediate unobserved radiations. For the case of observation of $\gamma_{2}$ and $\gamma_{3}$, the coefficients $S_{M}^{i}$ can be computed from the tables of Kaye et al. ${ }^{20}$ ), remembering that

$$
\begin{gathered}
S_{M}^{2}=\left(e_{2} / 4 \pi\right) \sum_{L_{2} \leqq L_{2}^{\prime}} A_{M 0}^{0}\left(J_{2} J_{3} J_{4} L_{2} L_{2}^{\prime} L_{3} L_{3} M\right) Q_{M}(2 M+1)^{\frac{1}{2}} \frac{x_{2}^{L_{2}+L_{2}^{\prime}-2 L_{2} \text { min }}}{\left(1+x_{2}^{2}\right)} \\
S_{M}^{3}=\left(e_{3} / 4 \pi\right) \sum_{L_{2}} \sum_{L_{3} \leqq L_{3}^{\prime}} A_{0 M}^{0}\left(J_{2} J_{3} J_{4} L_{2} L_{2} L_{3} L_{3}^{\prime} M\right) Q_{M}(2 M+1)^{\frac{1}{2}} \\
\times \frac{x_{2}^{2\left(L_{2}-L_{2} \text { min }\right)} x_{3}^{L_{3}+L_{3}^{\prime}-2 L_{3} \text { min }}}{\left(1+x_{2}^{2}\right)\left(1+x_{3}^{2}\right)}-
\end{gathered}
$$

which are analogous to eqs. (17a) and (18a) for the angular distributions.

The $\phi$-dependence in (26) can be eliminated, so that only the terms proportional to $\rho_{M 0}\left(J_{2} J_{2}\right)$ remain and the angular correlations can be treated as angular distribu- 
tions. In most practical cases it is sufficient to measure the geometries II and VI, where $\phi=\pi$ and $\frac{1}{2} \pi$, respectively, with the same normalization. Experimentally this is not difficult. As $\cos 2 \pi=-\cos \pi$, the term with $N=2$ is eliminated if the measurements in the geometries II and VI are added. The term with $N=4$ only rarely appears in multiple cascades, because it requires that the transitions $\gamma_{1}$ and $\gamma_{i}$ contain predominantly quadrupole radiation. Formally, terms with $N=2,4$ and 6 can be eliminated by performing and adding normalized measurements at $\phi=\frac{7}{8} \pi$ and $\frac{5}{8} \pi$.

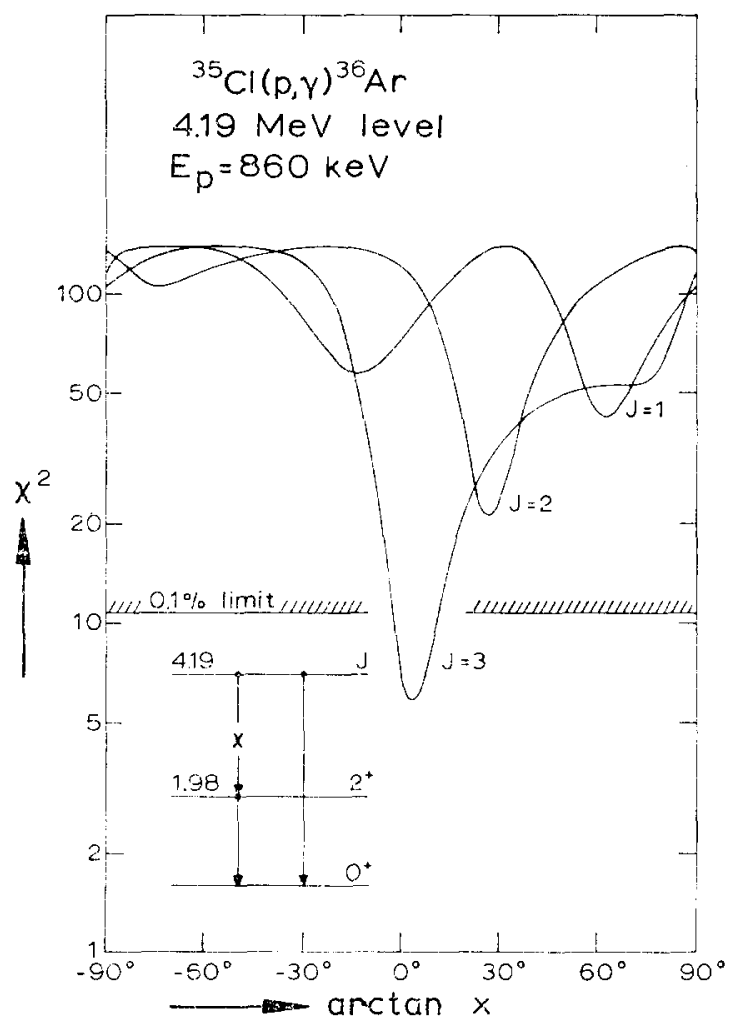

Fig. 6. Values of $\chi^{2}$ from $\gamma$-ray triple correlations of the $9.34 \rightarrow 4.19 \rightarrow 1.98 \rightarrow 0 \mathrm{MeV}$ and $9.34 \rightarrow 4.19 \rightarrow 0 \mathrm{MeV}$ cascades, in pseudo angular distribution arrangement, at the $860 \mathrm{keV}$ resonance, plotted against the radiation mixing ratio of the $2.21 \mathrm{MeV}$ transition with the spin of the $4.19 \mathrm{MeV}$ level as a parameter. For the meaning of the $0.1 \%$ probability limit, see the text.

Summarizing, one sees that it is possible to measure pseudo angular distributions in multiple cascades. In even nuclei a $2^{+} \rightarrow 0^{+}$transition, that of the last step, gives good information about the magnitude of $\rho_{20}\left(J_{2} J_{2}\right)$ and $\rho_{40}\left(J_{2} J_{2}\right)$.

As an example of this method may serve the determination of the spin of the 4.19 $\mathrm{MeV}$ level from a number of triple correlation measurements at the $860 \mathrm{keV}$ resonance. The decay of this level is indicated in an insert in fig. 6. Addition of the triple correlation measurements in coincidence with the $5.15 \mathrm{MeV}$ transition from the resonant 
state to the $4.19 \mathrm{MeV}$ level, in the geometries II and VI, gives the $A_{2}$ and $A_{4}$ coefficients:

$$
\begin{array}{ccc}
E_{\gamma} \mathrm{MeV} & A_{2} & A_{4} \\
4.19 & +0.17 \pm 0.07 & +0.02 \pm 0.07 \\
2.21 & -0.17 \pm 0.03 & +0.02 \pm 0.03 \\
1.98 & +0.20 \pm 0.02 & +0.00 \pm 0.02 .
\end{array}
$$

The $\chi^{2}$ analysis of the $A_{2}$ coefficients is displayed in fig. 6 as a function of the radiation mixing ratio of the $2.21 \mathrm{MeV}$ transition for $J_{4.19}=1,2$ and 3. Only the solution for $J=3$ reaches below the $0.1 \%$ probability limit.

\subsection{RESULTS OF ANGULAR CORRELATION MEASUREMENTS}

In this section the results of angular correlation measurements at eight resonances will be presented. Definite spin assignments are made if a $\chi_{(\min )}^{2}$ value above the $0.1 \%$ probability limit has been obtained for all $J$ possibilities but one, or in cases where otherwise M2 radiation would be involved with a strength greater than 5 Weisskopf units (henceforth denoted by W.U.). For Weisskopf estimates of radiation strengths a nuclear radius $r=r_{0} A^{\frac{1}{3}}$ with $r_{0}=1.2 \mathrm{fm}$ is used.

Tentative isospin assignments are given on the basis of isospin selection rules for $\mathrm{M} 1$ and E1 radiation. Dipole transitions are inhibited in $\Delta T=0$ transitions in selfconjugate nuclei. Experimental indications for inhibition of dipole radiation from resonance levels in the reaction ${ }^{32} \mathrm{~S}(\alpha, \gamma){ }^{36} \mathrm{Ar}$ were obtained earlier $\left.{ }^{2}\right)$. An inhibition of about a factor 10 can be expected for $\Delta T=0$ transitions. The radiation strengths of uninhibited $\Delta T=1$ transitions are expected to be of the order of $0.15 \mathrm{~W} . \mathrm{U}$. and 0.002 W.U. for $\mathrm{M} 1$ and E1 radiation, respectively, for strong transitions. These values were obtained by van der Leun ${ }^{29}$ ) as averages for a number of uninhibited transitions in different nuclei with $A=20-40$.

Assignments of predominant configurations are considered as possible in cases of strong M1 radiation, which implies conservation of orbital angular momentum $(\Delta l=0)$ in the nucleus, or in cases of strikingly selective proton capture or emission.

The decays and the strengths of the investigated resonances were reported in ref. ${ }^{4}$ ). In addition, upper limits for the intensities of some transitions to the ground and first excited states will be given in this section. At the $860 \mathrm{keV}$ resonance an additional $5 \%$ transition to the $4.43 \mathrm{MeV}$ level was observed. The spectra and the decay schemes of most resonances, to be discussed, will be presented in this section as a proof of the presence of the observed transitions. For some of the spectra, figures in ref. ${ }^{4}$ ) will be quoted.

The $A_{2}$ and $A_{4}$ coefficients of all measured angular correlations are listed in table 3.

2.4.1. The $733 \mathrm{keV}$ resonance. The single spectrum and the decay of this resonance are indicated in fig. 7. The resonance strength $(2 J+1) \Gamma_{\mathrm{p}} \Gamma_{y} / \Gamma$ is equal to $0.8 \mathrm{eV}$. The results of the angular correlation measurements are presented in table 3 . As the resonance decays $45 \%$ to the ground state, only the spin values 1,2 and 3 are considered 


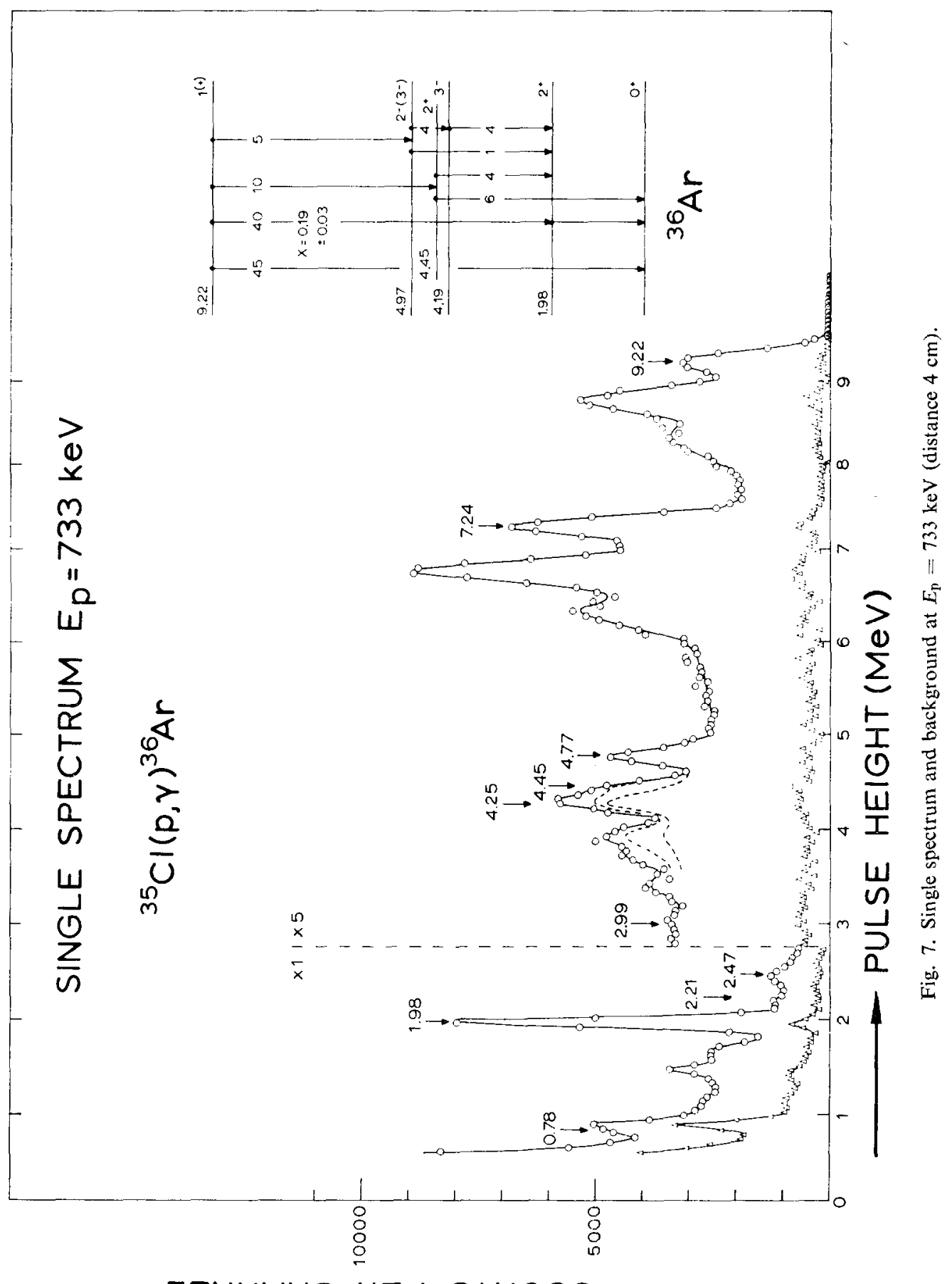

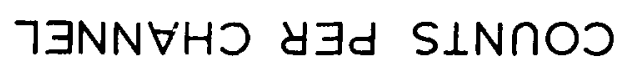


as possible for the resonance. The possibility $J=3$ is ruled out by the isotropy of the ground-state transition, and by its strength which would amount to at least 700 W.U. for E3 radiation. For $J=1$ and 2, the triple correlations through the first excited $2^{+}$state at $1.98 \mathrm{MeV}$, and the angular distribution of the ground-state transition were fitted with a $\chi^{2}$ program. The fit for $J=2$ gives $\chi_{\mathrm{n}(\min )}^{2}=10$, well above the $0.1 \%$ limit ( 3.1 in this case). The best fit occurs for $J=1$ with $\chi_{\mathrm{n}(\min )}^{2}=0.6$, a radiation mixing ratio $x=-0.19 \pm 0.03$ for the $9.22 \rightarrow 1.98 \mathrm{MeV}$ transition and the population parameters $p(0)=0.36 \pm 0.02$ and $p(1)=0.64 \pm 0.02$.

The parity of the resonance is probably even. The formation parameters would be in agreement with pure s-capture. Moreover, odd parity would entail an M2 admixture in the transition to the $1.98 \mathrm{MeV}$ level of at least the order of one W.U., which is improbable.

The decay to the 4.45 and $4.97 \mathrm{MeV}$ levels will be treated in subsects. 2.4 .9 and 2.4.10, respectively.

Summary of the results:

$$
J_{\mathrm{res}}^{\pi}=1^{(+)}, \quad p(0)=0.36 \pm 0.02, \quad p(1)=0.64 \pm 0.02, \quad x_{7.24}=-0.19 \pm 0.03 .
$$

2.4.2. The $860 \mathrm{keV}$ resonance and the $4.19 \mathrm{MeV}$ level. The strength of the resonance is $5 \mathrm{eV}$. The single spectrum is shown in fig. 8 , together with the decay, which mainly takes place via the $4.19 \mathrm{MeV}$ level. The intensity of the transition to the 1.98 $\mathrm{MeV}$ level is only $5 \%$ of the total intensity, and the ground-state transition is weaker than $0.13 \%$. The $4.19 \mathrm{MeV}$ level decays $95 \%$ to the $1.98 \mathrm{MeV}$ level and $5 \%$ to the ground state. The triple correlation measurements of the cascade via the $4.19 \mathrm{MeV}$ level were performed with coincidence spectra. These were taken at four angles for each geometry and in four geometries, with the channel set at the $5.15 \mathrm{MeV}$ line of the $9.34 \rightarrow 4.19 \mathrm{MeV}$ transition. The intensities of the $4.19,2.21$ and $1.98 \mathrm{MeV}$ lines were deduced from the spectra. The $4.19 \mathrm{MeV}$ line is partly obscured by coincident summing of the 1.98 and $2.21 \mathrm{MeV}$ lines. Corrections for this effect make the results of the correlations of the $9.34 \rightarrow 4.19 \rightarrow 0 \mathrm{MeV}$ cascade inaccurate. The results of all correlation measurements are presented in table 3 .

The spin of the $4.19 \mathrm{MeV}$ level was proven to be $J=3$, using the results from the geometries II and Vl. The method of analysis was described as an example in subsect. 2.3.2b. The spin of the resonance was investigated by analysing the triple correlations of the cascade $9.34 \rightarrow 1.98 \rightarrow 0 \mathrm{MeV}$. These measurements were hampered by coincident summing effects from the much stronger triple cascade through the 4.19 $\mathrm{MeV}$ level. Corrections for this effect increased the errors. Nevertheless the spin values $J_{\text {res }}=1$ and 4 could be exluded with a $\chi^{2}$ program (see table 4 , where the values of $\chi_{\mathrm{n}(\min )}^{2}$ are given). The spins $J_{\text {res }}=2$ and 3 both gave good fits. In order to obtain a definite choice between the possible spin combinations a combined least-squares fit to all correlation measurements through the 1.98 and $4.19 \mathrm{MeV}$ levels was performed. The only possible spin proved to be $J_{\text {res }}=3$ (see table 4 ). The best values for the 


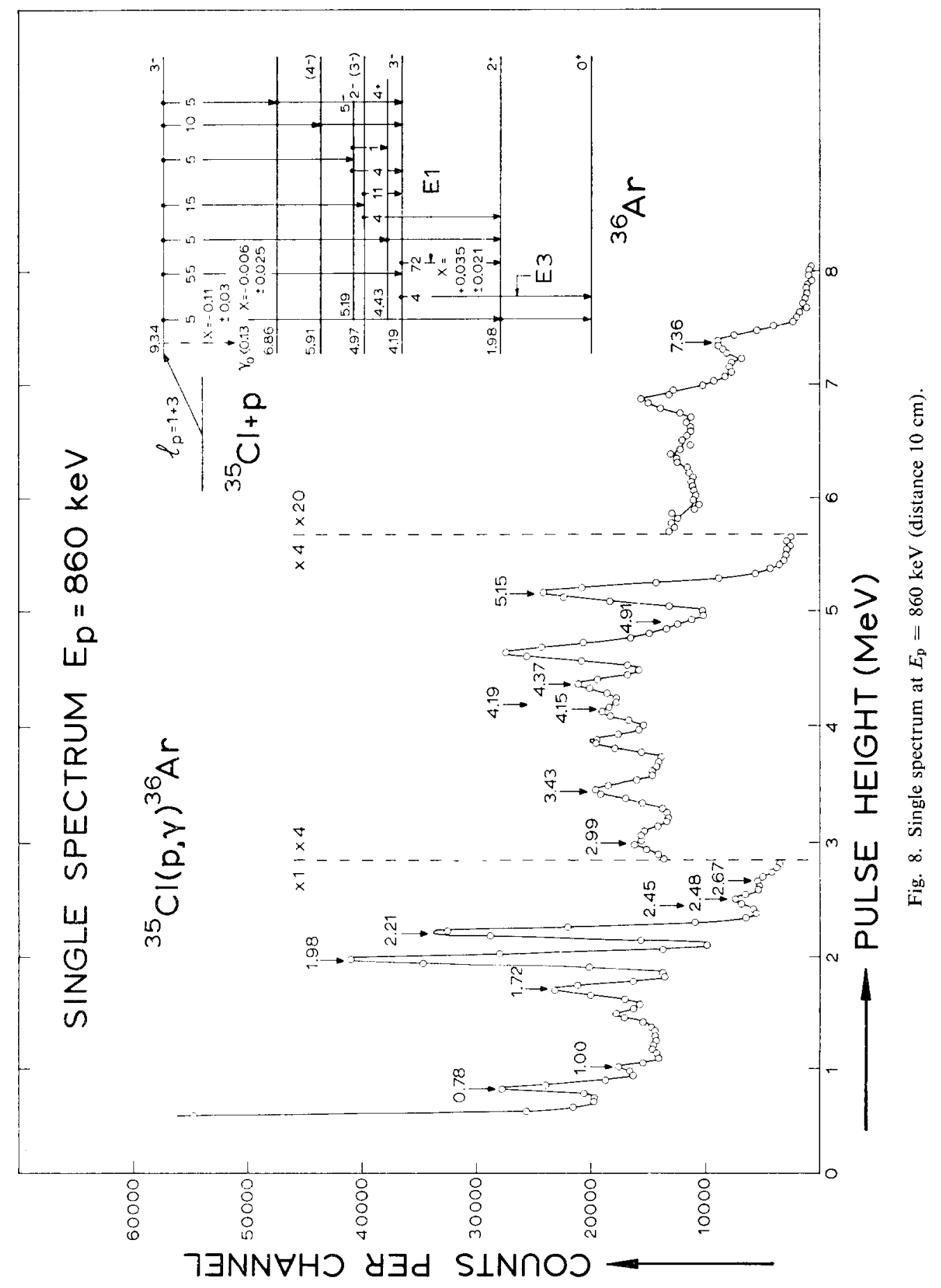


TABLE 4

\begin{tabular}{|c|c|c|c|c|c|c|}
\hline \multicolumn{7}{|c|}{ Normalized minimum $\chi^{2}$ values for the least-squares fits at the $860 \mathrm{keV}$ resonance } \\
\hline \multicolumn{3}{|c|}{$\begin{array}{l}\text { Least-squares fit for the } \\
9.34 \rightarrow 1.98 \rightarrow 0 \mathrm{MeV} \text { cascade }\end{array}$} & \multicolumn{4}{|c|}{$\begin{array}{c}\text { Total least-squares fit to all correlation } \\
\text { measurements at cascades through the } 1.98 \\
\text { and } 4.19 \mathrm{MeV} \text { levels }\end{array}$} \\
\hline$J_{\text {res }}$ & $J_{1.98}$ & $\chi_{n}^{2}(\min )$ & $J_{\text {res }}$ & $J_{4.19}$ & $J_{1.98}$ & $\chi_{\mathrm{n}^{2}}{ }_{(\min )}$ \\
\hline 1 & 2 & 4.0 & & & & \\
\hline 2 & 2 & 0.9 & 2 & 3 & 2 & 4.8 \\
\hline 3 & 2 & 1.0 & 3 & 3 & 2 & 1.5 \\
\hline 4 & 2 & 3.7 & & & & \\
\hline$\chi_{\mathrm{n}}^{2}(0.1 \%)$ & & 3.5 & $\chi_{n}{ }^{2}(0$ & & & 1.9 \\
\hline
\end{tabular}

population parameters and the radiation mixing parameters are

$$
\begin{aligned}
& p(0)=0.25 \pm 0.03, \quad p(1)=0.36 \pm 0.04, \quad p(2)=0.39 \pm 0.03 \\
& x_{7.36}=-0.11 \pm 0.03, \quad x_{5.15}=-0.006 \pm 0.025, \quad x_{2.21}=+0.035 \pm 0.021 .
\end{aligned}
$$

The odd parities of the resonance and of the $4.19 \mathrm{MeV}$ level follow from polarization measurements (see sect. 3).

The formation of the resonance is not specified completely by the population parameters. Two solutions, with 15 and $50 \% \mathrm{f}$-capture, respectively, are obtained. One can expect mainly p-capture, as the proton energy is relatively low. About $15 \% \mathrm{f}$ capture must be admitted according to the solution with $\tau=0$ and $\varepsilon_{2}=-0.43 \pm 0.07$. This amount of f-capture amounts to at least $3 \%$ of the Wigner limit.

The isospin of the resonance $T_{\text {res }}$, is predominantly one. This follows from the radiation width of the M1 transition to the $4.19 \mathrm{MeV}$ level, which amounts to at least 0.15 W.U. The resonance may be the analogue of the $2.47 \mathrm{MeV}$ level in ${ }^{36} \mathrm{Cl}$ for which van Middelkoop and Spilling ${ }^{25}$ ) recently determined $J^{\pi}=3^{-}$. Corrections for Coulomb energy and proton-neutron mass difference give a $3^{-}$level in ${ }^{36} \mathrm{Ar}$ at about $200 \mathrm{keV}$ lower than the observed excitation energy. It is interesting to compare the reduced neutron width of the $(\mathrm{d}, \mathrm{p})$ reaction leading to the $2.47 \mathrm{MeV}$ level in ${ }^{36} \mathrm{Cl}$ with the reduced proton width of the $860 \mathrm{keV}$ resonance in ${ }^{35} \mathrm{Cl}(\mathrm{p}, \gamma){ }^{36} \mathrm{Ar}$. A value $\theta_{\mathrm{n}}^{2}=0.005$ was calculated by Hoogenboom, Kashy and Buechner ${ }^{26}$ ) from the cross section of the $(\mathrm{d}, \mathrm{p})$ reaction. If $\theta_{\mathrm{p}}^{2}=\theta_{\mathrm{n}}^{2}$, one finds $\Gamma_{\mathrm{p}}=2.0 \mathrm{eV}$. Experimentally, the lower limit for $\Gamma_{\mathrm{p}}$ of the $860 \mathrm{keV}$ resonance in ${ }^{35} \mathrm{Cl}(\mathrm{p}, \gamma)^{36} \mathrm{Ar}$ is $0.7 \mathrm{eV}$. It is worth mentioning that in the $(\mathrm{d}, \mathrm{p})$ reaction a non-negligible admixture of $\mathrm{f}$-capture must be admitted to obtain a good fit to the experimental data.

The decay of the $3^{-}$level at $4.19 \mathrm{MeV}$ appears to be a good example of the isospin selection rule for $\mathrm{E} 1$ radiation. One can compare the branching ratios for $\mathrm{E} 1$ and $\mathrm{E} 3$ decay of the $3^{-}$levels at $4.19 \mathrm{MeV}$ in ${ }^{36} \mathrm{Ar}$ and at $3.82 \mathrm{MeV}$ in ${ }^{38} \mathrm{Ar}$. The $2^{+}$levels in these nuclei have about the same excitation energy, $1.98 \mathrm{MeV}$ in ${ }^{36} \mathrm{Ar}$ and 2.17 $\mathrm{MeV}$ in ${ }^{38} \mathrm{Ar}$. The branching ratio is $95: 5$ in ${ }^{36} \mathrm{Ar}$ and $99.95: 0.05$ in ${ }^{38} \mathrm{Ar}$, the latter 


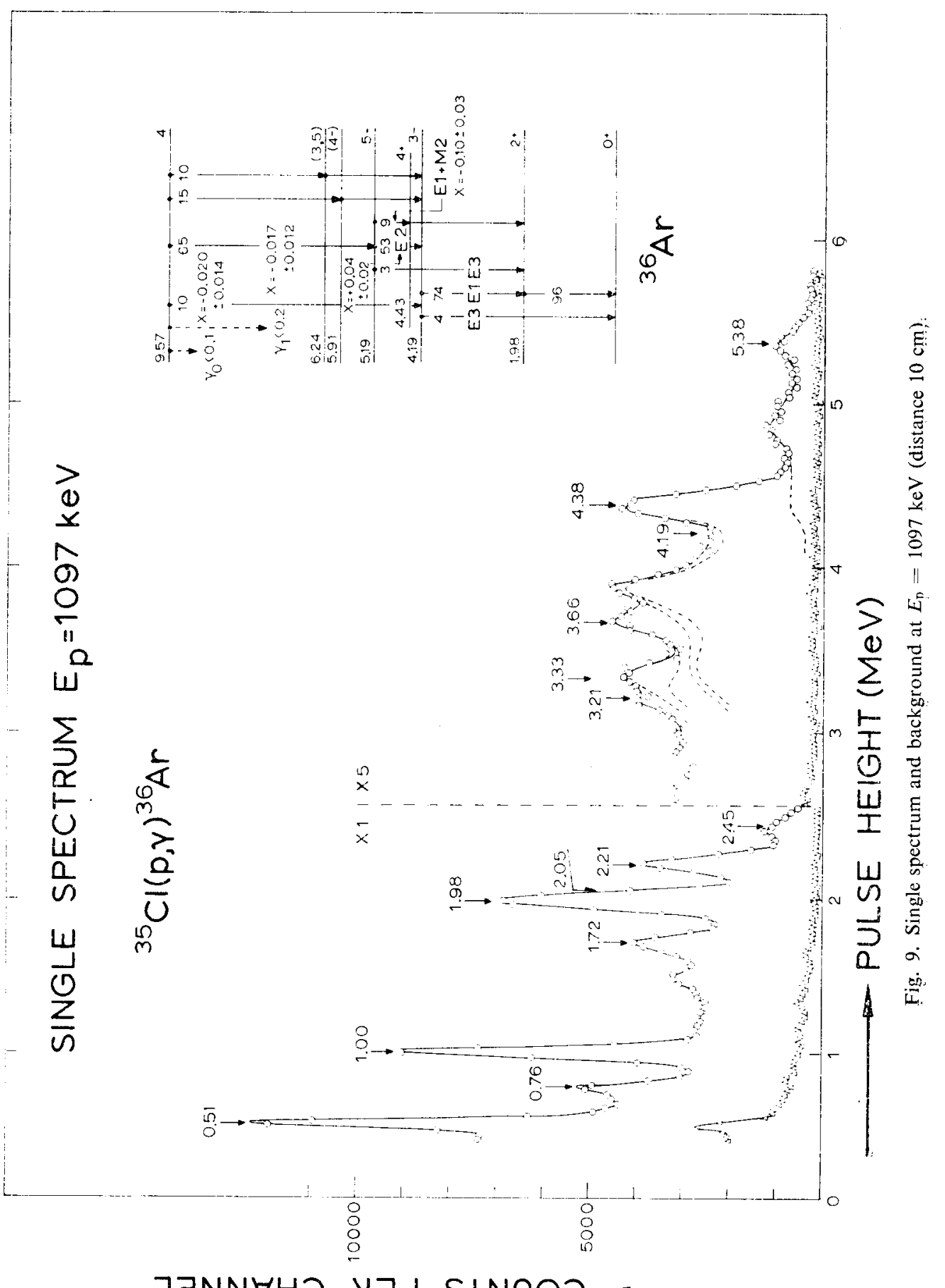

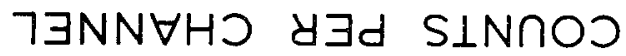


according to Robinson ${ }^{27}$ ). If the $\mathrm{E} 3$ strengths in both nuclei are taken equal, one arrives at an $\mathrm{E} 1$ hindrance factor of about 80 in ${ }^{36} \mathrm{Ar}$.

Summary of the results:

$$
\begin{aligned}
& J_{\text {res }}=3, \quad T_{\text {res }}=1, \quad J_{4.19}=3, \quad p(0)=0.25 \pm 0.03, \quad p(1)=0.36 \pm 0.04, \\
& p(2)=0.39 \pm 0.03, \quad x_{7.36}=-0.11 \pm 0.03, \quad x_{5.15}=-0.006 \pm 0.025 \\
& x_{2.21}=+0.035 \pm 0.021 .
\end{aligned}
$$

2.4.3. The $1097 \mathrm{keV}$ resonance and the $5.19 \mathrm{MeV}$ level. The decay of the $1097 \mathrm{keV}$ resonance mainly proceeds through the quadruple cascade $9.57 \rightarrow 5.19 \rightarrow 4.19 \rightarrow$ $1.98 \rightarrow 0 \mathrm{MeV}$. All observed transitions are shown, together with the single spectrum, in fig. 9. The transitions to the ground and first excited states have intensities less than 0.1 and $0.2 \%$, respectively. The resonance strength is $2.7 \mathrm{eV}$. The measured angular correlations are presented in table 3.

The spin of the resonance level was determined by triple correlation measurements at the $10 \%$ three-step cascade through the $3^{-}$level at $4.19 \mathrm{MeV}$. The triple correlations of the radiations 5.38-2.21 and 5.38-1.98 MeV were measured together, by taking coincidence spectra with a channel on the photopeak of the $5.38 \mathrm{MeV}$ transition. Combined analysis of these measurements for $J_{\text {res }}=1,2,3,4$ and 5 left $J_{\text {res }}=4$ as the only possibility (see table 5). The isospin of the resonance is probably $T_{\text {res }}=1$, as the radiation width of the $4.38 \mathrm{MeV}$ transition to the $5.19 \mathrm{MeV}$ level is large (at least 0.11 W.U. in the case of M1 radiation). From this strength one may

\begin{tabular}{|c|c|c|c|c|c|c|c|}
\hline \multicolumn{8}{|c|}{ Normalized minimum $\chi^{2}$ values for the least-squares fits at the $1097 \mathrm{keV}$ resonance } \\
\hline \multicolumn{4}{|c|}{$\begin{array}{l}\text { Least-squares fit for the } \\
9.57 \rightarrow 4.19 \rightarrow 1.98 \rightarrow 0 \mathrm{MeV} \\
\text { cascade }\end{array}$} & \multicolumn{4}{|c|}{$\begin{array}{c}\text { Total least-squares fit to all correlation } \\
\text { measurements at cascades through the } \\
5.19 \text { and } 4.19 \mathrm{MeV} \text { levels }\end{array}$} \\
\hline$J_{\text {res }}$ & $J_{4.19}$ & $J_{1.98}$ & $\chi_{n}^{2}(\min )$ & $J_{\text {res }}$ & $J_{5.19}$ & $J_{4.19}$ & $\chi_{n^{2}}(\min )$ \\
\hline 1 & 3 & 2 & $>8$ & & & & \\
\hline 2 & 3 & 2 & 2.7 & & & & \\
\hline 3 & 3 & 2 & 9.2 & 4 & 3 & 3 & 2.2 \\
\hline 4 & 3 & 2 & 1.5 & 4 & 4 & 3 & 2.8 \\
\hline 5 & 3 & 2 & $>19$ & 4 & 5 & 3 & 1.2 \\
\hline \multicolumn{3}{|c|}{$\chi_{\mathrm{n}}^{2}(0.1 \%)$} & 2.5 & $\chi_{\mathrm{n}^{2}}{ }^{2}(0.1 \%$ & & & 1.9 \\
\hline
\end{tabular}
also conclude that $\Gamma_{\mathrm{p}}>\Gamma_{\gamma}$.

TABLE 5

The spin of the $5.19 \mathrm{MeV}$ level was determined by measurements of the angular correlations in the 4.38-1.00 and 4.38-2.21 MeV transitions. These were performed by taking coincidence spectra with a channel on the photopeak of the $4.38 \mathrm{MeV}$ line and by subtracting contributions from the coincidences with the $5.38 \mathrm{MeV}$ line. The measured coincidence spectrum is shown in fig. 2 of ref. ${ }^{4}$ ), added over all angles and geometries. A preliminary search in which only the measured angular distributions 
were employed (see subsect. 2.3.2a), excluded a spin 2 for the $5.19 \mathrm{MeV}$ level, and large parts of the parameter regions for the other spin possibilities. The remaining parameter regions were investigated with a least-squares fit using all measurements at the cascades through the 5.19 and $4.19 \mathrm{MeV}$ levels (38 $A_{2}$ and $A_{4}$ coefficients). The minimum values of $\chi_{\mathrm{n}}^{2}$ are given in table 5 for $J_{5.19}=3,4$ and 5 . Only $J_{5.19}=5$ gives a good fit to all measurements. The following parameter values are obtained:

$$
x_{5.38}=-0.020 \pm 0.014, \quad x_{4.38}=-0.017 \pm 0.012, \quad x_{1.00}=+0.044 \pm 0.022 .
$$

The error regions for the population parameters are rather large. The information can be summarized in terms of statistical tensor parameters, by $\rho_{20}(J J) / \rho_{00}(J J)=$ $-0.88 \pm 0.04$. As the channel spin is much smaller than the resonance spin, this is equivalent to $\rho_{40}(J J) / \rho_{00}(J J)=+0.42 \pm 0.10$.

The parity of the resonance cannot be deduced from the formation parameters. These agree reasonably well with capture through $j_{\mathfrak{p}}=\frac{5}{2}$, with $l_{\mathrm{p}}=2$ or 3 . Competition from higher orbital momenta is improbable, since $l_{\mathrm{p}}=4$ or 5 would imply large fractions of the Wigner limit. For $l_{\mathrm{p}}=3$, the best value for the channel spin mixture ratio is $\tau=0.61 \pm 0.17$; the theoretical value for $j_{\mathrm{p}}=\frac{5}{2}$ is $\tau=0.65$.

The parity of the $J=5$ level at $5.19 \mathrm{MeV}$ was determined to be odd by polarization measurements, described in sect. 3. The decay of this level is worth some attention. The main decay proceeds through a $81 \%$ electric quadrupole transition to the 4.19 $\mathrm{MeV}$ level, whereas $14 \% \mathrm{E} 1$ and $5 \% \mathrm{E} 3$ transitions are competing. From the analysis of the angular distribution of the $0.76 \mathrm{MeV}$ line from the decay to the $4^{+}$level at $4.43 \mathrm{MeV}$ even a non-negligible $\mathrm{M} 2 / \mathrm{E} 1$ mixing must be deduced, $x_{0.76}=-0.10 \pm$ 0.03 . The isospin selection rule for $E 1$ radiation may cause this competition between the different multipolarities. Assuming that the strength of the $1.00 \mathrm{MeV}$ E2 transition is about $6 \mathrm{~W}$.U., the strength of the $3.21 \mathrm{MeV}$ E3 transition becomes 20 W.U., of the $0.76 \mathrm{MeV} \mathrm{E} 1$ component $2.10^{-5} \mathrm{~W}$.U. and of the $0.76 \mathrm{MeV} \mathrm{M} 2$ component 1.6 W.U. The strengths of the E2 and E3 transitions would be normal, whereas the E1 transition would be about 100 times slower than for non-forbidden E1 transitions. The M2 strength seems to be rather large. However, experiments performed to explain the angular distribution of the $0.76 \mathrm{MeV}$ line by possible admixtures from $4.97 \rightarrow 4.19$ or $5.91 \rightarrow 5.19 \mathrm{MeV}$ transitions gave only upper limits for these possibilities, whereas the spectrum coincident with the $0.76 \mathrm{MeV}$ line clearly shows strong enhancements of the $1.98,2.45$ and $4.38 \mathrm{MeV}$ transitions. The lifetime of the $5.19 \mathrm{MeV}$ level would be about $0.1 \mathrm{~ns}$.

The decay of the resonance to the 5.91 and $6.24 \mathrm{MeV}$ levels is treated in subsect. 2.4.11.

Summary of the results:

$$
\begin{aligned}
& J_{\text {res }}^{r}=4^{(-)}, \quad T_{\text {res }}=(1), \quad \rho_{20}(J J) / \rho_{00}(J J)=-0.88 \pm 0.04, \\
& \rho_{40}(J J) / \rho_{00}(J J)=+0.42 \pm 0.10, \quad J_{5.19}=5, \quad x_{5.38}=-0.020 \pm 0.014, \\
& x_{4.38}=-0.017 \pm 0.012, \quad x_{1.00}=+0.044 \pm 0.022, \quad x_{0.76}=-0.10+0.03 .
\end{aligned}
$$


2.4.4. The $1118 \mathrm{keV}$ resonance. The decay of this resonance, $20 \%$ to the $0^{+}$ ground state and $5 \%$ to the $4^{+}$state at $4.43 \mathrm{MeV}$ (see fig. 13), determines the resonance spin as $J=2$. A $1^{-}$or $3^{-}$assignment would entail an E3 strength of at least $80 \mathrm{~W} . \mathrm{U}$. for transitions to the $4^{+}$or $0^{+}$state, respectively. The isotropic ground-state transition corresponds to capture through $j_{\mathrm{p}}=\frac{1}{2}$. The value of the multipolarity mixing ratio of the $7.61 \mathrm{MeV}$ transition to the $2^{+}$level at $1.98 \mathrm{MeV}$ is $x=+0.8 \pm 0.2$, as deduced from the measured angular correlation in the $9.59 \rightarrow 1.98 \rightarrow 0$ cascade (see table 3). In the case of odd parity, the strength of the M2 admixture in this transition, and of the ground-state transition, would be at least 8 and 2 W.U., respectively. This is highly improbable.

The predominant decay of the resonance by E2 radiation is probably caused by the isospin selection rule for dipole radiation. An isospin $T_{\text {res }}=0$ is thus assigned.

The natural parity and isospin assignments to the resonance are confirmed by observation of $\alpha$-particles at this resonance from the ${ }^{35} \mathrm{Cl}\left(\mathrm{p}, \alpha_{0}\right){ }^{32} \mathrm{~S}$ reaction by Bošnjakovič ${ }^{28}$ ).

Summary of the results:

$J_{\text {res }}^{\pi}=2^{+}, \quad T_{\text {res }}=0, \quad$ formation probably through s-capture.

2.4.5. The $1129 \mathrm{keV}$ resonance and the $5.85 \mathrm{MeV}$ level. The weak $1129 \mathrm{keV}$ resonance decays $100 \%$ to the $5.85 \mathrm{MeV}$ level (see fig. 13), which in turn decays $93 \%$ to the ground state and $7 \%$ to the $1.98 \mathrm{MeV}$ level. Upper limits for the decay of the resonance to the ground and first excited states are $2 \%$ of the total intensity. The single spectrum is shown in fig. 6 of an earlier paper ${ }^{4}$ ). The resonance strength is $0.3 \mathrm{eV}$.

The angular distributions for radiation to and from the $5.85 \mathrm{MeV}$ level are isotropic (see table 3 ). This excludes $J_{\text {res }} \geqq 3$, as with any spin value for the intermediate 5.85 $\mathrm{MeV}$ level $\chi^{2}$ values are obtained, which are higher than the $0.1 \%$ limit. With the apparent isotropic formation little possibilities are left to obtain definite evidence for the spins involved. Because of intensity reasons only the triple correlation of the $9.61 \rightarrow 5.85 \rightarrow 0 \mathrm{MeV}$ cascade could be measured.

If $J_{\text {res }}=1$ or 2 , the $100 \%$ decay of the resonance to the $5.85 \mathrm{MeV}$ level is very unusual, as decays to the lower $0^{+}, 2^{+}, 2^{-}$and $3^{-}$states would be energetically favoured. The radiation strengths of the transitions to the ground and first excited states would be very small, compared with average values for E1 and M1 transitions.

If $J_{\text {res }}=0$, the angular distributions are isotropic, and a ground-state transition is forbidden. The angular correlation in the $9.61 \rightarrow 5.85 \rightarrow 0 \mathrm{MeV}$ cascade (see table 3 ) fits well to the spin combination $0-1-0\left(A_{2_{\mathrm{th}}}=-0.32, A_{4_{\mathrm{th}}}=0\right)$, whereas $0-2-0$ is excluded.

A spin value $J_{5.85}=1$ also gives a natural explanation of the entire spectrum. The $5.85 \mathrm{MeV}$ level would be the lowest $J=1$ level in ${ }^{36} \mathrm{Ar}$; the $3.76 \mathrm{MeV}$ line from the resonance decay to the $5.85 \mathrm{MeV}$ level would be the most probable dipole transition, having the highest possible energy.

The isospin of the resonance is probably $T_{\text {res }}=1$, as the $3.76 \mathrm{MeV}$ dipole transi- 
tion to the $5.85 \mathrm{MeV}$ level has a normal strength for uninhibited dipole radiation. Odd parity for the resonance is slightly favoured because of the absence of the $7.63 \mathrm{MeV}$ transition to the $1.98 \mathrm{MeV}$ level, which is most easily explained by the assumption of $\mathrm{M} 2$ radiation.

Summary of the results: no definite conclusions;

$$
J_{\text {res }}=(0), \quad T_{\text {res }}=1, \quad J_{5.85}=(1) \text {. }
$$

2.4.6. The $1761 \mathrm{keV}$ resonance and the $7.18 \mathrm{MeV}$ level. The single spectrum of the resonance is shown in fig. 3 of ref. ${ }^{4}$ ). The decay, see fig. 13 of the present paper, proceeds $80 \%$ to the $4^{+}$level at $4.43 \mathrm{MeV}$ and $20 \%$ to the $7.18 \mathrm{MeV}$ level. The intensities of the transitions to the ground and first excited states are less than $1 \%$ of the total intensity. The resonance strength is $2.1 \mathrm{eV}$. The results of the angular correlation measurements are presented in table 3.

The resonance spin is determined by angular correlation measurements of the $10.22 \rightarrow 4.43 \rightarrow 1.98 \rightarrow 0 \mathrm{MeV}$ cascade. The two triple correlations $5.79-2.45$ and $5.79-1.98 \mathrm{MeV}$ are identical, since the spins in the cascade are $J-4-2-0$ (see eq. (10) and following remarks in subsect. 2.2). As the 2.45 and $1.98 \mathrm{MeV}$ lines are close in energy, both the triple correlations have been measured together, in order to obtain better statistics. Least-squares fits were made for $J_{\text {res }}=2,3,4,5$ and 6 . The results from the least-squares fits are shown in table 6 , with the radiation mixing ratios and $l_{\mathrm{p}}$ values needed for the interpretation of the population parameters.

TABLE 6

Normalized minimum $\chi^{2}$ values and mixing ratios obtained from the least-squares fits of the angular correlations in the $10.22 \rightarrow 4.43 \rightarrow 1.98 \rightarrow 0 \mathrm{MeV}$ cascade, for different values of the resonance spin

\begin{tabular}{ccc}
\hline$J_{\text {res }}$ & $\chi_{\mathrm{n}}{ }^{2}(\min )$ & $x_{5.79}$ \\
\hline 2 & $>12$ & 0 \\
3 & 3.8 & $+0.48 \pm 0.08$ \\
4 & 2.4 & $-0.09 \pm 0.05$ \\
5 & 4.3 & $-0.46 \pm 0.08$ \\
6 & 24 & 0 \\
\hline$\chi_{\mathrm{n}^{2}(0.1 \%)}$ & 3.5 & \\
\hline
\end{tabular}

Only for $J_{\text {res }}=4$ one obtains a $\chi^{2}$ value below the $0.1 \%$ probability limit. The discrimination from the possibilities $J_{\text {res }}^{\pi}=3^{+}$or $5^{+}$is not very good as the $\chi_{n}^{2}$ for $J_{\text {res }}=4$ is high. Further arguments make the alternatives still more improbable, however. The possibility $J_{\text {res }}=5$ can be discarded because of the analysis of the angular distributions of the $3.04,2.73$ and $5.20 \mathrm{MeV}$ transitions to and from the $7.18 \mathrm{MeV}$ level. No $5 \rightarrow J_{7.18} \rightarrow 2$ cascade fits these angular distributions. The possibility $J_{\text {res }}^{\pi}=3^{+}$is also unlikely because of the absence of the $8.24 \mathrm{MeV}$ transition to the $1.98 \mathrm{MeV}$ level. The reasoning is as follows. The large value of the radiation mixing parameter of the $5.79 \mathrm{MeV}$ transition entails E2 radiation with a strength of at least one W.U., so that most probably $\Gamma_{\mathrm{p}}>\Gamma_{\gamma}$ holds. The strength of the $8.24 \mathrm{MeV}$ transition, measured to be less than $1 \%$ of the total intensity, would then be at most $2 \times 10^{-4}$ W.U. for M1 
radiation. This is slower than the slowest $M 1$ transition in the s-d shell observed up to now (see ref. $\left.{ }^{29}\right)$ ).

One can thus conclude that $J_{\text {res }}=4$, with a radiation mixing ratio $x_{5.79}=-0.09$ \pm 0.05 . The occupation numbers are not well determined because of lack of information on the statistical tensor component $\rho_{60}(J J)$. The information can be summarized by $\rho_{20}(J J) / \rho_{00}(J J)=-0.78 \pm 0.03$ or, which gives equivalent information, $\rho_{40}(J J) /$ $\rho_{00}(J J)=+0.14 \pm 0.07$. For odd parity, a good fit can be obtained for f-capture only, with a channel spin mixture $\tau=0.37 \pm 0.08$. This is close to the $j-j$ coupling limit for $j_{\mathrm{p}}=\frac{7}{2}, \tau=0.36$. Capture through $j_{\mathrm{p}}=\frac{5}{2}$ would give $\tau=0.65$. For even parity, a mixture of $\mathrm{d}$ - and g-capture must be considered. A continuum of solutions exists. The solution with the smallest amount of g-capture occurs for $\tau=0, \varepsilon_{2}=$ $+0.32 \pm 0.11$.

The $7.18 \mathrm{MeV}$ level is populated by the $J=4$ resonance and decays to the two lowest $2^{+}$levels. The spin values 2,3 and 4 have to be considered therefore. In the cases $J_{7,18}=2$ and 4 either the $3.04 \mathrm{MeV}$ transition to or the 2.73 and $5.20 \mathrm{MeV}$ transitions from the $7.18 \mathrm{MeV}$ level have a pure quadrupole character. These possibilities can be easily excluded with the measured angular distributions of the 3.04, 2.73 and $5.20 \mathrm{MeV}$ gamma rays and the statistical tensors obtained earlier (the values of $\chi^{2}$ are 28 and 15 for $J=2$ and 4 , respectively). For $J_{7.18}=3$, good fits are obtained for several values of the radiation mixtures. The value of the radiation mixing ratio of the $3.04 \mathrm{MeV}$ transition is $x_{3.04}=-0.03 \pm 0.14$ or +3.2 . The last value is not very probable as it would entail a strength of at least $30 \mathrm{~W}$.U. for a quadrupole component.

The even parity of the $J=4$ level at $4.43 \mathrm{MeV}$ has been determined by polarization measurements at this resonance (see sect. 3 ). These measurements also slightly favour odd parity for the resonance and even parity for the $7.18 \mathrm{MeV}$ level.

Summary of the results:

$$
\begin{aligned}
& J_{\text {res }}=4, \quad J_{7.18}=3, \quad x_{5.79}=-0.09 \pm 0.05 \text { and probably } x_{3.04}=-0.03 \pm 0.14 \\
& \rho_{20}(J J) i \rho_{00}(J J)=-0.78 \pm 0.03, \quad \rho_{40}(J J) / \rho_{00}(J J)=+0.14 \pm 0.07
\end{aligned}
$$

2.4.7. The $1845 \mathrm{keV}$ resonance. The single spectrum, a coincidence spectrum and the decay of the resonance are indicated in fig. 10 . The decay mainly proceeds through a transition to the $2^{+}$level at $1.98 \mathrm{MeV}$. The resonance strength is $1.4 \mathrm{eV}$. The results of the angular correlation measurements are shown in table 3 . The analysis has been treated as a fully illustrated example in subsect. 2.3.1. The result is that $J_{\text {res }}=4$, $x_{8.32}=0.000 \pm 0.013, p(0)=0.27 \pm 0.03, p(1)=0.41 \pm 0.03$ and $p(2)=0.32 \pm 0.03$.

The resonance has even parity as the strength $\Gamma_{\gamma}$ of the quadrupole transition to the $1.98 \mathrm{MeV}$ level is at least $0.12 \mathrm{eV}$. This would mean $20 \mathrm{~W}$.U. for M2 radiation in the case of odd parity, which is considered as highly improbable. The E2 strength of the transition is at least of the order of one W.U., indicating that $\Gamma_{\mathrm{p}}>\Gamma_{\gamma}$. The E1 transition to the $3^{-}$level at $4.19 \mathrm{MeV}$ and the M1 transition to the $4^{+}$level at 4.43 


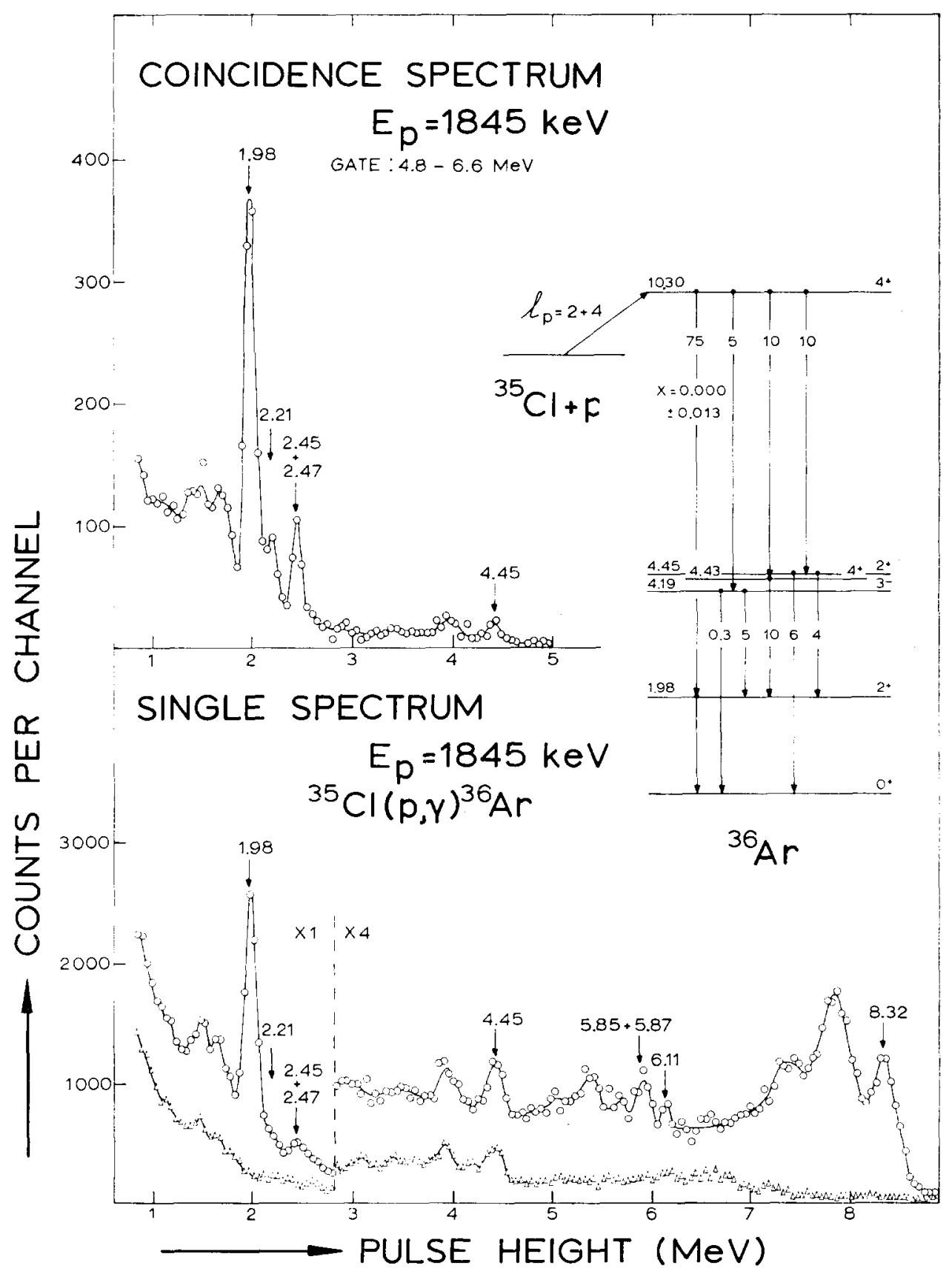

Fig. 10. Lower part: single spectrum and background at $E_{\mathrm{p}}=1845 \mathrm{keV}$ (distance $7 \mathrm{~cm}$ ), Upper part: coincidence spectrum at the same resonance (gate at $4.8-6.6 \mathrm{MeV}$, distance $4 \mathrm{~cm}$ ), showing the decays of the 4.43 and $4.45 \mathrm{MeV}$ levels. 
$\mathrm{MeV}$ are then about a factor 30 slower than normal, which is most naturally explained by the assumption that the isospin of the resonance is mainly $T=0$.

The formation of the $4^{+}$resonance can proceed through pure g-capture in the $1^{+}$ channel and through a mixture of $\mathrm{d}$ - and g-capture in the $2^{+}$channel.

There are two sets of solutions for the formation parameters $\tau$ and $\varepsilon_{2}$ that reproduce the measured particle parameters: $\tau=0, \varepsilon_{2}=+0.20 \pm 0.06$ and $\tau=0.28 \pm 0.04$, $\varepsilon_{2}=-2.1 \pm 0.6$. The first solution entails $4 \%$ g-capture and the second solution about $80 \%$. The decay to the $4.45 \mathrm{MeV}$ level is treated in subsect. 2.4.9.

Summary of the results:

$J_{\text {res }}^{\pi}=4^{+}, \quad T_{\text {res }}=0$, formation through d- + g-capture.

2.4.8. The $2595 \mathrm{keV}$ resonance, the 1.98 and $4.43 \mathrm{MeV}$ levels in ${ }^{36} \mathrm{Ar}$ and the 1.76

$\mathrm{MeV}$ level in ${ }^{35} \mathrm{Cl}$. The single spectrum and the decay of the strong $2595 \mathrm{keV}$ resonance are shown in fig. 11. The decay proceeds through the 4.43 and $1.98 \mathrm{MeV}$ levels and through proton emission to the 1.76 and $1.22 \mathrm{MeV}$ levels in ${ }^{35} \mathrm{Cl}$. The resonance strength is $17 \mathrm{eV}$. The results of the angular correlation measurements are presented in table 3 .

The measurements of the angular correlations in the $11.03 \rightarrow 1.98 \rightarrow 0 \mathrm{MeV}$ cascade give large $A_{4}$ terms in the geometries II and VI. This excludes $J=1$ for the 1.98 $\mathrm{MeV}$ level and for the resonance (see subsect. 2.3). The observation of large $A_{4}$ terms is very informative and permits a unique choice between many spin combinations. The arguments for this remark are given at the end of subsect. 2.1. In the $\chi^{2}$ fit, the values $J_{\text {res }}=2,3,4,5$ and $J_{1.98}=2$ and 3 were tried. The combination $J_{\text {res }}=3$ and $J_{1.98}=2$ gives $\chi_{\mathrm{n}(\min )}^{2}=0.7$, whereas for all other spin combinations the $\chi^{2}$ is higher than the $0.1 \%$ probability limit, see table 7 .

TABLE 7

\begin{tabular}{|c|c|c|c|c|c|c|}
\hline \multicolumn{7}{|c|}{ Normalized minimum $\chi^{2}$ values for the least-squares fits at the $2595 \mathrm{keV}$ resonance } \\
\hline \multicolumn{3}{|c|}{$\begin{array}{l}\text { Least-squares fit for the } \\
11.03 \rightarrow 1.98 \rightarrow 0 \mathrm{MeV} \text { cascade }\end{array}$} & \multicolumn{4}{|c|}{$\begin{array}{l}\text { Total least-squares fit for the } \\
11.03 \rightarrow 1.98 \rightarrow 0 \mathrm{MeV} \text { and } \\
11.03 \rightarrow 4.43 \rightarrow 1.98 \mathrm{MeV} \text { cascades }\end{array}$} \\
\hline$J_{\text {res }}$ & $J_{1.98}$ & $\chi_{n^{2}(\min )}$ & $J_{\text {res }}$ & $J_{4.43}$ & $J_{1.98}$ & $\left.\chi_{\mathrm{n}^{2}}{ }^{2} \min \right)$ \\
\hline 2 & 2 & 10 & 3 & 2 & 2 & 5 \\
\hline 3 & 2 & 0.7 & 3 & 3 & 2 & 10 \\
\hline 4 & 2 & 300 & 3 & 4 & 2 & 0.8 \\
\hline 2 & 3 & 9 & 3 & 5 & 2 & $>9$ \\
\hline 3 & 3 & 24 & & & & \\
\hline 4 & 3 & 37 & & & & \\
\hline 5 & 3 & $>100$ & & & & \\
\hline$\chi_{\mathrm{n}}^{2}(0.1 \%)$ & & 3.4 & ${ }^{2}(0.1 \%$ & & & 2.4 \\
\hline
\end{tabular}

Next the angular correlations in the $11.03 \rightarrow 4.43 \rightarrow 1.98 \mathrm{MeV}$ cascade were analysed for $J_{4.43}=2,3,4$ and 5 with the $p(m)$ values of the resonant state limited by 


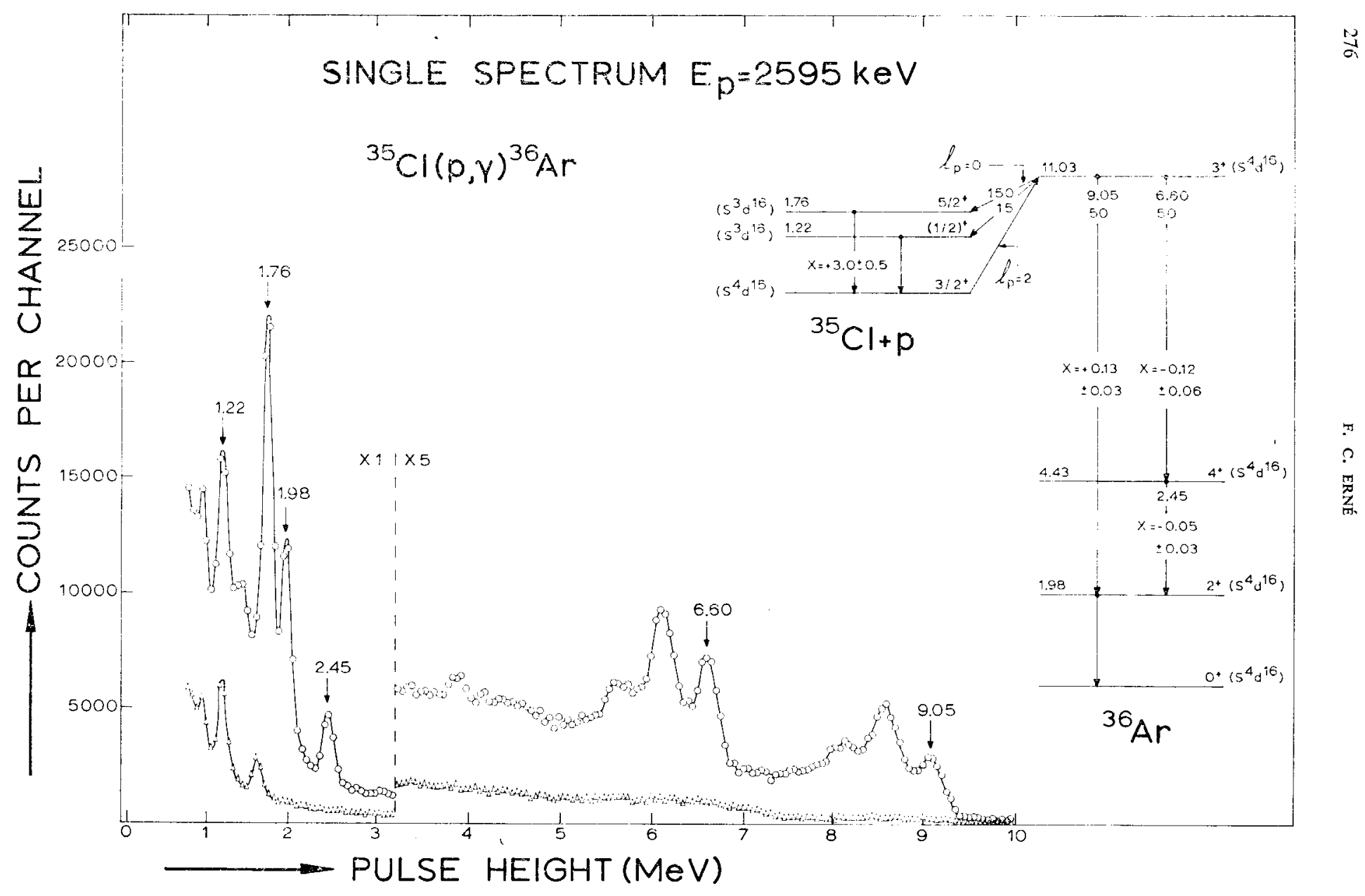

Fig. 11. Single spectrum and background at $E_{\mathbf{p}}=2595 \mathrm{keV}($ distance $7 \mathrm{~cm}$ ). 
the foregoing analysis of the $11.03 \rightarrow 1.98 \rightarrow 0 \mathrm{MeV}$ cascade. Spin 1 is excluded for the $4.43 \mathrm{MeV}$ level because of the $A_{4}$ term in the angular distribution of the $2.45 \mathrm{MeV}$ line. Only $J_{4.43}=4$ gives a $\chi^{2}$ value below the $0.1 \%$ probability limit. The results of the combined least-squares fit for both cascades investigated are displayed in table 7 . The best fit yields the following values for the parameters involved:

$$
\begin{aligned}
& x_{9.05}=+0.13 \pm 0.03, \quad x_{6.60}=-0.12 \pm 0.06, \quad x_{2.45}=-0.05 \pm 0.03 \\
& p(0)=0.041 \pm 0.018, \quad p(1)=0.288 \pm 0.026, \quad p(0)=0.670 \pm 0.023
\end{aligned}
$$

The parities of the 1.98 and $4.43 \mathrm{MeV}$ levels were determined to be even by polarization measurements at other resonances (see sect. 3 ). The parity of the resonance is also even. The population parameters fit d-capture very well, almost entirely through channel spin 2. Capture through channel spin 2 gives the $L-S$ coupling limit for $L=3, S=0$, if the $\frac{3}{2}^{+}$ground state of ${ }^{35} \mathrm{Cl}$ is described by a configuration with $L=2, S=\frac{1}{2}$. Formally admixtures from g-capture are possible, however. The least improbable solution for a mixture of $\mathrm{p}$ - and f-capture entails population parameters differing by more than three times the errors from the values determined experimentally. Odd parity is thus very improbable. The isospin of the resonance is mainly $T=1$ as the M1 transitions to the 4.43 and $1.98 \mathrm{MeV}$ levels have strengths of at least 0.20 and 0.08 W.U., respectively.

The angular distribution of the $1.76 \mathrm{MeV}$ line from proton emission to the 1.76 $\mathrm{MeV}$ level in ${ }^{35} \mathrm{Cl}$ depends on the radiation mixing ratio $x_{1.76}$ and on the relative intensities of the competing proton angular momenta. A mixture of $j_{\mathbf{p}^{\prime}}=\frac{1}{2}, \frac{3}{2}, \frac{5}{2}$ and $\frac{7}{2}$ has to be considered, so that the problem is essentially underdetermined. However, the extreme value of the $A_{4}$ coefficient observed in the angular distribution limits the proton angular momenta to at least $90 \% j_{\mathrm{p}^{\prime}}=\frac{1}{2}$ and less than $10 \%$ of any combination of $j_{\mathrm{p}^{\prime}}=\frac{3}{2}, \frac{5}{2}$ and $\frac{7}{2}$. The best fit is obtained for $j_{\mathrm{p}^{\prime}}=\frac{1}{2}$ only. The $j_{\mathrm{p}^{\prime}}=\frac{1}{2}$ value agrees well with emission through $l_{\mathrm{p}^{\prime}}=0$. Taking into account the $10 \%$ uncertainty in $j_{\mathrm{p}}$, the value of the radiation mixing ratio is $x_{1.76}=+3.0 \pm 0.5$. This value agrees well with one of the two solutions found by Hazewindus ${ }^{30}$ ) from angular correlations in the ${ }^{34} \mathrm{~S}(\mathrm{p}, \gamma){ }^{35} \mathrm{Cl}$ reaction, $x_{1.76}=+2.7 \pm 0.4$.

The lifetime of the $1.76 \mathrm{MeV}$ level in ${ }^{35} \mathrm{Cl}$, known ${ }^{43}$ ) to be $\tau_{\mathrm{m}}=0.50 \pm 0.16 \mathrm{ps}$, allows the calculation of partial lifetimes for the M1 and E2 components of the 1.76 MeV transition: $\tau_{\mathrm{m}}(\mathrm{M} 1)=5.0 \pm 2.2$ ps and $\tau_{\mathrm{m}}(\mathrm{E} 2)=0.56 \pm 0.16 \mathrm{ps}$. The M1 transition is found to be quite slow, i.e. $0.007 \mathrm{~W}$.U. The strong M1 hindrance is in qualitative agreement with shell-model calculations by Glaudemans, Wiechers and Brussaard ${ }^{3}$ ), who interpreted the 1.22 and $1.76 \mathrm{MeV}$ levels as arising from the excitation of one nucleon from the $2 s_{\frac{1}{2}}$ shell into the $1 \mathrm{~d}_{\frac{3}{2}}$ shell, whereas in the ground state of ${ }^{35} \mathrm{Cl}$ all extra-core nucleons are in the $1 \mathrm{~d}_{\frac{3}{2}}$ shell. According to this interpretation the M1 transitions to the ground state are $l$-forbidden. In fig. 11 less specific configurations are indicated for the levels involved, as these can be inferred from the present experiments. In order to obtain $l$-forbiddenness, only the total number of nucleons in the 
$2 \mathrm{~s}$ and $1 \mathrm{~d}$ orbits with a closed-shell ${ }^{16} \mathrm{O}$ core has to be considered. The main configurations $\mathrm{s}^{4} \mathrm{~d}^{15}, \mathrm{~s}^{3} \mathrm{~d}^{16}$ and $\mathrm{s}^{3} \mathrm{~d}^{16}$ are assigned to the ground state and the 1.22 and $1.76 \mathrm{MeV}$ levels in ${ }^{35} \mathrm{Cl}$, respectively.

Also the probable configurations of the ${ }^{36}$ Ar levels involved are indicated in fig. 11. The resonance level would have one more nucleon in the $1 \mathrm{~d}$ orbit than the ${ }^{35} \mathrm{Cl}$ ground state, in accordance with the observed d-capture. By emission of one nucleon from the $2 \mathrm{~s}$ orbit, the $1.76 \mathrm{MeV}$ level in ${ }^{35} \mathrm{Cl}$ can be populated, in agreement with the observed $j_{\mathrm{p}^{\prime}}=\frac{1}{2}$. The population of the $1.22 \mathrm{MeV}$ level in ${ }^{35} \mathrm{Cl}$ should be hindered according to the configuration assignments. Emission of a proton with $l_{p^{\prime}}=2$ from the resonant state, as required by the parity and angular momentum conservation rules, leaves the residual nucleus in a $s^{4} d^{15}$ state instead of $s^{3} d^{16}$. The excitation of the $1.22 \mathrm{MeV}$ level is in fact about 20 times weaker than the excitation of the 1.76 $\mathrm{MeV}$ level, if the different Coulomb barrier penetration factors are taken into account. The gamma transitions from the resonance to the 4.43 and $1.98 \mathrm{MeV}$ levels in ${ }^{36} \mathrm{Ar}$ are clearly not $l$-forbidden. One may thus conclude that mainly s ${ }^{4} \mathrm{~d}^{16}$ configurations describe the 4.43 and $1.98 \mathrm{MeV}$ levels.

The E2 component of the $1.76 \mathrm{MeV}$ transition in ${ }^{35} \mathrm{Cl}$ can be considered as of normal strength $12 \mathrm{~W} . \mathrm{U}$. The last figure makes a useful extrapolation possible of the graph of E2 transitions throughout the s-d shell made by the Chalk River group ${ }^{32}$ ).

Summary of the results:

$$
\begin{aligned}
& J_{\text {res }}^{\pi}=3^{+}, \quad T_{\text {res }}=1, \quad J_{4.43}=4, \quad J_{1.98}=2, \\
& x_{9.05}=+0.13 \pm 0.03, \quad x_{6.60}=-0.12 \pm 0.06, \quad x_{2.45}=-0.05 \pm 0.03, \\
& x_{1.76}\left({ }^{35} \mathrm{Cl}\right)=+3.0 \pm 0.5, \\
& p(0)=0.041 \pm 0.018, \quad p(1)=0.288 \pm 0.026, \quad p(2)=0.670 \pm 0.023,
\end{aligned}
$$

configuration assignments (see above).

2.4.9. The 4.45 MeV level. The $4.45 \mathrm{MeV}$ level is excited at the $733 \mathrm{keV}$ resonance $\left(J^{\pi}=1^{(+)}\right)$and at the $1845 \mathrm{keV}$ resonance $\left(J^{\pi}=4^{+}\right)$with intensities as indicated in figs. 7 and 10 . The level decays $38 \%$ to the $2^{+}$level at $1.98 \mathrm{MeV}$ and $62 \%$ to the $0^{+}$ground state. Spin values $J_{4.45}=1,2$ and 3 are thus considered as possible.

At the $733 \mathrm{keV}$ resonance the angular correlation in the $9.22 \rightarrow 4.45 \rightarrow 0 \mathrm{MeV}$ cascade has been measured in geometry $\mathrm{I}$. The result is indicated in table 3 . With the resonance spin and the population parameters already obtained, this measurement is incompatible with a $J=3$ assignment to the $4.45 \mathrm{MeV}$ level. Pure quadrupole radiation in the $4.77 \mathrm{MeV}$ transition would give $A_{2}=-0.26$, in contrast with the experimental value $A_{2}=+0.16 \pm 0.06$. The spin values $J_{4.45}=1$ and 2 remain possible (with $x_{4.77}=+0.08 \pm 0.08$ for $J_{4.45}=2$ ).

The radiation strength of the transition to the $4.45 \mathrm{MeV}$ level at the $1845 \mathrm{keV}$ resonance excludes octupole radiation and thus determines the $J=2$ assignment to the 4.45 MeV level. The parity is also determined by the strength of the transition, which 
would amount to at least $15 \mathrm{~W}$.U. for M2 radiation. The parity of the $4.45 \mathrm{MeV}$ level is thus even. A rough measurement of the angular distribution of the $4.45 \mathrm{MeV}$ transition to the ground state at the $1845 \mathrm{keV}$ resonance gives $A_{2}=+0.31 \pm 0.15$ (table 3 ). in agreement with the expected value $A_{2}=+0.35$.

Summary of the results:

$J_{4.45}^{\pi}=2^{+}, \quad x_{4.77}=+0.08 \pm 0.08$ at the $733 \mathrm{keV}$ resonance.

2.4.10. The $4.97 \mathrm{MeV}$ level. The $4.97 \mathrm{MeV}$ level is excited at the $733 \mathrm{keV}$ resonance $\left(J^{\pi}=1^{(+)}\right)$and at the $860 \mathrm{keV}$ resonance $\left(J^{\pi}=3^{-}\right)$, with intensities as indicated in figs. 7 and 8 . It decays $75 \%$ to the $3^{-}$state at $4.19 \mathrm{MeV}$ and $25 \%$ to the $2^{+}$state at $1.98 \mathrm{MeV}$. No ground-state transition was observed. Spin values $J_{4.97}=1$, 2 and 3 are thus considered as possible.

At the $860 \mathrm{keV}$ resonance the angular distributions were measured of the $4.37 \mathrm{MeV}$ transition to the $4.97 \mathrm{MeV}$ level and of the $0.78 \mathrm{MeV}$ line from the decay of the 4.97 $\mathrm{MeV}$ level to the $4.19 \mathrm{MeV}$ level (see table 3). Moreover, the polarization of the 0.78 $\mathrm{MeV}$ line has been measured at this resonance (see table 8). The angular distribution of the $4.37 \mathrm{MeV}$ line excludes $J=1$ for the $4.97 \mathrm{MeV}$ level, as pure quadrupole radiation would give $A_{2}=+0.27 \pm 0.05$, in disagreement with the experimental value $A_{2}=-0.29$ \pm 0.07 . A $J=3$ assignment is only compatible with a radiation mixing ratio $x_{4.37}=+2.2 \pm 0.6$. Even parity is then impossible as the M2 component in the 4.37 $\mathrm{MeV}$ transition would have a strength of at least $300 \mathrm{~W}$.U. Odd parity is improbable because of several reasons. In the first place the E2 component of the $4.37 \mathrm{MeV}$ line would have a strength of at least $10 \mathrm{~W}$.U., which is high compared to the average value of 0.4 W.U. mentioned by van der Leun for high-energy E2 transitions ${ }^{29}$ ). Furthermore, a $3^{-}$assignment to the $4.97 \mathrm{MeV}$ level would entail a $1^{-}$assignment to the $733 \mathrm{keV}$ resonance, as this resonance would then decay to the $4.97 \mathrm{MeV}$ level by an E2 transition. Odd parity was argued to be improbable for the $733 \mathrm{keV}$ resonance (see subsect. 2.4.1). The measurements of the angular distribution and the polarization of the $0.78 \mathrm{MeV}$ line give another argument against the $3^{-}$assignment to the $4.97 \mathrm{MeV}$ level. These are compatible only with a radiation mixture $x_{0.78}=$ $-1.9_{-0.4}^{+0.6}$. This large value of the radiation mixing ratio combined with the weak competition of the $2.99 \mathrm{MeV}$ transition to the $1.98 \mathrm{MeV}$ level can hardly be explained if $J^{\pi}(4.97)=3^{-}$.

The remaining possibilities are $J_{4.97}=2^{ \pm}$. The even parity prevents a good fit to the polarization measurements. For odd parity, there remain several possibilities for the radiation mixing parameters. The values closest to dipole are $x_{4.37}=+0.10 \pm 0.07$ and $x_{0.78}=+0.21 \pm 0.07$.

Summary of the results:

$J_{4.97}^{\pi}=2^{-}\left(3^{-}\right)$, with the $3^{-}$possibility highly improbable.

2.4.11. The 4.34, 4.99, 5.21, 5.88, 5.91, 6.24 and 7.35 MeV levels. Of the levels with excitation energies up to $6 \mathrm{MeV}$ the ones at 4.34, 4.99, 5.21, 5.88 and $5.91 \mathrm{MeV}$ 
were not yet considered. No definite spin and parity assignments can be made for these levels.

A spin and parity $0^{+}$can be suggested for the 4.34 and $5.21 \mathrm{MeV}$ levels. These levels are not excited in the 65 resonances reported earlier ${ }^{4}$ ); it seems to be a general phenomenon that excited states with $J^{\pi}=0^{+}$in self-conjugate doubly even nuclei are at best very weakly populated in $(p, \gamma)$ reactions, see for example investigations of the reactions ${ }^{27} \mathrm{Al}(\mathrm{p}, \gamma){ }^{28} \mathrm{Si}\left(\right.$ ref. $\left.\left.{ }^{33}\right)\right)$ and ${ }^{39} \mathrm{~K}(\mathrm{p}, \gamma)^{40} \mathrm{Ca}\left(\right.$ ref. $\left.\left.{ }^{34}\right)\right)$. Moreover, the 4.34 and 5.21 $\mathrm{MeV}{ }^{36} \mathrm{Ar}$ levels are weakly excited in the ${ }^{39} \mathrm{~K}(\mathrm{p}, \alpha)^{36} \mathrm{Ar}$ reaction ${ }^{1}$ ); this may be due to a $(2 J+1)$ dependence of the cross section for this reaction.

A tentative $2^{+}$assignment can be derived for the $4.99 \mathrm{MeV}$ level. This level mainly decays to the $0^{+}$ground state, whereas weaker transitions to the $2^{+} 1.98 \mathrm{MeV}$ level and $3^{-} 4.19 \mathrm{MeV}$ level were observed ${ }^{4}$ ).

The $5.88 \mathrm{MeV}$ level is not excited at any of the eight resonances reported in this investigation.

The $5.91 \mathrm{MeV}$ level is excited at the $J^{\pi}=3^{-}, 860 \mathrm{keV}$ resonance and at the $J=4$, $1097 \mathrm{keV}$ resonance. At both resonances the angular distributions were measured of the res. $\rightarrow 5.91$ transitions (see table 3 ). A spin value $J=4$ fits both angular distributions for unmixed radiation. A spin value $J=5$ is excluded by the angular distribution at the $860 \mathrm{keV}$ resonance. A spin value $J=3$ gives a bad fit at the $1097 \mathrm{keV}$ resonance and large mixing ratios at both resonances; these are improbable as the radiation strengths of the E2 components exceed $10 \mathrm{~W} . \mathrm{U}$. For the same reason, $J=2$ is improbable at the $1097 \mathrm{keV}$ resonance. The $5.91 \mathrm{MeV}$ level decays to the $3^{-}$ level at $4.19 \mathrm{MeV}$ only. Odd parity is thus probable for the level as otherwise decays to $2^{+}$levels might have been observed.

The $6.24 \mathrm{MeV}$ level is probably a high spin level. The level is excited at the $J=4$ $\left(E_{\mathrm{p}}=1097 \mathrm{keV}\right)$ resonance. The angular distribution of the $3.33 \mathrm{MeV}$ line from the resonance decay to this level (see table 3 ) suggests a spin value 5 or 3 if only small radiation mixing ratios are admitted. Large mixing ratios require strengths of more than $10 \mathrm{~W}$.U. for the $\mathrm{E} 2$ component. The level decays to the $3^{-}$level at $4.19 \mathrm{MeV}$ only. At the 2135 and $2231 \mathrm{keV}$ resonances (see ref. ${ }^{4}$ )) the level is excited together with the $7.35 \mathrm{MeV}$ level. The strongly anisotropic radiation at these resonances suggests that also the $7.35 \mathrm{MeV}$ level has a high spin.

Summary of the results:

$$
J_{4.34}^{\pi}=\left(0^{+}\right), \quad J_{4.99}^{\pi}=\left(2^{+}\right), \quad J_{5.21}^{\pi}=\left(0^{+}\right), \quad J_{5.91}^{\pi}=\left(4^{-}\right) .
$$

\section{Polarization Measurements}

Polarization measurements were performed at the 860,1097 and $1761 \mathrm{keV}$ resonances by the Compton-scattering method as described by Suffert, Hoogenboom and Endt ${ }^{35}$ ). The experimental arrangement is almost identical with the one recently described by Van Rinsvelt and Endt ${ }^{36}$ ). The main parts are shown in fig. 12 . The 
gamma rays are scattered by a $\mathrm{NaI}$ crystal $(5 \mathrm{~cm}$ thick and $5 \mathrm{~cm}$ in diam) below the target (with the proton beam coming in horizontally) and detected in one of the two $\mathrm{NaI}$ crystals $(10 \mathrm{~cm}$ thick and $10 \mathrm{~cm}$ in diam). The pulses from the scattering crystal and either of the detecting crystals are added in a summing circuit and stored in 100 channels of a 400-channel analyser, as was suggested by Hazewindus ${ }^{30}$ ). The analyser gate only transmits pulses for storage if (i) the pulses from the
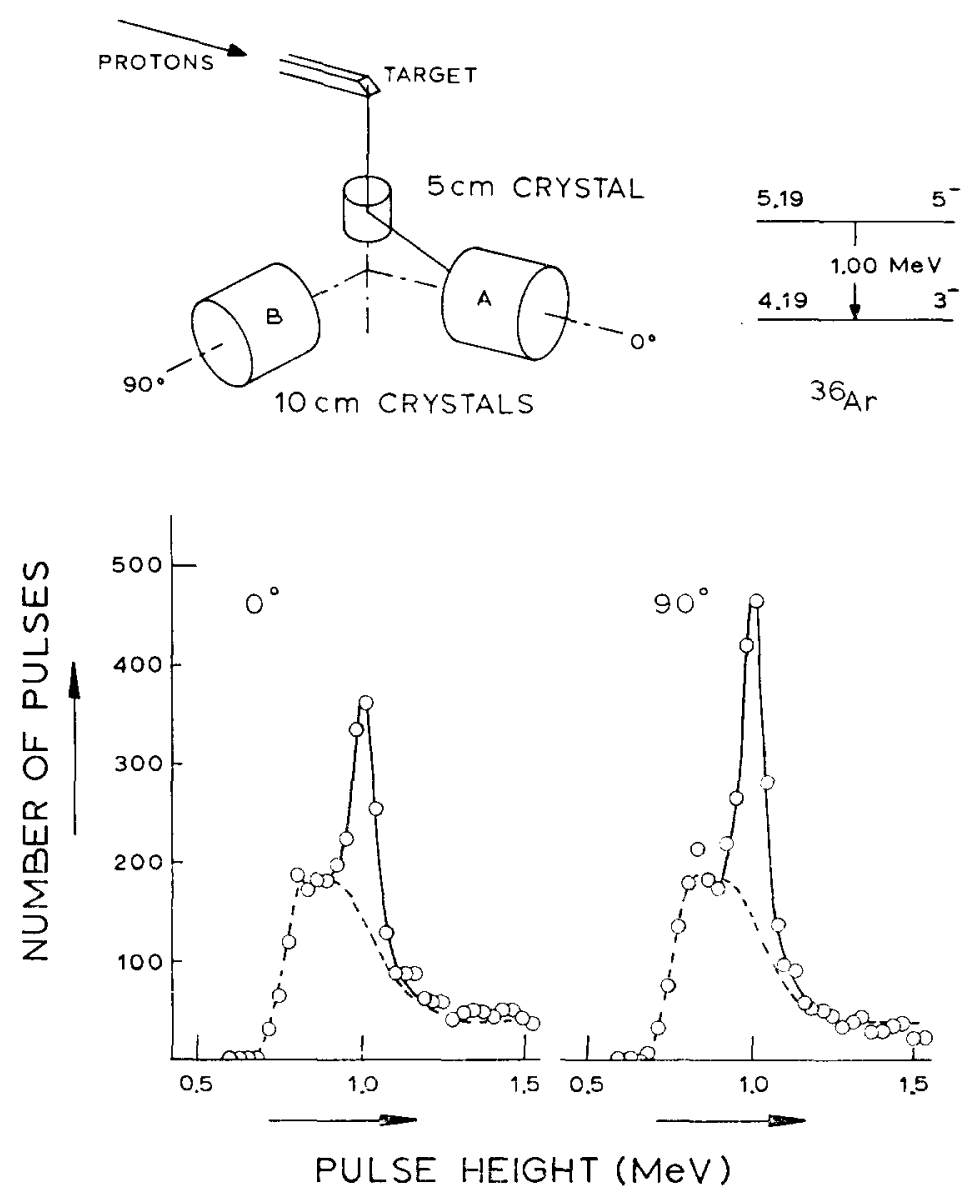

Fig. 12. Polarization measurement of the $1.00 \mathrm{MeV}$ transition from the decay of the 5.19 to the 4.19 $\mathrm{MeV}$ level at the $1097 \mathrm{keV}$ resonance. Sum-pulse spectra at $0^{\circ}$ and $90^{\circ}$. The background measurement, performed at the $1761 \mathrm{keV}$ resonance, is indicated by dashed lines. The channel setting of the $5 \mathrm{~cm}$ crystal is $0.36-0.52 \mathrm{MeV}$, of the $10 \mathrm{~cm}$ crystals $\geqq 0.38 \mathrm{MeV}$. The polarimeter used in the experiment is shown at the top.

two crystals are in coincidence $(2 \tau=2 \mu \mathrm{s})$, and (ii) the pulses from the two crystals have amplitudes in the energy range that corresponds to a single scattering of the gamma rays to be detected. With a routing system the pulses of the two detecting 
TABLE 8

Polarization measurements at three resonances in ${ }^{35} \mathrm{Cl}(\mathrm{p}, \gamma)^{36} \mathrm{Ar}$ and one resonance in ${ }^{95} \mathrm{Cl}\left(\mathrm{p}, \mathrm{p}^{\prime} \gamma\right)^{35} \mathrm{Cl}$

\begin{tabular}{|c|c|c|c|c|c|c|c|}
\hline $\begin{array}{c}E_{\gamma} \\
(\mathrm{MeV})\end{array}$ & $\begin{array}{c}\text { Transition } \\
(\mathrm{MeV})\end{array}$ & $p P_{\exp }$ & $\begin{array}{l}\text { Polari- } \\
\text { zation } \\
\text { efficiency } \\
p\end{array}$ & $\begin{array}{l}\text { Radiation } \\
\text { mixing } \\
\text { ratio } \\
x\end{array}$ & $\begin{array}{c}p P \\
\text { calculated from } \\
\text { the angular } \\
\text { distribution }\end{array}$ & $\begin{array}{c}\text { Deduced } \\
\text { radiation } \\
\text { parity }\end{array}$ & $\begin{array}{l}\text { Deduced } \\
\text { level } \\
\text { parity }\end{array}$ \\
\hline
\end{tabular}

$860 \mathrm{keV}$ resonance

\begin{tabular}{|c|c|c|c|c|c|c|c|}
\hline 0.78 & $4.97 \rightarrow 4.19$ & $-0.08 \doteq 0.04$ & 0.43 & $\left.+0.21 \pm 0.07^{\mathrm{a}}\right)$ & $=0.06 \pm 0.01$ & $\mathrm{M} 1 \div \mathrm{E} 2$ & $J \pi_{4.97}=2^{-}\left(3^{-}\right)$ \\
\hline 1.00 & $5.19 \rightarrow 4.19$ & $+0.20=0.09$ & 0.39 & $+0.04 \pm 0.02$ & $\pm 0.28=0.09$ & E2 & $J_{5.19}=5^{-}$ \\
\hline 1.98 & $1.98 \rightarrow 0$ & $\div 0.10 \div 0.02$ & 0.26 & & $\pm 0.11 \pm 0.01$ & $\mathrm{E} 2$ & $J \pi_{1.98}=2^{+}$ \\
\hline 2.21 & $4.19 \rightarrow 1.98$ & $+0.08+0.02$ & 0.25 & $+0.04 \div 0.02$ & $-0.05-0.01$ & El & $J^{\pi} 4.19=3^{-}$ \\
\hline 5.15 & $9.34 \rightarrow 4.19$ & $\div 0.09-0.03$ & 0.12 & $-0.01 \div 0.03$ & $\pm 0.06 \pm 0.01$ & $\mathrm{Ml}$ & $J^{\pi} 9.34=3^{-}$ \\
\hline
\end{tabular}

$1097 \mathrm{keV}$ resonance

$\begin{array}{llllllll}1.00 & 5.19 \rightarrow 4.19 & +0.21 \pm 0.04 & 0.39 & +0.04 \pm 0.02 & \pm 0.20 \pm 0.03 & \mathrm{E} 2 & J^{\pi}{ }_{5.19}=5^{-} \\ 1.98 \mathrm{~b}) & 1.98 \rightarrow 0 & +0.19=0.05 & 0.26 & & \pm 0.13 \pm 0.02 & \mathrm{E} 2 & J_{1.98}=2^{+} \\ 2.21 & 4.19 \rightarrow 1.98 & +0.10=0.07 & 0.25 & +0.04 \pm 0.02 & \pm 0.09 \pm 0.01 & \mathrm{E} 1 & J^{\pi}+19=3^{-}\end{array}$

$1761 \mathrm{keV}$ resonance

\begin{tabular}{|c|c|c|c|c|c|c|c|}
\hline 1.98 & $1.98 \rightarrow 0$ & $+0.09 \pm 0.03$ & 0.26 & & $0.14 \pm 0.02$ & E2 & $J x_{1.98}=2^{+}$ \\
\hline $2.45^{\mathrm{e}}$ ) & $4.43 \rightarrow 1.98$ & $+0.17+0.05$ & 0.23 & $-0.05 \doteq 0.03$ & $\pm 0.12 \pm 0.02$ & E2 & $J_{4.43}=4^{+}$ \\
\hline 2.73 & $7.18 \rightarrow 4.45$ & $-0.13 \pm 0.07$ & 0.21 & $\left.(0)^{a}\right)$ & $(\mp 0.06)$ & (M1) & $J^{\pi} 7.18=3^{(+)}$ \\
\hline 3.04 & $10.22 \rightarrow 7.18$ & $+0.03 \pm 0.06$ & 0.19 & $-0.03+0.14$ & $\therefore 0.06 \pm 0.03$ & $(\mathrm{E} 1)$ & $J \pi_{10.22}=4^{(-)}$ \\
\hline 5.79 & $10.22 \rightarrow 4.43$ & $-0.01 \div 0.05$ & 0.11 & $-0.09 \pm 0.05$ & $\mp 0.05 \pm 0.01$ & & \\
\hline
\end{tabular}

$2841 \mathrm{keV}$ resonance in ${ }^{35} \mathrm{Cl}\left(\mathrm{p}, \mathrm{p}^{\prime} \gamma^{\prime}\right)^{35} \mathrm{Cl}$
$\left.1.76^{\mathrm{e}}\right) \quad 1.76 \rightarrow 0$
$+0.050 \pm 0.015$
$0.29+3.0 \pm 0.5$
$\pm 0.037 \pm 0.010$
$\mathrm{M} 1+\mathrm{E} 2 J_{1.76}^{\pi}=\frac{5}{2}^{+}$

a) The other possible mixing ratios yield the same conclusion regarding the parity.

b) A $10 \%$ admixture of the $2.05 \mathrm{MeV}$ transition from the 6.24 to the $4.19 \mathrm{MeV}$ level was included in the $1.98 \mathrm{MeV}$ peak.

c) A $9 \%$ admixture of the $2.47 \mathrm{MeV}$ transition from the 4.45 to the $1.98 \mathrm{MeV}$ level was included in the $2.45 \mathrm{MeV}$ peak.

d) The angular distribution measurement leaves a large uncertainty in the mixing ratio.

e) The angular distribution measurement of the $1.76 \mathrm{MeV}$ line yields $A_{2}=-0.11 \pm 0.03$ and $A_{4}==-0.06 \pm 0.03$.

crystals $\mathrm{A}$ and $\mathrm{B}$ at the azimuthal positions of $0^{\circ}$ and $90^{\circ}$, respectively, are stored simultaneously each in 100 channels. These measurements are alternated every few hours with measurements where the counting system has been rotated over $90^{\circ}$, such that counter $\mathrm{A}$ is at $-90^{\circ}$ and counter $\mathrm{B}$ at $0^{\circ}$. After the measurement, the spectra at $-90^{\circ}$ and $90^{\circ}$ and the two spectra at $0^{\circ}$ are added separately. No gain stabilization was needed during the measurements due to stable temperature conditions in the measuring room. Most measurements were performed twice in order to minimize systematic errors. The eccentricity was determined by measurement of the unpolarized $1.22 \mathrm{MeV}$ line from the ${ }^{35} \mathrm{Cl}\left(\mathrm{p}, \mathrm{p}^{\prime} \gamma\right){ }^{35} \mathrm{Cl}$ reaction at the strong $2841 \mathrm{keV}$ resonance. The spin and parity of the $1.22 \mathrm{MeV}$ level in ${ }^{35} \mathrm{Cl}$ are most probably $\frac{1}{2}^{+}$. To remove all uncertainties, the angular distribution and the polarization of the $1.76 \mathrm{MeV}$ line from the ${ }^{35} \mathrm{Cl}\left(\mathrm{p}, \mathrm{p}^{\prime} \gamma\right){ }^{35} \mathrm{Cl}$ reaction were also measured at that resonance. The spin and parity of the $1.76 \mathrm{MeV}$ level in ${ }^{35} \mathrm{Cl}$ are certainly $\frac{5}{2}^{+}$. 
The polarization measurement of the $1.00 \mathrm{MeV}$ line in the spectrum of the $1097 \mathrm{keV}$ resonance requires some comment, as the discriminator channels had to be set around the $0.51 \mathrm{MeV}$ line. The measurement is shown in the lower half of fig. 12 . The possibility existed that two $0.51 \mathrm{MeV}$ pulses from pair creation would be detected. A background spectrum was taken therefore with the same channel setting at the $1761 \mathrm{keV}$ resonance. High-energy gamma rays of approximately the same energies as at the $1097 \mathrm{keV}$ resonance occurred there, but the $1.00 \mathrm{MeV}$ line was absent. The background measurement is indicated in fig. 12 by dashed lines. It shows no $1.02 \mathrm{MeV}$ line.

The results are shown in table 8 . In the first column the gamma-ray energy is indicated and in the second column the position of the transition in the decay scheme. In the third column the value of the experimentally observed effect is given. This value $p P$ is defined as $p P=\left(N_{90^{\circ}}-N_{0^{\circ}}\right) /\left(N_{90^{\circ}}+N_{0^{\circ}}\right)$. The definitions of the angles $0^{\circ}$ and $90^{\circ}$ are indicated in the upper part of fig. 12 ; they agree with the definitions customary in angular correlation measure nents and differ from the definitions by Suffert et al. ${ }^{35}$ ). However, the change is rot fundamental. The observed effect is a product of the radiation polarization $P$ and the polarization efficiency $p$ of the Compton-scattering arrangement. The values of $p$, calculated in the same way as described by Suffert et al. ${ }^{35}$ ), are indicated in column four. With the possible values of the radiation mixing ratios $x$, indicated in column five, and the experimentally observed $A_{2}$ and $A_{4}$ coefficients of the angular distributions (see table 3 ), the expected polarization can be calculated except for the sign (see column six). The conclusions regarding the parity of the radiation and of the levels are indicated in the last columns.

At the 860 and $1097 \mathrm{keV}$ resonances all measurements are conclusive, thereby determining the parities of the $1.98,4.19,4.97,5.19$ and $9.34 \mathrm{MeV}$ levels. The radiation mixing ratio of the $0.78 \mathrm{MeV}$ gamma ray, observed at the $860 \mathrm{keV}$ resonance can have six different values, four values if $J=2$ is assigned to the $4.97 \mathrm{MeV}$ level, and two values if $J=3$ is assigned to this level. In all cases the parity of the radiation is the same. At the $1761 \mathrm{keV}$ resonance only the parity of the $4.43 \mathrm{MeV}$ level is determined beyond any doubt. The parities of the 7.18 and $10.22 \mathrm{MeV}$ levels remain uncertain; the favoured values are indicated. A better determination of the parity of the $7.18 \mathrm{MeV}$ level may be possible if the angular distribution of the $2.73 \mathrm{MeV}$ gamma ray can be measured more accurately. The parities of some transitions were determined at more than one resonance. All results are presented.

\section{Summary and Conclusions}

Before this investigation was undertaken, no spins or parities of levels at low excitation energies in ${ }^{36} \mathrm{Ar}$ had been determined. Suggestions about the spins of the 1.98 , 4.19 and $4.97 \mathrm{MeV}$ levels were made from angular distribution measurements in the ${ }^{35} \mathrm{Cl}(\mathrm{p}, \gamma)^{36} \mathrm{Ar}$ reaction as early as 1957 (ref. $\left.{ }^{37}\right)$ ) and 1960 (ref. $\left.{ }^{38}\right)$ ). Since then, numerous investigations have shown that the assumption of pure radiation of lowest multipolarity made at that time is by no means always true. 


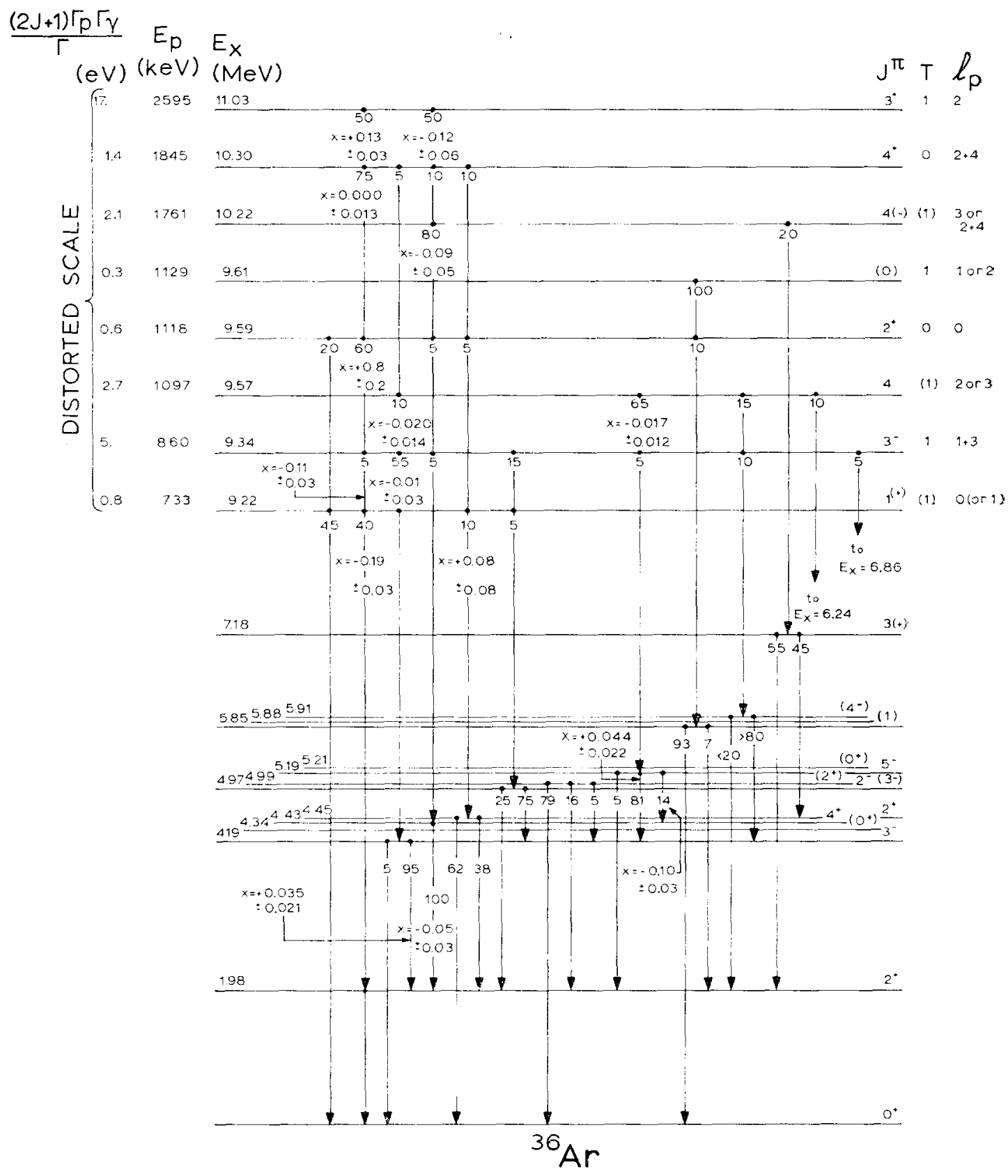

Fig. 13. Decay scheme of ${ }^{36} \mathrm{Ar}$. The energies, decay modes and resonance strengths are taken from ref. ${ }^{4}$ ); spins, parities, isospins, $l_{p}$ values and mixing ratios follow from the present investigation. The level energies up to $E_{x}=7.18 \mathrm{MeV}$ are taken from ref. 1 . The level scheme is drawn completely up to $5.91 \mathrm{MeV}$. 
If radiation mixtures are to be taken into account, the description of the angular correlations requires many parameters, which have to be determined experimentally. In the present investigation $\chi^{2}$ methods have been used to determine unique solutions for spin values and radiation mixing parameters.

Several levels in ${ }^{36} \mathrm{Ar}$ decay by multiple cascades. In these cases many triple correlation measurements can be performed, and generally they are needed to obtain definite conclusions. The methods of analysis should remain feasible, especially with the large amount of experimental data to be handled. As not much work has been done on multiple cascades up to now, the methods used in the present investigation are described in some detail. A summary of the theoretical formulation is included in this paper. Recently Harris, Hennecke and Watson ${ }^{39}$ ) have made different proposals for analysis, but no examples of applications were given.

The eight resonances considered were purposely chosen from 65 previously investigated ones ${ }^{4}$ ) because of their clear decay modes, large radiation anisotropies and for relevance to particular levels at low excitation energies. Not all measurements have given unique results; the resonances with isotropic angular distributions only seldom yield any conclusive evidence.

A summary of the experimental results is given in fig. 13. The spin assignments to 13 levels are unique; the uncertain assignments are given in parentheses. The spins given for the 4.97, 5.85, 5.91 and $9.61 \mathrm{MeV}$ levels are uncertain, though strongly favoured by decay properties and measurements of angular distributions or triple correlations. The tentative $2^{+}$assignment to the $4.99 \mathrm{MeV}$ level is derived from the decay properties only. The conclusion $J=0^{+}$, suggested for the 4.34 and $5.21 \mathrm{MeV}$ levels, is a good guess rather than an assignment.

Polarization measurements at the 860,1097 and $1761 \mathrm{keV}$ resonances determined the parities of the levels at $1.98,4.19,4.43,4.97,5.19$ and $9.34 \mathrm{MeV}$ (see fig. 13). The parities of the $4.45,9.59$ and $10.30 \mathrm{MeV}$ levels followed from a consideration of radiation strengths. The parity of the $11.03 \mathrm{MeV}$ resonance level was derived from the formation parameters.

The parity and isospin assignments to the $9.59 \mathrm{MeV}$ level are confirmed by the observation of alpha particles from the ${ }^{35} \mathrm{Cl}\left(\mathrm{p}, \alpha_{0}\right){ }^{32} \mathrm{~S}$ reaction by Bošnjakovič $\left.{ }^{28}\right)$. No alpha particles were observed at the other resonances investigated.

Isospin selection rules for E1 and M1 radiation appear to be effective in this selfconjugate nucleus. The 1118 and $1845 \mathrm{keV}$ resonances mainly decay through E2 radiation. In the decay of the $3^{-}$level at $4.19 \mathrm{MeV}$ an E3 ground-state transition competes with the $\mathrm{E} 1$ transition to the first excited state. The $5^{-}$level at $5.19 \mathrm{MeV}$ mainly decays through E2 radiation to the $4.19 \mathrm{MeV}$ level with weaker E1/M2 and E3 decay modes to the 4.43 and $1.98 \mathrm{MeV}$ levels. The isospin assignments to the resonances, derived from the observed strengths of dipole transitions, are also given in fig. 13. The $860 \mathrm{keV}$ resonance may be the isobaric analogue of the $2.47 \mathrm{MeV}$ level in ${ }^{36} \mathrm{Cl}$, for which van Middelkoop and Spilling ${ }^{25}$ ) recently found $J^{\pi}=3^{-}$from the ${ }^{35} \mathrm{Cl}(\mathrm{n}, \gamma){ }^{36} \mathrm{Cl}$ reaction. After correction for the Coulomb energy and the proton- 


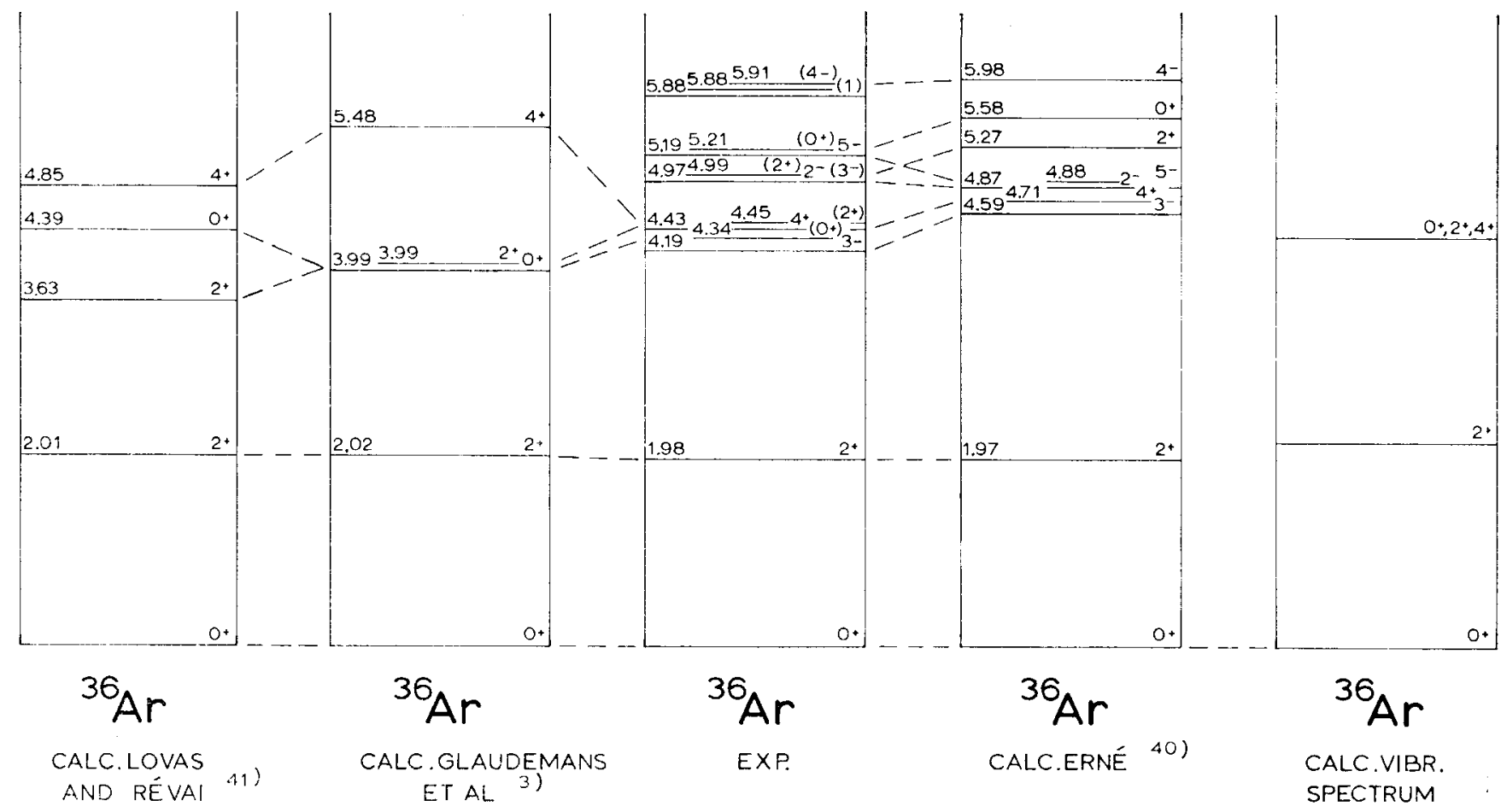

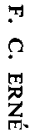

Fig. 14. Experimental and predicted level energies for ${ }^{36} \mathrm{Ar}$. The predicted level positions and their experimental equivalents are interconnected by dashed lines. 
neutron mass difference the level energies differ by about $200 \mathrm{keV}$.

Interpretation of the population parameters of the resonances in terms of formation through the lowest possible incoming proton angular momentum is not always possible. The formation of the $860 \mathrm{keV}$ resonance takes place via p-capture with $16 \pm 4 \% \mathrm{f}$-capture, the formation of the $1845 \mathrm{keV}$ resonance via d-capture with $4.0 \pm 1.3 \%$ g-capture. Other solutions with higher percentages of $\mathrm{f}$ - and g-capture, respectively, are not excluded in these cases.

The proton energies, the resonance strengths, $l_{\mathrm{p}}$ values and assigned isospins are also given in fig. 13. The decay modes of the resonances and the lower excited states involved in this investigation, are taken from ref. ${ }^{4}$ ). The values of the mixing ratios of several transitions are indicated.

The average strength of the Ml transitions from resonances that were established in the present investigation, exceeds that of the E1 transition by about a factor 20 . This substantially differs from the factor 3 that can be inferred from transitions in other nuclei in the s-d shell (see ref. ${ }^{29}$ )).

A number of shell-model calculations has been performed, describing even parity levels in ${ }^{36} \mathrm{Ar}$. Glaudemans, Wiechers and Brussaard ${ }^{3}$ ) considered a closed-shell ${ }^{28} \mathrm{Si}$ core with nucleons in the $2 \mathrm{~s}_{\frac{1}{2}}$ and $1 \mathrm{~d}_{\frac{3}{2}}$ shells. They calculated level energies for nuclei from ${ }^{29} \mathrm{Si}$ up to ${ }^{40} \mathrm{Ca}$ from 17 adjusted energy parameters. The results for ${ }^{36} \mathrm{Ar}$ are shown in fig. 14. Excitation energies in ${ }^{36} \mathrm{Ar}$ and ${ }^{36} \mathrm{Cl}$ were calculated by Lovas and Révai ${ }^{41}$ ) from similar assumptions. In their calculations Serber forces and a Yukawa potential were used; the difference between single-particle energies in the $2 \mathrm{~s}_{\frac{1}{2}}$ and $1 \mathrm{~d}_{\frac{3}{2}}$ shells was taken as an adjustable parameter. Their calculated level scheme is given in fig. 14. The experimental level scheme is also shown in fig. 14. The predicted level positions and their experimental equivalents are interconnected by dashed lines. The lowest $0^{+}$and $2^{+}$levels show good agreement. The energy of the $4^{+}$level is lower than the calculated one, especially in the calculation of Glaudemans et al. ${ }^{3}$ ). One may therefore expect appreciable mixing with configurations that result from excitation of one or more nucleons from the $1 \mathrm{~d}_{\frac{5}{2}}$ shell. Knowledge of the energies of $4^{+}$levels in ${ }^{38} \mathrm{Ar}$ would be relevant in this respect. The third $2^{+}$level at $4.99 \mathrm{MeV}$ was not predicted in either of the calculations. The next $2^{+}$level was calculated at $7.67 \mathrm{MeV}$ by Glaudemans et al. ${ }^{3}$ ).

The observed odd-parity levels can be interpreted as mainly arising from excitation of one nucleon from the $1 \mathrm{~d}_{\frac{3}{2}}$ shell to the $1 \mathrm{f}_{\frac{3}{2}}$ shell. A shell-model calculation has been performed by the author ${ }^{40}$ ), in which the level energies are calculated in the $j-j$ coupling scheme with methods proposed by Talmi and de-Shalit ${ }^{23}$ ). In this calculation the energies of 60 even- and odd-parity levels in nuclei from ${ }^{33} \mathrm{~S}$ to ${ }^{41} \mathrm{Ca}$ have been fitted to 14 energy parameters. The calculated energies are shown in fig. 14. The $2^{+}$level at $4.99 \mathrm{MeV}$ was interpreted as the seniority-4 level in the $1 \mathrm{~d}_{\frac{3}{2}}$ shell.

The deformation of the average field in ${ }^{36} \mathrm{Ar}$ is probably small. An indication for this can be obtained from the E2 transition probability of the $1.76 \mathrm{MeV}$ transition in the neighbouring nucleus $\mathrm{Cl}^{35}$ (see subsect. 2.4 .8 of this paper). This fits into the 
trend of E2 transition probabilities plotted by the Chalk River group ${ }^{32}$ ) for the s-d shell nuclei. The excitation energy of the lowest $2^{+}$level in ${ }^{36} \mathrm{Ar}(1.98 \mathrm{MeV})$ is slightly lower than the excitation energies of the $2^{+}$levels in ${ }^{32} \mathrm{~S},{ }^{34} \mathrm{~S}$ and ${ }^{38} \mathrm{Ar}(2.24,2.13$ and $2.17 \mathrm{MeV}$, respectively), which might indicate a small deformation of the average field. A number of highly deformed states may occur at high excitation energies.

The even-parity levels at low excitation energies in ${ }^{36} \mathrm{Ar}$ show the $0^{+}, 2^{+}, 0^{+} 2^{+} 4^{+}$ sequence that is characteristic for vibrational nuclei. The energies of the second $0^{+}$ and $2^{+}$and of the $4^{+}$level are found at about twice the excitation energy of the first $2^{+}$level. A vibrational spectrum is shown in fig. 14, for comparison.

From the proton decay at the $2595 \mathrm{keV}$ resonance the lowest two levels in ${ }^{35} \mathrm{Cl}$ are excited. The partial lifetimes of the $1.76 \mathrm{MeV}$ level in ${ }^{35} \mathrm{Cl}$ and the forbidden character of the proton decay to the $1.22 \mathrm{MeV}$ level in ${ }^{35} \mathrm{Cl}$ are consistent with configurations for these levels consisting of one nucleon excited from the $2 \mathrm{~s}$ orbit into the $1 \mathrm{~d}$ orbit.

The authors thanks Professor P. M. Endt for his excellent guidance in all stages of the investigation. The spiritual methods of experimenting and analysing by Professor P. B. Smith were greatly stimulating. The frequent advice of Dr. C. van der Leun were gratefully accepted.

Also Dr. P. J. Brussaard and Dr. D. M. Sheppard kindly criticized the manuscript. Discussions with my colleagues Dr. A. Heyligers, Dr. P. W. M. Glaudemans, P. H. Vuister, Dr. P. J. M. Smulders and Dr. J. Kuperus contributed much to the final result.

The help of W.A.M. Veltman during long runs is appreciated. Dr. H. A. Cysouw contributed by chemically converting isotopic material. The experiments were greatly facilitated by the technical staff of the Van de Graaff accelerator.

This investigation was partly supported by the joint program of the "Stichting voor Fundamenteel Onderzoek der Materie" and the "Nederlandse Organisatie voor Zuiver Wetenschappelijk Onderzoek."

\section{References}

1) R. G. Allas, L. Meyer-Schützmeister and D. von Ehrenstein, Nuclear Physics 61 (1965) 289

2) F. C. Erné and C. van der Leun, Nuclear Physics 52 (1964) 515

3) P. W. M. Glaudemans, G. Wiechers and P. J. Brussaard, Nuclear Physics 56 (1964) 529, 548

4) F. C. Erné and P. M. Endt, Nuclear Physics 71 (1965) 593

5) A. Heyligers, Thesis, Utrecht (1964)

6) P. W. M. Glaudemans and P. M. Endt, Nuclear Physics 42 (1963) 367

7) J. Kuperus, P. W. M. Glaudemans and P. M. Endt, Physica 29 (1963) 327

8) A. E. Litherland and A. J. Ferguson, Can. J. Phys. 39 (1961) 788

9) P. B. Smith, in Nuclear reactions, Vol. II, ed. by P. M. Endt and P. B. Smith (North-Holland Publ. Co., Amsterdam, 1962)

10) C. Broude and H. E. Gove, Ann. of Phys. 23 (1963) 71

11) R. Nordhagen, Nuclear Physics 44 (1963) 130; R. Nordhagen and A. Tveter, Nuclear Physics 56 (1964) 337, 63 (1965) 529

12) S. Devons and L. J. B. Goldfarb, Handbuch der Physik, Vol.42, ed. by S. Flügge (Springer, Berlin, 1957) p. 362 
13) A. J. Ferguson, Angular correlation methods (North-Holland Publ. Co., Amsterdam, 1965)

14) H. A. Van Rinsvelt and P. B. Smith, Physica 30 (1964) 59

15) P. J. M. Smulders and A. H. E. van Hengel, unpublished, Utrecht (1965)

16) L. C. Biedenharn, Nuclear spectroscopy, part B, ed. by. F. Ajzenberg-Selove (Academic Press, New York, 1960) chapt. VC

17) E. Jahnke, F. Emde and F. Lösch, Tables of higher functions (Teubner, Stuttgart, 1960)

18) A. J. Ferguson and A. R. Rutledge, Atomic Energy of Canada Report AECL-420 (1957) revised and reprinted (1962)

19) H. E. Gove, in Nuclear reactions, Vol. I, ed. by P. M. Endt and M. Demeur (North-Holland Publ. Co., Amsterdam, 1959)

20) G. Kaye, E. J. C. Read and J. C. Willmott, Tables of Coefficients for the Analysis of Triple Correlations from Aligned Nuclei, Chadwick Physics Laboratory, University of Liverpool, England (1963)

21) G. J. Nijgh, A. H. Wapstra and R. van Lieshout, Nuclear spectroscopy tables (North-Holland Publ. Co., Amsterdam, 1959)

22) P. B. Smith, Can. J. Phys. 42 (1964) 1101

23) A. de-Shalit and I. Talmi, Nuclear shell theory (Academic Press, New York, 1963)

24) R. Nordhagen, Nucl. Instr. 26 (1964) 353

25) G. van Middelkoop and P. Spilling, Nuclear Physics 77 (1966) 267

26) A. M. Hoogenboom, E. Kashy and W. W. Buechner, Phys. Rev. 128 (1962) 305

27) B. L. Robinson, Phys. Rev. 140 (1965) B1529

28) B. Bošnjaković, Utrecht, private communication

29) C. van der Leun, in Proc. Lawrence, Kansas Symposium on the Structure of Low-Medium Mass Nuclei (1964)

30) N. Hazewindus, Thesis, Delft (1964)

31) P. M. Endt and C. van der Leun, Nuclear Physics 34 (1962) 1

32) A. E. Litherland, in Nuclear structure and electromagnetic interactions, Scottish Universities Summer School, ed. by N. McDonald (Oliver and Boyd, Edinburgh, 1964)

33) P. M. Endt and A. Heyligers, Physica 26 (1960) 230

34) H. P. Leenhouts and P. M. Endt, Physica, submitted

35) M. Suffert, P. M. Endt and A. M. Hoogenboom, Physica 25 (1959) 659

36) H. Van Rinsvelt and P. M. Endt, Phys. Lett. 9 (1964) 266

37) R. Berenbaum and J. H. Matthews, Proc. Phys. Soc. A70 (1957) 445

38) J. C. Lisle and P. F. D. Shaw, Proc. Phys. Soc. 76 (1960) 929

39) G. I. Harris, H. J. Hennecke and D. D. Watson, Phys. Rev. 139 (1965) B1113

40) F. C. Erné, to be published

41) I. Lovas and J. Révai, Nuclear Physics 59 (1965) 364

42) F. C. Erné, Phys. Lett. 19 (1965) 590

43) E. C. Booth and K. A. Wright, Nuclear Physics 35 (1962) 472 